\title{
Photoinduced Decarboxylative Radical Coupling Reaction of Multiply Oxygenated Structures by Catalysis of Pt-Doped $\mathrm{TiO}_{2}$
}

Daiki Kuwana, Yuma Komori, Masanori Nagatomo and Masayuki Inoue*

Graduate School of Pharmaceutical Sciences

The University of Tokyo, Hongo, Bunkyo-ku, Tokyo 113-0033, Japan

Fax: $(+81) 3-5841-0568$

E-mail:inoue@mol.f.u-tokyo.ac.jp

Supporting Information

73 pages

Contents:

page

1. Experimental procedures

2. Hydrogen transfer experiments

3. MPT-623 recycling experiments

4. Experiments on $1 \mathrm{mmol}$ scale

5. Investigation of the light source

6. Attempted Ir-catalyzed dimerization and radical addition

7. References

8. ${ }^{1} \mathrm{H}$ and ${ }^{13} \mathrm{C}\left\{{ }^{1} \mathrm{H}\right\}$ NMR spectra 


\section{Experimental procedures}

General method: All reactions sensitive to air or moisture were carried out under argon atmosphere in dry solvents unless otherwise noted. $\mathrm{CH}_{2} \mathrm{Cl}_{2}$ and DMF were purified by Glass Contour solvent dispensing system (Nikko Hansen \& Co., Ltd.). Aeroxide P25 $\mathrm{TiO}_{2}$, consisting of a 4:1 mixture of anatase and rutile phases (particle size $\sim 21 \mathrm{~nm}$, surface area 50 $\mathrm{m}^{2} / \mathrm{g}$ ), was obtained from Evonik Industries. Pt-doped $\mathrm{TiO}_{2}$ (MPT-623), consisting of 97.7 $\mathrm{wt} \% \mathrm{TiO}_{2}$ and $0.075-0.125 \mathrm{wt} \% \mathrm{Pt}$ (particle size $\sim 18 \mathrm{~nm}$, surface area $58 \mathrm{~m}^{2} / \mathrm{g}$ ), was obtained from Ishihara Sangyo Kaisha Ltd. All other reagents were used as supplied. Sonication was conducted with a US-3 ultrasonic cleaner (SND Co., Ltd, $38 \mathrm{kHz}$ ). Photoreactions were carried out in borosilicate glass test tubes or round bottom flasks. A tube or a flask was placed at $0 \mathrm{~cm}$ from a $40 \mathrm{~W}$ violet LED (PR160L-390 nm, 10,600 lx, Kessil). The reaction system was externally cooled with a circulator (C:NET Co., Ltd., $2050 \mathrm{rpm}$ ). Analytical thin-layer chromatography (TLC) was performed using Merck Silica gel $60 \mathrm{~F}_{254}$ pre-coated plates $(0.25$ $\mathrm{mm}$ ). Preparative thin-layer chromatography (PTLC) was performed using Merck Silica gel $60 \mathrm{~F}_{254}$ pre-coated plates $(0.5 \mathrm{~mm})$. Flash column chromatography was performed using 40 $50 \mu \mathrm{m}$ Silica Gel 60N (Kanto Chemical Co., Inc.). Automated flash chromatography was conducted with a Yamazen Smart Flash EPCLC-AI-580S system using the prepacked silica gel columns. Unless otherwise noted, Silica Gel 60N was used for the purification. A $33 \mathrm{~mm}$ diameter syringe filter with a $0.45 \mu \mathrm{m}$ pore size hydrophilic polyethersulfone (PES) membrane (Merck Millipore Ltd.) was used for membrane filtration unless otherwise noted. Melting points were measured on Yanaco MP-J3 micro melting point apparatus, and were uncorrected. Optical rotations were measured on JASCO P-2200 polarimeter at room temperature using sodium D line. Infrared (IR) spectra were recorded on JASCO FT/IR-4100 spectrometer as a thin film on $\mathrm{KBr} . \quad{ }^{1} \mathrm{H}$ and ${ }^{13} \mathrm{C}\left\{{ }^{1} \mathrm{H}\right\}$ NMR spectra were recorded on JNM-ECS-400, JNM-ECX500, or JNM-ECZ-500 spectrometer. Chemical shifts were denoted in ppm on the $\delta$ scale relative to residual solvent peaks as an internal standard: $\mathrm{CHCl}_{3}\left(\delta 7.26\right.$ for $\left.{ }^{1} \mathrm{H} \mathrm{NMR}\right), \mathrm{CDCl}_{3}$ ( $\delta 77.0$ for ${ }^{13} \mathrm{C}\left\{{ }^{1} \mathrm{H}\right\}$ NMR), $\mathrm{C}_{6} \mathrm{D}_{5} \mathrm{H}$ ( $\delta 7.16$ for ${ }^{1} \mathrm{H}$ NMR), and $\mathrm{C}_{6} \mathrm{D}_{6}\left(\delta 128.0\right.$ for $\left.{ }^{13} \mathrm{C}\left\{{ }^{1} \mathrm{H}\right\} \mathrm{NMR}\right)$. Signal patterns are indicated as s, singlet; $d$, doublet; $t$, triplet; $\mathrm{m}$, multiplet; br, broaden peak. High-resolution mass spectra were measured on JEOL JMS-T100LP (ESI-TOF) or BRUKER DALTONICS microTOF II (ESI-TOF). 
<smiles>O=C(O)C(c1ccccc1)c1ccccc1</smiles>

1a

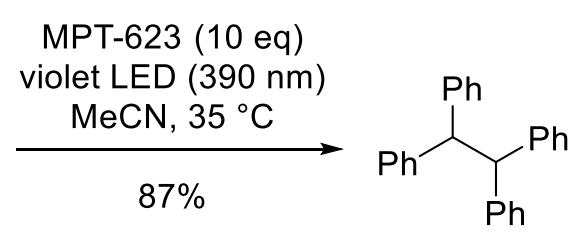

$3 a$

Dimer 3a [CAS: 632-50-8]. Argon was bubbled through a suspension of carboxylic acid 1a (42.4 mg, $200 \mu \mathrm{mol})$ and MPT-623 (160 mg, $2.00 \mathrm{mmol})$ in $\mathrm{MeCN}(2.0 \mathrm{~mL})$ at $23{ }^{\circ} \mathrm{C}$ for 20 min. The resultant mixture was sonicated for $3 \mathrm{~min}$, stirred, and then irradiated with a violet LED at $35^{\circ} \mathrm{C}$. After being irradiated with stirring at $35^{\circ} \mathrm{C}$ for $3 \mathrm{~h}$, the resultant mixture was filtered and concentrated. The residue was purified by automated column chromatography using the prepacked silica gel $(16 \mathrm{~g}$, hexane/EtOAc $=1 / 0$ to $83 / 17)$ to afford dimer $\mathbf{3 a}(29.0 \mathrm{mg}$, $86.7 \mu \mathrm{mol})$ in $87 \%$ yield. The analytical data of 3a was identical to those reported previously. ${ }^{\mathrm{S} 1}$

${ }^{1} \mathrm{H}$ NMR (400MHz, $\left.\mathrm{CDCl}_{3}\right): \delta$ 7.18-7.16 (m, 8H), 7.13-7.09 (m, 8H), 7.04-6.99 (m, 4H), 4.77 (s, 2H).
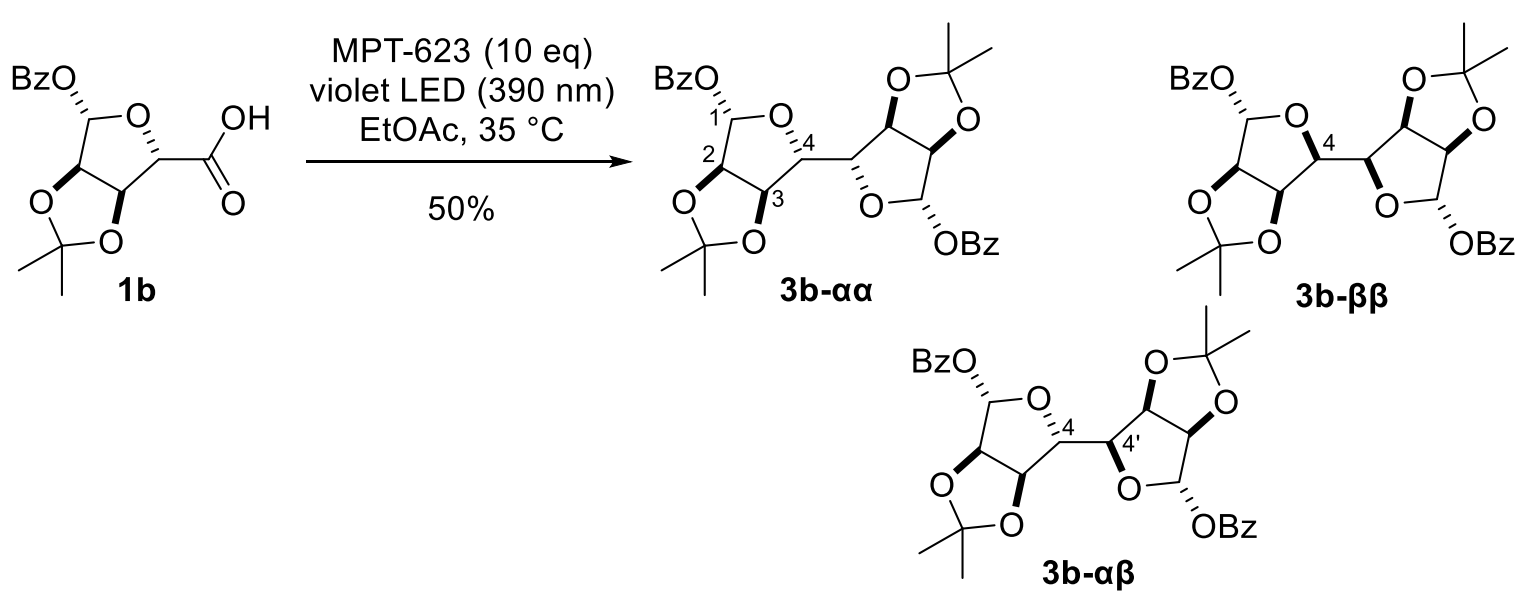

General procedure A: Dimer 3b. Argon was bubbled through a suspension of carboxylic acid 1b [CAS: $2043350-11-2]^{\mathrm{S} 2}(30.8 \mathrm{mg}, 100 \mu \mathrm{mol})$ and MPT-623 (80 mg, $\left.1.00 \mathrm{mmol}\right)$ in EtOAc $(1.0 \mathrm{~mL})$ at $23{ }^{\circ} \mathrm{C}$ for $20 \mathrm{~min}$. The resultant mixture was sonicated for $3 \mathrm{~min}$, stirred, and then irradiated with a violet LED at $35^{\circ} \mathrm{C}$. After being irradiated with stirring at $35^{\circ} \mathrm{C}$ for $16 \mathrm{~h}$, the resultant mixture was filtered and concentrated. The residue was purified by automated column chromatography using the prepacked silica gel $(16 \mathrm{~g}$, hexane/EtOAc $=84 / 16$ to $63 / 37$ ) to afford a 30:60:10 diastereomeric mixture of $3 \mathbf{b}-\boldsymbol{\alpha} \boldsymbol{\alpha}$ [CAS: 2043350-42-9], $\mathbf{3 b} \mathbf{b} \boldsymbol{\alpha} \boldsymbol{\beta}$

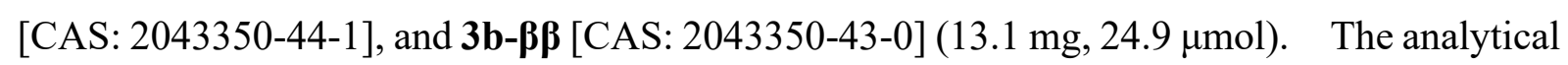
data of $\mathbf{3 b}$ was identical to those reported previously. ${ }^{\mathrm{S} 2}$

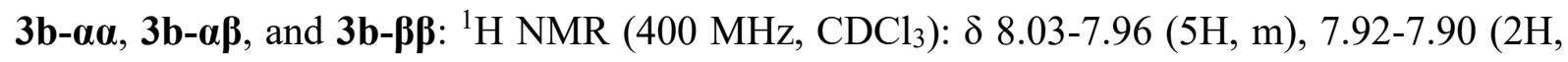
m), 7.60-7.56 (3H, m), 7.53-7.32 (9H, m), $6.53(2 \mathrm{H} \mathrm{x3/10,} \mathrm{s),} 6.51(1 \mathrm{H} \mathrm{x6/10,} \mathrm{s),} 6.47(1 \mathrm{H}$ 
x1/10, s), $6.46(1 \mathrm{H} \mathrm{x6/10,} \mathrm{s),} 5.09(1 \mathrm{H}, \mathrm{d}, J=6.0 \mathrm{~Hz}), 4.90-4.81(6 \mathrm{H}, \mathrm{m}), 4.75(1 \mathrm{H} \mathrm{x6/10,} \mathrm{d,} J$ $=8.2 \mathrm{~Hz}, \mathrm{H} 4$ '), $4.46(2 \mathrm{H} \mathrm{x1} / 10, \mathrm{~s}, \mathrm{H} 4), 4.39(2 \mathrm{H} \mathrm{x} 3 / 10, \mathrm{~s}, \mathrm{H} 4), 4.31(1 \mathrm{H} \mathrm{x6} / 10, \mathrm{dd}, J=8.2,3.2$ Hz, H4), 1.56 (6H, s), $1.54(4 \mathrm{H}, \mathrm{s}), 1.53$ (2H, s), $1.38(2 \mathrm{H}, \mathrm{s}), 1.36(5 \mathrm{H}, \mathrm{s}), 1.34(6 \mathrm{H}, \mathrm{s})$.
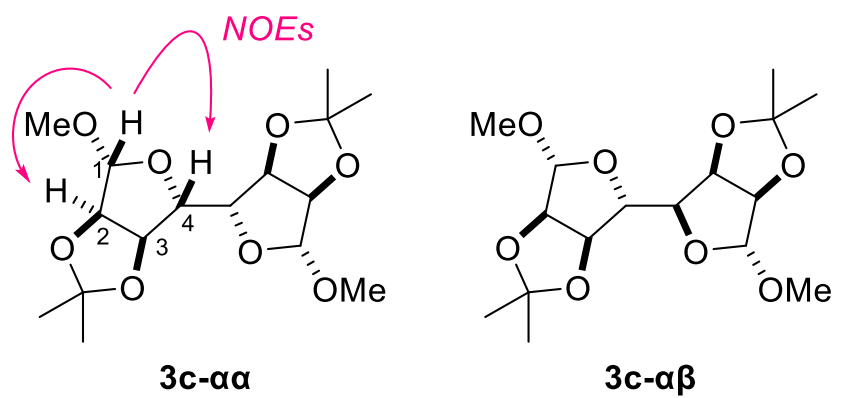

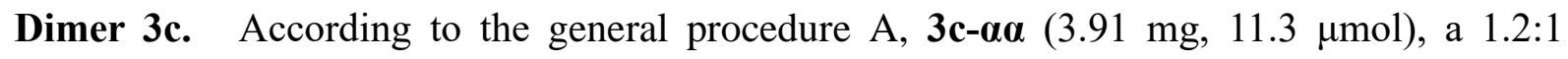
diastereomeric mixture of $\mathbf{3 c - \alpha \boldsymbol { \alpha }}$ and $\mathbf{3 c - \alpha} \boldsymbol{\beta}$ (3.61 mg, $10.4 \mu \mathrm{mol})$, and $\mathbf{3 c - \alpha} \boldsymbol{\beta}$ (4.96 mg, 14.3 $\mu \mathrm{mol})$ were synthesized from carboxylic acid 1c [CAS: 54622-95-6] ${ }^{\mathrm{S} 3}$ (22.0 mg, $\left.101 \mu \mathrm{mol}\right)$ by using MPT-623 (80.9 mg, $1.01 \mathrm{mmol})$ in EtOAc $(1.0 \mathrm{~mL})$. After being irradiated with stirring at $35^{\circ} \mathrm{C}$ for $16 \mathrm{~h}$, the resultant mixture was filtered and concentrated. The residue was purified by automated flash column chromatography using the prepacked silica gel ( $4 \mathrm{~g}$, hexane/EtOAc $=81 / 19$ to $60 / 40$ ). The combined yield of two diastereomers $3 \mathbf{c}$ was $73 \%$, and the $\alpha \alpha / \alpha \beta$ ratio

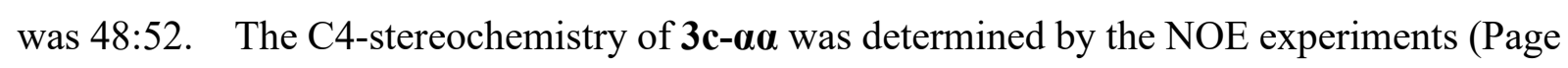
S27).

3c- $\alpha \alpha$ : colorless oil. $[\alpha]_{\mathrm{D}}{ }^{24}-53.8$ (c 2.2, $\mathrm{CHCl}_{3}$ ). IR (film): 2986, 2935, 2834, 1447, 1377, 1271, 1242, 1210, 1161, 1094, $1052 \mathrm{~cm}^{-1} .{ }^{1} \mathrm{H}$ NMR (400 MHz, $\left.\mathrm{CDCl}_{3}\right): \delta 5.00(2 \mathrm{H}, \mathrm{s}, \mathrm{H} 1), 4.60(2 \mathrm{H}$, $\mathrm{d}, J=6.0 \mathrm{~Hz}, \mathrm{H} 2), 4.58(2 \mathrm{H}, \mathrm{d}, J=6.0 \mathrm{~Hz}, \mathrm{H} 3), 4.04(2 \mathrm{H}, \mathrm{s}, \mathrm{H} 4), 3.40\left(6 \mathrm{H}, \mathrm{s}, \mathrm{OCH}_{3}\right), 1.47(6 \mathrm{H}$, s, $\mathrm{CH}_{3}$ of acetonide), $1.30\left(6 \mathrm{H}, \mathrm{s}, \mathrm{CH}_{3}\right.$ of acetonide). ${ }^{13} \mathrm{C}\left\{{ }^{1} \mathrm{H}\right\} \mathrm{NMR}\left(100 \mathrm{MHz}, \mathrm{CDCl}_{3}\right): \delta 112.5$ (2C), 108.9 (2C), 88.0 (2C), 85.1 (2C), 81.3 (2C), 54.7 (2C), 26.3 (2C), 24.7 (2C). HRMS (ESITOF) m/z: [M+Na $]^{+}$Calcd for $\mathrm{C}_{16} \mathrm{H}_{26} \mathrm{O}_{8} \mathrm{Na}$ 369.1520; Found 369.1531.

3c- $\boldsymbol{\alpha \beta}$ : colorless solid. m.p. $95-100{ }^{\circ} \mathrm{C}$. $[\alpha]_{\mathrm{D}}{ }^{24}-71.4$ (c 2.2, $\left.\mathrm{CHCl}_{3}\right)$. IR (film): 2987, 2939, 2834 , 1456, 1375, 1271, 1210, 1163, 1100, $1032 \mathrm{~cm}^{-1} .{ }^{1} \mathrm{H}$ NMR (400 MHz, $\left.\mathrm{CDCl}_{3}\right): \delta 5.00(1 \mathrm{H}, \mathrm{s})$, $4.89(1 \mathrm{H}, \mathrm{s}), 4.86(1 \mathrm{H}, \mathrm{d}, J=6.0 \mathrm{~Hz}), 4.72(1 \mathrm{H}, \mathrm{dd}, J=6.0,3.7 \mathrm{~Hz}), 4.62(1 \mathrm{H}, \mathrm{d}, J=6.0 \mathrm{~Hz})$, $4.57(1 \mathrm{H}, \mathrm{d}, J=6.0 \mathrm{~Hz}), 4.47(1 \mathrm{H}, \mathrm{d}, J=10.1 \mathrm{~Hz}), 3.87(1 \mathrm{H}, \mathrm{dd}, J=10.1,3.2 \mathrm{~Hz}), 3.36(3 \mathrm{H}$, s), $3.31(3 \mathrm{H}, \mathrm{s}), 1.49(6 \mathrm{H}, \mathrm{s}), 1.33(3 \mathrm{H}, \mathrm{s}), 1.32(3 \mathrm{H}, \mathrm{s}) .{ }^{13} \mathrm{C}\left\{{ }^{1} \mathrm{H}\right\}$ NMR $\left(100 \mathrm{MHz}, \mathrm{CDCl}_{3}\right): \delta$ 112.8, 112.5, 109.6, 107.6, 85.4, 85.2, 84.2, 82.7, 79.9, 79.3, 55.3, 54.7, 26.5, 25.9, 25.0, 24.7. HRMS (ESI-TOF) m/z: [M+Na] ${ }^{+}$Calcd for $\mathrm{C}_{16} \mathrm{H}_{26} \mathrm{O}_{8} \mathrm{Na} 369.1520$; Found 369.1535. 


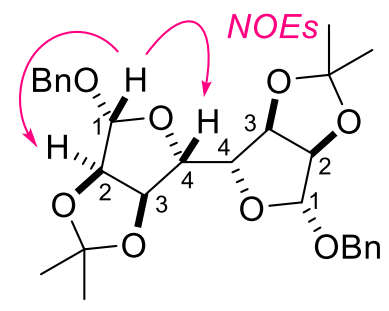

$3 d-\alpha \alpha$

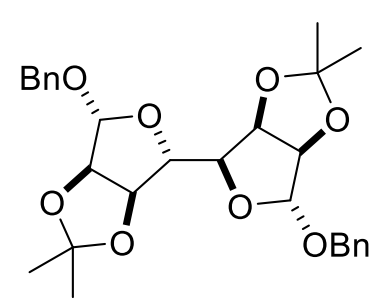

$3 d-\alpha \beta$

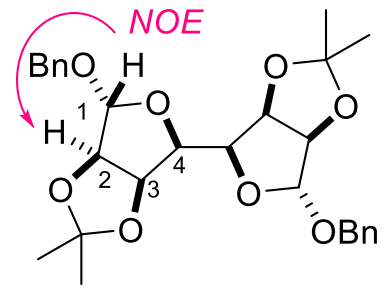

$3 d-\beta \beta$

Dimer 3d. According to the general procedure A, 3d- $\alpha \boldsymbol{\alpha}(5.00 \mathrm{mg}, 10.0 \mu \mathrm{mol}), \mathbf{3 d}-\boldsymbol{\alpha} \boldsymbol{\beta}(5.10$ $\mathrm{mg}, 10.2 \mu \mathrm{mol})$, and $\mathbf{3 d - \beta} \boldsymbol{\beta}(0.80 \mathrm{mg}, 1.6 \mu \mathrm{mol})$ were synthesized from carboxylic acid $\mathbf{1 d}$ [CAS: $68673-83-6]^{\mathrm{S} 4}(29.4 \mathrm{mg}, 100 \mu \mathrm{mol})$ by using MPT-623 $(80.0 \mathrm{mg}, 1.00 \mathrm{mmol})$ in EtOAc $(1.0 \mathrm{~mL})$. After being irradiated with stirring at $35{ }^{\circ} \mathrm{C}$ for $16 \mathrm{~h}$, the resultant mixture was filtered and concentrated. The residue was purified by PTLC $(20 \mathrm{~cm}$ x $10 \mathrm{~cm}$, hexane/EtOAc $=10 / 1$ ). The combined yield of three diastereomers $3 \mathbf{d}$ was $43 \%$, and the $\alpha \alpha / \alpha \beta / \beta \beta$ ratio was 46:47:7. The C4-stereochemistry of $\mathbf{3 d - \alpha \alpha}$ was determined by the NOE experiment (Page $\mathrm{S} 30)$.

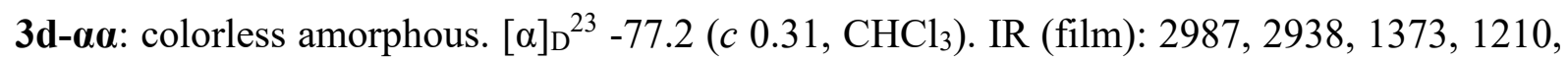
1079, 1001, $971 \mathrm{~cm}^{-1} .{ }^{1} \mathrm{H}$ NMR (400 MHz, $\left.\mathrm{CDCl}_{3}\right): \delta$ 7.42-7.26 (10H, m, aromatic), $5.24(2 \mathrm{H}$, s, H1), $4.95(2 \mathrm{H}, \mathrm{d}, J=11.3 \mathrm{~Hz}, \mathrm{OCHaHbPh}), 4.71(2 \mathrm{H}, \mathrm{d}, J=5.9 \mathrm{~Hz}, \mathrm{H} 2), 4.68$ (2H, d, $J=$ $5.9 \mathrm{~Hz}, \mathrm{H} 3), 4.55(2 \mathrm{H}, \mathrm{d}, J=11.3 \mathrm{~Hz}, \mathrm{OCHaHbPh}), 4.24(2 \mathrm{H}, \mathrm{s}, \mathrm{H} 4), 1.47\left(6 \mathrm{H}, \mathrm{s}, \mathrm{CH}_{3}\right.$ of acetonide), $1.31\left(6 \mathrm{H}, \mathrm{s}, \mathrm{CH}\right.$ of acetonide). ${ }^{13} \mathrm{C}\left\{{ }^{1} \mathrm{H}\right\} \mathrm{NMR}\left(100 \mathrm{MHz}, \mathrm{CDCl}_{3}\right): \delta 137.2(2 \mathrm{C})$, 128.6 (4C), 128.4 (4C), 127.9 (2C), 112.6 (2C), 106.6 (2C), 88.2 (2C), $85.4(2 \mathrm{C}), 81.4(2 \mathrm{C})$, 68.8 (2C), 26.4 (2C), 24.9 (2C). HRMS (ESI-TOF) m/z: [M+Na] ${ }^{+}$Calcd for $\mathrm{C}_{28} \mathrm{H}_{34} \mathrm{O}_{8} \mathrm{Na}$ 521.2146; Found 521.2142.

3d- $\alpha \boldsymbol{\beta}$ : colorless solid. m.p. $99-101{ }^{\circ} \mathrm{C}$. $[\alpha]_{\mathrm{D}}{ }^{24}-92.9$ (c 0.39, $\left.\mathrm{CHCl}_{3}\right)$. IR (film): 2986, 2938, 1373, 1209, 1088, $1036 \mathrm{~cm}^{-1} .{ }^{1} \mathrm{H}$ NMR (400 MHz, $\left.\mathrm{CDCl}_{3}\right): \delta 7.39-7.26(10 \mathrm{H}, \mathrm{m}), 5.19(1 \mathrm{H}, \mathrm{s})$, $5.11(1 \mathrm{H}, \mathrm{s}), 4.73(1 \mathrm{H}, \mathrm{d}, J=11.8 \mathrm{~Hz}), 4.54(1 \mathrm{H}, \mathrm{d}, J=11.8 \mathrm{~Hz}), 4.67-4.51(6 \mathrm{H}, \mathrm{m}), 4.48(1 \mathrm{H}$, $\mathrm{d}, J=10.4 \mathrm{~Hz}), 3.96(1 \mathrm{H}, \mathrm{dd}, J=10.4,3.2 \mathrm{~Hz}), 1.49(6 \mathrm{H}, \mathrm{s}), 1.34(3 \mathrm{H}, \mathrm{s}), 1.32(3 \mathrm{H}, \mathrm{s}) .{ }^{13} \mathrm{C}\left\{{ }^{1} \mathrm{H}\right\}$ NMR (100 MHz, $\mathrm{CDCl}_{3}$ ): $\delta 137.8,137.3,128.5$ (2C), $128.4(2 \mathrm{C}), 127.8(3 \mathrm{C}), 127.6(3 \mathrm{C}), 112.8$, 112.4 , 107.9, 106.3, 85.5, 85.3, 84.3, 82.5, 79.8, 79.3, 69.6, 69.5, 26.4, 26.0, 25.0, 24.7. HRMS (ESI-TOF) $\mathrm{m} / \mathrm{z}:[\mathrm{M}+\mathrm{Na}]^{+}$Calcd for $\mathrm{C}_{28} \mathrm{H}_{34} \mathrm{O}_{8} \mathrm{Na} 521.2146$; Found 521.2141.

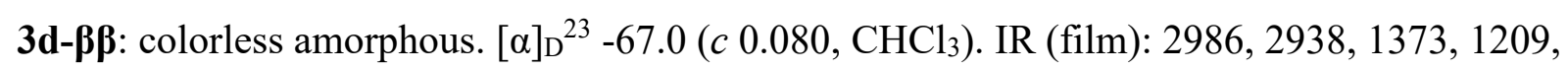
1114, 1077, $1007 \mathrm{~cm}^{-1} .{ }^{1} \mathrm{H}$ NMR (400 MHz, $\left.\mathrm{CDCl}_{3}\right): \delta$ 7.40-7.26 (10H, m, aromatic), $5.20(2 \mathrm{H}$, s, H1), 4.94 (2H, br d, $J=5.9 \mathrm{~Hz}, \mathrm{H} 3), 4.80(2 \mathrm{H}, \mathrm{d}, J=11.8 \mathrm{~Hz}, \mathrm{OCHaHbPh}), 4.67$ (2H, d, $J=$ $5.9 \mathrm{~Hz}, \mathrm{H} 2), 4.54(2 \mathrm{H}, \mathrm{d}, J=11.8 \mathrm{~Hz}, \mathrm{OCHaHbPh}), 4.28(2 \mathrm{H}, \mathrm{br} \mathrm{s}, \mathrm{H} 4), 1.44\left(6 \mathrm{H}, \mathrm{s}, \mathrm{CH}_{3}\right.$ of acetonide), $1.31\left(6 \mathrm{H}, \mathrm{s}, \mathrm{CH}_{3}\right.$ of acetonide). ${ }^{13} \mathrm{C}\left\{{ }^{1} \mathrm{H}\right\} \mathrm{NMR}\left(100 \mathrm{MHz}, \mathrm{CDCl}_{3}\right): \delta 137.4(2 \mathrm{C})$, 128.4 (4C), 128.1 (4C), 127.8 (2C), 112.4 (2C), $105.6(2 \mathrm{C}), 84.9$ (2C), 79.93 (2C), 79.85 (2C), 69.0 (2C), 26.1 (2C), 24.8 (2C). HRMS (ESI-TOF) m/z: [M+Na] ${ }^{+}$Calcd for $\mathrm{C}_{28} \mathrm{H}_{34} \mathrm{O}_{8} \mathrm{Na}$ 521.2146; Found 521.2154. 


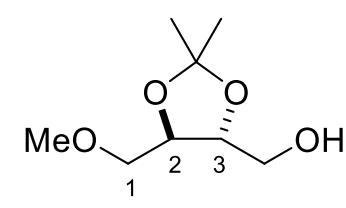

S1

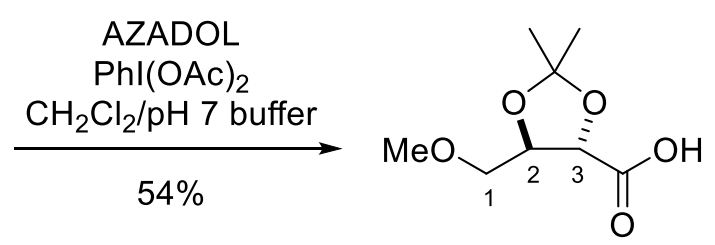

$1 e$

Carboxylic Acid 1e. 2-Hydroxy-2-azaadamantane (AZADOL, $10.7 \mathrm{mg}, 70.0 \mu \mathrm{mol}$ ) and $\mathrm{PhI}(\mathrm{OAc})_{2}(1.35 \mathrm{~g}, 420 \mathrm{mmol})$ were added to a solution of alcohol S1 [CAS: 331812-65-8] ${ }^{\mathrm{S} 5}$ (247 mg, $1.40 \mathrm{mmol})$ in $\mathrm{CH}_{2} \mathrm{Cl}_{2}(1.4 \mathrm{~mL})$ and $\mathrm{pH} 7$ phosphate buffer $(1.4 \mathrm{~mL})$ at $21^{\circ} \mathrm{C}$. After the reaction mixture was stirred at $21^{\circ} \mathrm{C}$ for $1 \mathrm{~h}$, saturated aqueous $\mathrm{Na}_{2} \mathrm{~S}_{2} \mathrm{O}_{3}(3 \mathrm{~mL})$ was added. The resultant solution was extracted with $\mathrm{CH}_{2} \mathrm{Cl}_{2}(3 \mathrm{~mL} \times 3)$. $1 \mathrm{M}$ aqueous $\mathrm{HCl}$ solution (1 $\mathrm{mL}$ ) was added to the aqueous layer and the resultant solution was extracted with $\mathrm{CHCl}_{3}(5 \mathrm{~mL}$ $\mathrm{x} 2$ ). The combined organic layers were dried over $\mathrm{Na}_{2} \mathrm{SO}_{4}$, filtered, and concentrated with $\mathrm{Et}_{3} \mathrm{~N}(1 \mathrm{~mL})$. The residue was purified by flash chromatography on silica gel $(3 \mathrm{~g}$, hexane/EtOAc $=3 / 1$ to $1 / 3)$ to afford carboxylic acid 1 e $(154 \mathrm{mg}, 810 \mu \mathrm{mol})$ in $54 \%$ yield: colorless oil. $[\alpha]_{\mathrm{D}}{ }^{20}+3.37$ (c 1.05, $\mathrm{CHCl}_{3}$ ). IR (film): 2991, 2934, 1740, 1380, 1209, 1097, 852 $\mathrm{cm}^{-1} .{ }^{1} \mathrm{H}$ NMR $\left(400 \mathrm{MHz}, \mathrm{CDCl}_{3}\right): \delta 4.39(1 \mathrm{H}, \mathrm{d}, J=7.8 \mathrm{~Hz}, \mathrm{H} 3), 4.33(1 \mathrm{H}, \mathrm{m}, \mathrm{H} 2), 3.72(1 \mathrm{H}$, $\mathrm{dd}, J=10.5,3.6 \mathrm{~Hz}, \mathrm{H} 1 \mathrm{a}), 3.66(1 \mathrm{H}, \mathrm{dd}, J=10.5,5.5 \mathrm{~Hz}, \mathrm{H} 1 \mathrm{~b}), 3.47\left(3 \mathrm{H}, \mathrm{s}, \mathrm{OCH}_{3}\right), 1.51(3 \mathrm{H}$, s, $\mathrm{CH}_{3}$ of acetonide), $1.47\left(3 \mathrm{H}, \mathrm{s}, \mathrm{CH}_{3}\right.$ of acetonide). ${ }^{13} \mathrm{C}\left\{{ }^{1} \mathrm{H}\right\} \mathrm{NMR}\left(100 \mathrm{MHz}, \mathrm{CDCl}_{3}\right): \delta 175.0$, 111.9, 77.8, 74.9, 72.2, 59.5, 26.7, 25.4. HRMS (ESI-TOF) m/z: [M-H]- Calcd for $\mathrm{C}_{8} \mathrm{H}_{13} \mathrm{O}_{5}$ 189.0768; Found 189.0771.
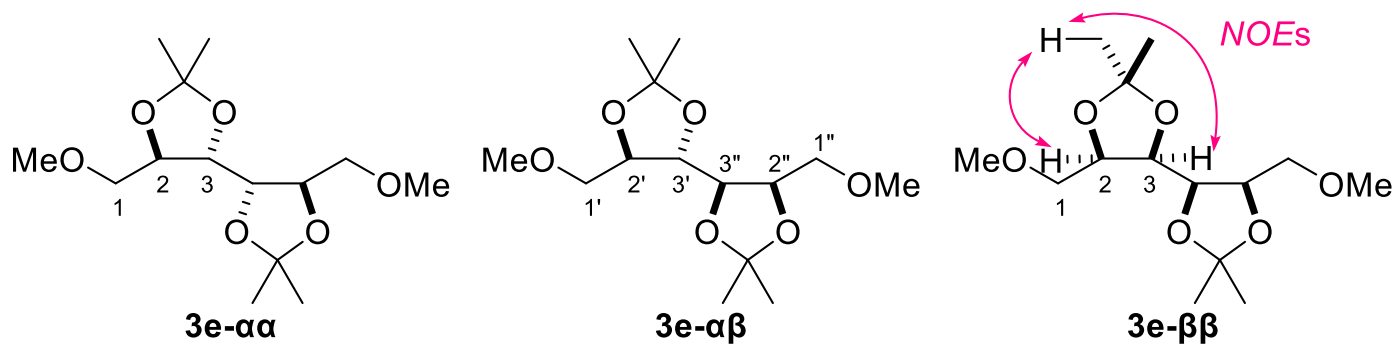

Dimer 3e. According to the general procedure A, a 1:1.5 diastereomeric mixture of $\mathbf{3 e - \alpha \alpha}$ and $\mathbf{3 e - \alpha} \boldsymbol{\beta}(14.9 \mathrm{mg}, 51.4 \mu \mathrm{mol})$, and $\mathbf{3 e - \beta} \boldsymbol{\beta}(3.7 \mathrm{mg}, 13 \mu \mathrm{mol})$ were synthesized from carboxylic acid 1e (44.1 mg, $232 \mu \mathrm{mol})$ by using MPT-623 $(92.7 \mathrm{mg}, 1.16 \mathrm{mmol})$ in EtOAc $(2.3 \mathrm{~mL})$. After being irradiated with stirring at $35{ }^{\circ} \mathrm{C}$ for $16 \mathrm{~h}$, the resultant mixture was filtered and concentrated. The residue was purified by automated flash column chromatography using the prepacked silica gel ( $7 \mathrm{~g}$, hexane/EtOAc $=70 / 30$ to $49 / 51)$. The combined yield of three diastereomers $3 \mathbf{e}$ was $55 \%$, and the $\alpha \alpha / \alpha \beta / \beta \beta$ ratio was 33:50:17. The C3-stereochemistry of $\mathbf{3 e - \beta} \boldsymbol{\beta}$ was determined by the NOESY experiment (Page S38).

3e- $\boldsymbol{\alpha} \boldsymbol{\alpha}$ and 3e- $\boldsymbol{\alpha} \boldsymbol{\beta}$ : colorless oil. ${ }^{1} \mathrm{H}$ NMR (400 MHz, $\left.\mathrm{CDCl}_{3}\right): \delta 4.42(1 \mathrm{H} \mathrm{x15/25}$, ddd, $J=8.7$, 6.0, $2.3 \mathrm{~Hz}), 4.26$ (2H x10/25, m, H2), 4.11 (1H x15/25, ddd, $J=8.7,6.4,2.3 \mathrm{~Hz}), 4.04(1 \mathrm{H}$ 
x15/25, dd, $J=9.6,6.0 \mathrm{~Hz}), 3.82(2 \mathrm{H} \mathrm{x10/25,} \mathrm{m,} \mathrm{H3),} 3.74(2 \mathrm{H} \mathrm{x15/25,} \mathrm{m),} 3.69$ (1H x15/25, $\mathrm{dd}, J=10.6,2.3 \mathrm{~Hz}), 3.55$ (1H x15/25, m), 3.53 (4H x10/25, m, H1), 3.47 (1H x15/25, dd, $J=$ 10.1, $6.4 \mathrm{~Hz}), 3.43(3 \mathrm{H} \mathrm{x} 15 / 25, \mathrm{~s}), 3.41(3 \mathrm{H} \mathrm{x} 15 / 25, \mathrm{~s}), 3.41\left(6 \mathrm{H} \times 10 / 25, \mathrm{~s}, \mathrm{C} 1-\mathrm{OCH}_{3}\right), 1.45$

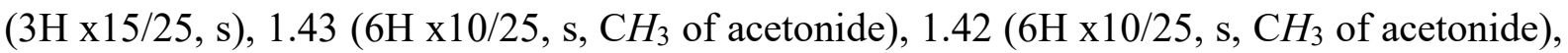
$1.41(3 \mathrm{H} \times 15 / 25, \mathrm{~s}), 1.37(3 \mathrm{H} \times 15 / 25, \mathrm{~s}), 1.36(3 \mathrm{H} \times 15 / 25, \mathrm{~s})$. Detectable signals of ${ }^{13} \mathrm{C}\left\{{ }^{1} \mathrm{H}\right\}$ NMR (100 MHz, $\left.\mathrm{CDCl}_{3}\right): \delta 109.75,109.69,109.2,80.3,78.3,77.0,77.0,76.1,73.7,73.2,73.0$, 71.0, 59.5, 59.4, 59.3, 27.7, 27.2, 27.1, 27.0, 26.6, 25.3. HRMS (ESI-TOF) m/z: $[\mathrm{M}+\mathrm{Na}]^{+} \mathrm{Calcd}$ for $\mathrm{C}_{14} \mathrm{H}_{26} \mathrm{O}_{6} \mathrm{Na} 313.1622$; Found 313.1633.

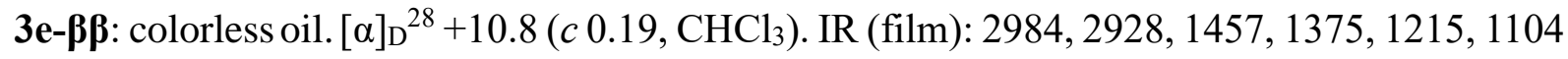
$\mathrm{cm}^{-1} .{ }^{1} \mathrm{H}$ NMR (500 MHz, C $\left.6 \mathrm{D}_{6}\right): \delta 4.41(2 \mathrm{H}, \mathrm{m}, \mathrm{H} 2), 4.33(2 \mathrm{H}, \mathrm{d}, J=5.2 \mathrm{~Hz}, \mathrm{H} 3), 3.65(2 \mathrm{H}$, dd, $J=9.2,5.0 \mathrm{~Hz}, \mathrm{H} 1), 3.49(2 \mathrm{H}, \mathrm{dd}, J=9.2,4.6 \mathrm{~Hz}, \mathrm{H} 1), 3.07\left(6 \mathrm{H}, \mathrm{s}, \mathrm{OCH}_{3}\right), 1.52(6 \mathrm{H}, \mathrm{s}$, $\mathrm{CH}_{3}$ of acetonide), $1.31\left(6 \mathrm{H}, \mathrm{s}, \mathrm{CH}_{3}\right.$ of acetonide). ${ }^{13} \mathrm{C}\left\{{ }^{1} \mathrm{H}\right\} \mathrm{NMR}\left(125 \mathrm{MHz}, \mathrm{C}_{6} \mathrm{D}_{6}\right): \delta 108.5$ (2C), 76.2 (2C), 75.4 (2C), 72.3 (2C), 58.7 (2C), 27.8 (2C), 25.6 (2C). HRMS (ESI-TOF) m/z: $[\mathrm{M}+\mathrm{Na}]^{+}$Calcd for $\mathrm{C}_{14} \mathrm{H}_{26} \mathrm{O}_{6} \mathrm{Na} 313.1622$; Found 313.1616.
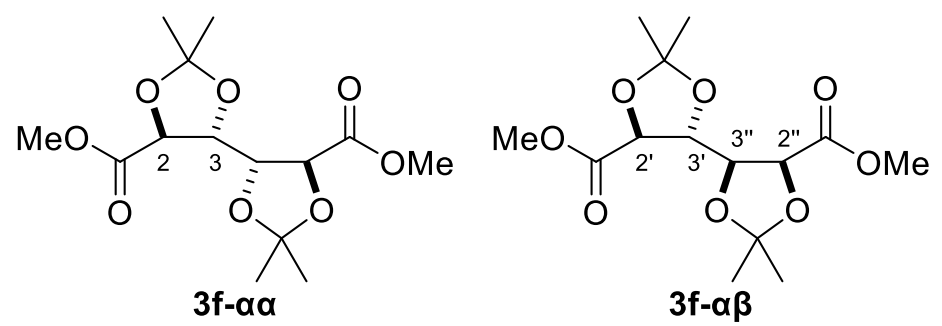

Dimer 3f. According to the general procedure A, a 1:1.2 diastereomeric mixture of $\mathbf{3 f}-\boldsymbol{\alpha \alpha}$ [CAS: 2377410-69-8] and 3f- $\boldsymbol{\beta}$ [CAS: 2377410-68-7] (9.00 mg, $28.3 \mu \mathrm{mol})$ was synthesized from carboxylic acid 1f [CAS: 67844-12-6] ${ }^{\mathrm{S} 6}$ (21.0 mg, $\left.103 \mu \mathrm{mol}\right)$ by using MPT-623 (82.4 $\mathrm{mg}, 1.03 \mathrm{mmol})$ in EtOAc $(1.0 \mathrm{~mL})$. After being irradiated with stirring at $35^{\circ} \mathrm{C}$ for $16 \mathrm{~h}$, the resultant mixture was filtered and concentrated. The residue was purified by automated column chromatography using the prepacked silica gel (16 g, hexane/EtOAc $=81 / 19$ to 60/40). The combined yield of two diastereomers $3 f$ was $56 \%$, and the $\alpha \alpha / \alpha \beta$ ratio was 45:55. The analytical data of $\mathbf{3 f}$ was identical to those reported previously. ${ }^{\mathrm{S} 7}$

${ }^{1} \mathrm{H}$ NMR (400 MHz, $\mathrm{CDCl}_{3}$ ): $\delta 4.76$ (1H x11/20, d, $J=6.4 \mathrm{~Hz}, \mathrm{H} 2$ '), 4.67 (2H x9/20, m, H2), 4.50 (1H x11/20, d, $J=5.5 \mathrm{~Hz}, \mathrm{H} 2$ "), 4.39 (1H x11/20, dd, $J=8.9,5.5 \mathrm{~Hz}, \mathrm{H} 3$ ”), 4.35 (2H x9/20, m, H3), 4.31 (2H x11/20, dd, $J=8.9,6.4$ Hz, H3'), 3.81 (6H x9/20, s, OCH3), 3.77 (3H

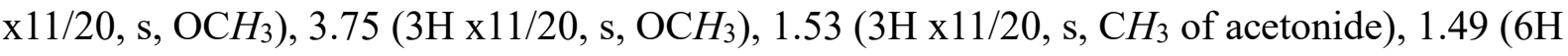
x9/20, s, $\mathrm{CH}_{3}$ of acetonide), $1.46\left(6 \mathrm{H} \mathrm{x9/20,} \mathrm{s,} \mathrm{CH}_{3}\right.$ of acetonide), $1.42\left(3 \mathrm{H} \mathrm{x11/20,} \mathrm{s,} \mathrm{CH}_{3}\right.$ of

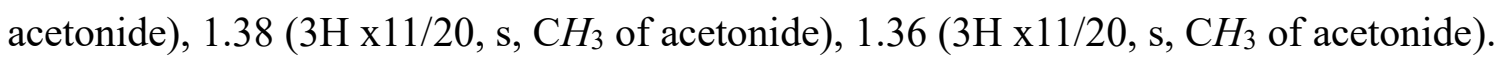




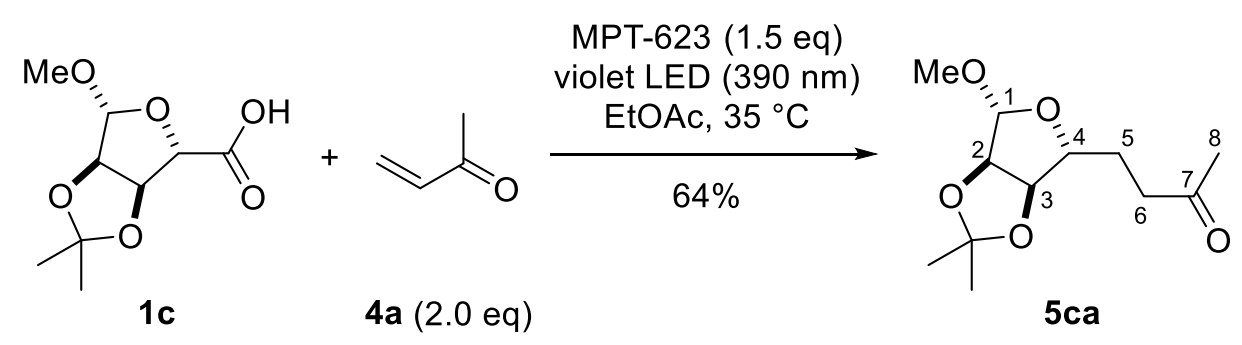

General Procedure B: Compound 5ca [CAS: 81691-16-9]. Argon was bubbled through a suspension of 1c $(21.8 \mathrm{mg}, 100 \mu \mathrm{mol})$ and MPT-623 $(12.0 \mathrm{mg}, 150 \mu \mathrm{mol})$ in EtOAc $(3.0 \mathrm{~mL})$ at $24{ }^{\circ} \mathrm{C}$ for $20 \mathrm{~min}$. Then, methyl vinyl ketone $4 \mathbf{a}(16.7 \mu \mathrm{L}, 200 \mu \mathrm{mol})$ was added to the suspension at $24^{\circ} \mathrm{C}$. The resultant mixture was sonicated for $3 \mathrm{~min}$, stirred, and then irradiated with a violet LED. After being irradiated with stirring at $35^{\circ} \mathrm{C}$ for $4 \mathrm{~h}$, the resultant mixture was filtered and concentrated. The residue was purified by automated column chromatography using the prepacked silica gel (16 g, hexane/EtOAc $=80 / 20$ to 59/41) to afford 5ca $(15.7 \mathrm{mg}, 64.3 \mu \mathrm{mol})$ in $64 \%$ yield. The analytical data of $\mathbf{5 c a}$ was identical to those reported previously. ${ }^{\mathrm{S} 3}$

${ }^{1} \mathrm{H}$ NMR (400 MHz, $\left.\mathrm{CDCl}_{3}\right): \delta 4.93(1 \mathrm{H}, \mathrm{s}, \mathrm{H} 1), 4.59(1 \mathrm{H}, \mathrm{d}, J=6.0 \mathrm{~Hz}, \mathrm{H} 2), 4.52(1 \mathrm{H}, \mathrm{d}, J=$ $6.0 \mathrm{~Hz}, \mathrm{H} 3), 4.12(1 \mathrm{H}, \mathrm{dd}, J=8.7,7.0 \mathrm{~Hz}, \mathrm{H} 4), 3.34\left(3 \mathrm{H}, \mathrm{s}, \mathrm{OCH}_{3}\right), 2.65-2.51(2 \mathrm{H}, \mathrm{m}, \mathrm{H} 6 \mathrm{a}$, 6b), 2.16 (3H, s, H8), 1.85-1.77 (2H, m, H5a, 5b), 1.47 (3H, s, $\mathrm{CH}_{3}$ of acetonide), 1.31 (3H, s, $\mathrm{CH}_{3}$ of acetonide).

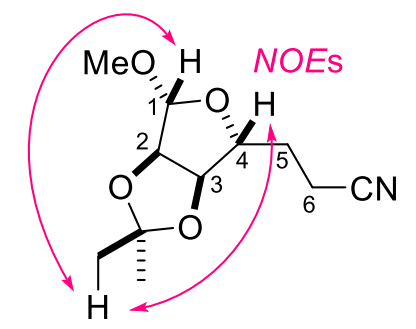

$5 \mathbf{c b}$

Compound 5cb. According to the general procedure B, 5cb $(7.5 \mathrm{mg}$, $33 \mu \mathrm{mol})$ was synthesized in $33 \%$ yield from $1 \mathrm{c}(21.8 \mathrm{mg}, 100 \mu \mathrm{mol})$ and acrylonitrile $\mathbf{4 b}(13.1 \mu \mathrm{L}, 200 \mu \mathrm{mol})$ by using MPT-623 (12.0 mg, $150 \mu \mathrm{mol})$ in EtOAc $(3.0 \mathrm{~mL})$. After being irradiated with stirring at $35{ }^{\circ} \mathrm{C}$ for $10 \mathrm{~h}$, the resultant mixture was filtered and concentrated. The residue was purified by flash column chromatography on silica gel $(5 \mathrm{~g}$, hexane/EtOAc $=5 / 1)$. The C4-stereochemistry of $\mathbf{5} \mathbf{c b}$ was determined by the NOESY experiment (Page S42): colorless oil. $[\alpha]_{\mathrm{D}}{ }^{25}-26.0$ (c 0.50, $\mathrm{CHCl}_{3}$ ). IR (film): 2938, 2248, 1449, 1378, 1210, 1100, 964, $866 \mathrm{~cm}^{-1} .{ }^{1} \mathrm{H}$ NMR (500 MHz, $\left.\mathrm{CDCl}_{3}\right): \delta 4.96(1 \mathrm{H}, \mathrm{s}, \mathrm{H1})$, $4.61(1 \mathrm{H}, \mathrm{d}, J=4.6 \mathrm{~Hz}, \mathrm{H} 2), 4.55(1 \mathrm{H}, \mathrm{d}, J=4.6 \mathrm{~Hz}, \mathrm{H} 3), 4.22(1 \mathrm{H}, \mathrm{t}, J=6.0 \mathrm{~Hz}, \mathrm{H} 4), 3.36$ $\left(3 \mathrm{H}, \mathrm{s}, \mathrm{OCH}_{3}\right), 2.56-2.42(2 \mathrm{H}, \mathrm{m}, \mathrm{H} 6), 1.91(2 \mathrm{H}, \mathrm{ddd}, J=6.0,6.0,6.0 \mathrm{~Hz}, \mathrm{H} 5), 1.48(3 \mathrm{H}, \mathrm{s}$, $\mathrm{CH}_{3}$ of acetonide), 1.32 (3H, s, $\mathrm{CH}_{3}$ of acetonide). ${ }^{13} \mathrm{C}\left\{{ }^{1} \mathrm{H}\right\} \mathrm{NMR}\left(125 \mathrm{MHz}, \mathrm{CDCl}_{3}\right): \delta 119.1$, 112.7, 110.0, 85.25, 85.23, 83.7, 55.5, 30.9, 26.4, 25.0, 14.3. HRMS (ESI-TOF) m/z: $[\mathrm{M}+\mathrm{Na}]^{+}$ Calcd for $\mathrm{C}_{11} \mathrm{H}_{17} \mathrm{NO}_{4} \mathrm{Na} 250.1050$; Found 250.1054. 


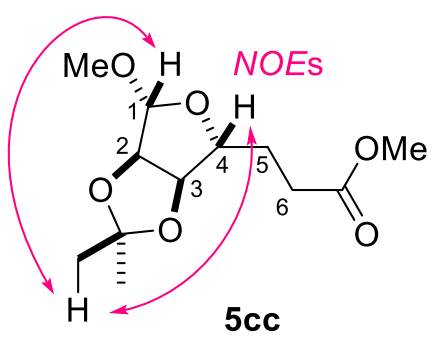

column chromatography on silica gel $(3 \mathrm{~g}$, hexane/EtOAc $=8 / 1)$ to afford 5cc. The C4stereochemistry of 5cc was determined by the NOESY experiment (Page S45): colorless oil. $[\alpha]_{\mathrm{D}}{ }^{21}-32.4$ (c 0.79, $\mathrm{CHCl}_{3}$ ). IR (film): 2943, 1734, 1443, 1376, 1247, 1203, 1167, 1099, 969 $\mathrm{cm}^{-1} .{ }^{1} \mathrm{H}$ NMR $\left(400 \mathrm{MHz}, \mathrm{CDCl}_{3}\right): \delta 4.94(1 \mathrm{H}, \mathrm{s}, \mathrm{H} 1), 4.60(1 \mathrm{H}, \mathrm{d}, J=5.9 \mathrm{~Hz}, \mathrm{H} 2), 4.53(1 \mathrm{H}$, d, $J=5.9 \mathrm{~Hz}, \mathrm{H} 3), 4.16(1 \mathrm{H}, \mathrm{t}, J=7.8 \mathrm{~Hz}, \mathrm{H} 4), 3.68\left(3 \mathrm{H}, \mathrm{s}, \mathrm{CO}_{2} \mathrm{CH}_{3}\right), 3.34\left(3 \mathrm{H}, \mathrm{s}, \mathrm{OCH}_{3}\right)$, 2.54-2.37 (2H, m, H6), 1.87 (2H, ddd, $J=7.8,7.8,7.8 \mathrm{~Hz}, \mathrm{H} 5), 1.47$ (3H, s, $\mathrm{CH}_{3}$ of acetonide), 1.31 (3H, s, $\mathrm{CH}_{3}$ of acetonide). ${ }^{13} \mathrm{C}\left\{{ }^{1} \mathrm{H}\right\} \mathrm{NMR}\left(100 \mathrm{MHz}, \mathrm{CDCl}_{3}\right): \delta 173.4,112.3,109.7,86.1$, 85.4, 84.0, 55.1, 51.7, 30.8, 30.2, 26.4, 24.9. HRMS (ESI-TOF) m/z: $[\mathrm{M}+\mathrm{Na}]^{+}$Calcd for $\mathrm{C}_{12} \mathrm{H}_{20} \mathrm{O}_{6} \mathrm{Na} 283.1152$; Found 283.1157.
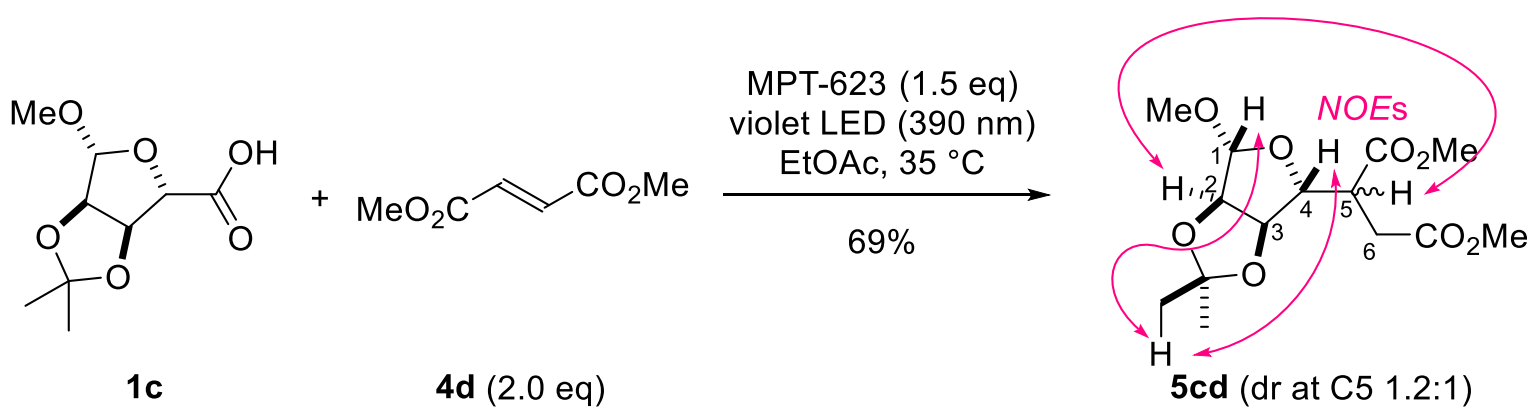

General Procedure C: Compound 5cd. Argon was bubbled through a suspension of 1c (21.8 mg, $100 \mu \mathrm{mol})$, dimethyl fumarate $4 \mathbf{d}(28.8 \mathrm{mg}, 200 \mu \mathrm{mol})$, and MPT-623 (12.0 mg, 150 $\mu \mathrm{mol})$ in EtOAc $(3.0 \mathrm{~mL})$ at $24{ }^{\circ} \mathrm{C}$ for $20 \mathrm{~min}$. The resultant mixture was sonicated for $3 \mathrm{~min}$, stirred, and then irradiated with a violet LED. After being irradiated with stirring at $35^{\circ} \mathrm{C}$ for $16 \mathrm{~h}$, the resultant mixture was filtered and concentrated. The residue was purified by flash column chromatography on silica gel $(5 \mathrm{~g}$, hexane/EtOAc $=4 / 1)$ to afford a 1.2:1 diastereomeric mixture of 5cd $(22.0 \mathrm{mg}, 69.1 \mu \mathrm{mol})$ in $69 \%$ yield. The C4-stereochemistry of 5cd was determined by the NOESY experiment (Page S48). The analytical data of 5cd were collected by using 1.2:1 diastereomeric mixture: colorless oil. ${ }^{1} \mathrm{H} \mathrm{NMR}\left(500 \mathrm{MHz}, \mathrm{CDCl}_{3}\right): \delta 4.95(1 \mathrm{H}$ x10/22, s, H1), $4.93(1 \mathrm{H} \mathrm{x12/22,} \mathrm{s,} \mathrm{H1),} 4.78$ (1H x12/22 and $1 \mathrm{H} \mathrm{x10/22,} \mathrm{br} \mathrm{d,} J=5.7 \mathrm{~Hz}, \mathrm{H} 2)$, $4.59(1 \mathrm{H} \mathrm{x} 10 / 22, \mathrm{~d}, J=5.7 \mathrm{~Hz}, \mathrm{H} 3), 4.55(1 \mathrm{H} \mathrm{x} 12 / 22, \mathrm{~d}, J=6.3 \mathrm{~Hz}, \mathrm{H} 3), 4.27(1 \mathrm{H} \mathrm{x} 12 / 22, \mathrm{~d}$, $J=8.6 \mathrm{~Hz}, \mathrm{H} 4), 4.25(1 \mathrm{H} \times 10 / 22, \mathrm{~d}, J=10.9 \mathrm{~Hz}, \mathrm{H} 4), 3.74\left(3 \mathrm{H} \mathrm{x} 10 / 22, \mathrm{~s}, \mathrm{CO}_{2} \mathrm{CH}_{3}\right), 3.72(3 \mathrm{H}$ x12/22, s, $\left.\mathrm{CO}_{2} \mathrm{CH}_{3}\right), 3.68\left(3 \mathrm{H} \mathrm{x} 12 / 22, \mathrm{~s}, \mathrm{CO}_{2} \mathrm{CH}_{3}\right), 3.67\left(3 \mathrm{H} \mathrm{x} 10 / 22, \mathrm{~s}, \mathrm{CO}_{2} \mathrm{CH}_{3}\right), 3.34(3 \mathrm{H}$ x10/22, s, $\left.\mathrm{OCH}_{3}\right), 3.32$ ( $\left.3 \mathrm{H} \times 12 / 22, \mathrm{~s}, \mathrm{OCH}_{3}\right), 3.07$ (1H x12/22, ddd, $\left.J=12.6,8.1,4.1 \mathrm{~Hz}, \mathrm{H} 5\right)$, 
$2.99(1 \mathrm{H} \times 10 / 22$, ddd, $J=10.9,10.9,4.0 \mathrm{~Hz}, \mathrm{H} 5), 2.80(2 \mathrm{H} \times 10 / 22$ and $1 \mathrm{H} \times 12 / 22, \mathrm{~m}, \mathrm{H} 6 \mathrm{a}$, H6b, H6b), 2.53 (1H x12/22, dd, $J=13.3,3.2 \mathrm{~Hz}, \mathrm{H} 6 \mathrm{a}), 1.46(3 \mathrm{H} \mathrm{x} 12 / 22$ and $3 \mathrm{H} \mathrm{x} 10 / 22$, s,

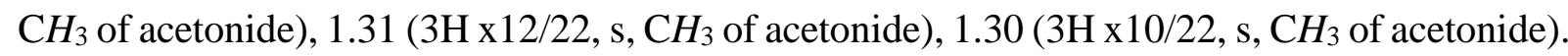
${ }^{13} \mathrm{C}\left\{{ }^{1} \mathrm{H}\right\} \mathrm{NMR}\left(125 \mathrm{MHz}, \mathrm{CDCl}_{3}\right): \delta 172.5,172.1,171.7$ (2C), 112.7, 112.6, 110.1, 109.8, 88.0, 86.3, 85.4, 85.0, 82.6, 82.3, 55.8, 55.7, 52.3, 52.1, 52.0, 51.9, 46.12, 46.07, 33.8, 33.7, 26.6, 26.5, 25.1, 25.0. HRMS (ESI-TOF) $\mathrm{m} / \mathrm{z}$ : $[\mathrm{M}+\mathrm{Na}]^{+}$Calcd for $\mathrm{C}_{14} \mathrm{H}_{22} \mathrm{O}_{8} \mathrm{Na}$ 341.1207; Found 341.1215 .

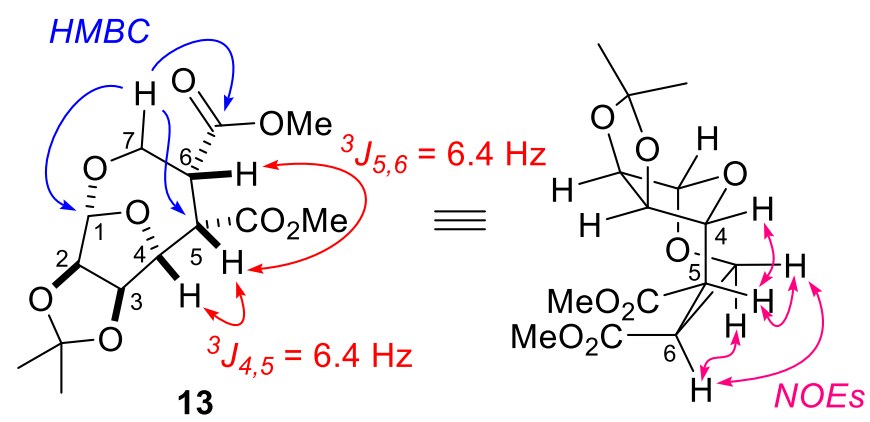

Compound 13. According to the general procedure C, $13(10.5 \mathrm{mg}, 33.2 \mu \mathrm{mol})$ was synthesized in 33\% yield from 1c $(21.8 \mathrm{mg}, 100 \mu \mathrm{mol})$ and dimethyl acetylenedicarboxylate $4 \mathbf{e}$ $(24.5 \mu \mathrm{L}, 200 \mu \mathrm{mol})$ by using MPT-623 $(12.0 \mathrm{mg}, 150 \mu \mathrm{mol})$ in EtOAc $(3.0 \mathrm{~mL})$. After being irradiated with stirring at $35{ }^{\circ} \mathrm{C}$ for $5 \mathrm{~h}$, the resultant mixture was filtered and concentrated. The residue was purified by PTLC $(20 \mathrm{~cm} \times 10 \mathrm{~cm}, 2$ plates, hexane/EtOAc $=4 / 1)$. The C4, C5, C6-stereochemistries of $\mathbf{1 3}$ were determined by the NOESY experiment (Page S52): colorless oil. $[\alpha]_{\mathrm{D}}{ }^{23}+5.53\left(c 1.01, \mathrm{CHCl}_{3}\right)$. IR (film): 2952, 1739, 1442, 1379, 1207, 1128, 1088 , $1036 \mathrm{~cm}^{-1} .{ }^{1} \mathrm{H}$ NMR $\left(400 \mathrm{MHz}, \mathrm{CDCl}_{3}\right): \delta 5.30(1 \mathrm{H}, \mathrm{s}, \mathrm{H} 1), 5.29(1 \mathrm{H}, \mathrm{d}, J=6.0 \mathrm{~Hz}, \mathrm{H} 3), 4.96$ $(1 \mathrm{H}, \mathrm{d}, J=6.4 \mathrm{~Hz}, \mathrm{H} 4), 4.41(1 \mathrm{H}, \mathrm{d}, J=6.0 \mathrm{~Hz}, \mathrm{H} 2), 4.16(1 \mathrm{H}, \mathrm{dd}, J=12.4,1.4 \mathrm{~Hz}, \mathrm{H} 7 \mathrm{a}), 4.03$ $(1 \mathrm{H}, \mathrm{dd}, J=12.4,3.6 \mathrm{~Hz}, \mathrm{H} 7 \mathrm{~b}), 3.75\left(3 \mathrm{H}, \mathrm{s}, \mathrm{CO}_{2} \mathrm{CH}_{3}\right), 3.71\left(3 \mathrm{H}, \mathrm{s}, \mathrm{CO}_{2} \mathrm{CH}_{3}\right), 3.33(1 \mathrm{H}, \mathrm{m}$, H6), $3.14(1 \mathrm{H}, \mathrm{dd}, J=6.4,6.4 \mathrm{~Hz}, \mathrm{H} 5), 1.48\left(3 \mathrm{H}, \mathrm{s}, \mathrm{CH}_{3}\right.$ of acetonide), 1.32 (3H, s, $\mathrm{CH}_{3}$ of acetonide). ${ }^{13} \mathrm{C}\left\{{ }^{1} \mathrm{H}\right\}$ NMR $\left(100 \mathrm{MHz}, \mathrm{CDCl}_{3}\right): \delta 172.2,171.0,111.9,105.1,88.0,86.2,81.5$, 61.7, 52.2 (2C), 48.0, 46.0, 26.4, 24.9. HRMS (ESI-TOF) m/z: [M+Na] $]^{+}$Calcd for $\mathrm{C}_{14} \mathrm{H}_{20} \mathrm{O}_{8} \mathrm{Na}$ 339.1050; Found 339.1043.

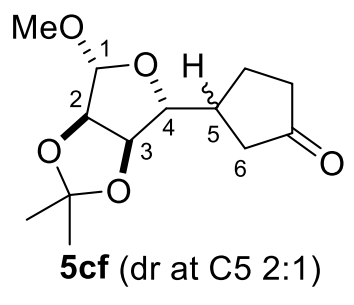

Compound 5cf [CAS: 1808118-86-6, 1808118-87-7]. According to the general procedure B, a 2:1 diastereomeric mixture of $\mathbf{5 c f}(14.3 \mathrm{mg}$, $55.8 \mu \mathrm{mol})$ was synthesized in $56 \%$ yield from $1 \mathrm{c}(21.8 \mathrm{mg}, 100 \mu \mathrm{mol})$ and cyclopentenone $\mathbf{4 f}(16.7 \mu \mathrm{L}, 199 \mu \mathrm{mol})$ by using MPT-623 $(12.0 \mathrm{mg}$, $150 \mu \mathrm{mol})$ in EtOAc $(3.0 \mathrm{~mL})$. After being irradiated with stirring at $35{ }^{\circ} \mathrm{C}$ for $16 \mathrm{~h}$, the resultant mixture was filtered and concentrated. The residue was purified 
by automated column chromatography using the prepacked silica gel $(7 \mathrm{~g}$, hexane/EtOAc $=$ $73 / 27$ to $52 / 48)$. The analytical data of $\mathbf{5} \mathbf{c f}$ was identical to those reported previously. ${ }^{\mathrm{S} 3}$

${ }^{1} \mathrm{H}$ NMR (400 MHz, $\left.\mathrm{CDCl}_{3}\right): \delta 4.98(1 \mathrm{H} \mathrm{x} 2 / 3, \mathrm{~s}, \mathrm{H} 1), 4.95(1 \mathrm{H} \mathrm{x1/3}, \mathrm{s}, \mathrm{H} 1), 4.64(1 \mathrm{H} \mathrm{x1/3}, \mathrm{d}$, $J=6.4, \mathrm{~Hz}, \mathrm{H} 3), 4.61(1 \mathrm{H} \mathrm{x1} / 3, \mathrm{~d}, J=2.3 \mathrm{~Hz}, \mathrm{H} 2), 4.60(1 \mathrm{H} \mathrm{x} 2 / 3, \mathrm{~d}, J=2.3 \mathrm{~Hz}, \mathrm{H} 2), 4.52(1 \mathrm{H}$ $\mathrm{x} 2 / 3, \mathrm{~d}, J=6.0 \mathrm{~Hz}, \mathrm{H} 3), 3.99(1 \mathrm{H} \mathrm{x} 3 / 3, \mathrm{dd}, J=10.3,4.3 \mathrm{~Hz}, \mathrm{H} 4), 3.40\left(3 \mathrm{H} \mathrm{x} 2 / 3, \mathrm{~s}, \mathrm{OCH}_{3}\right)$, $3.34\left(3 \mathrm{H} \mathrm{x} 1 / 3, \mathrm{~s}, \mathrm{OCH}_{3}\right), 2.43-2.15(6 \mathrm{H}, \mathrm{m}), 1.98-1.63(2 \mathrm{H}, \mathrm{m}), 1.49\left(3 \mathrm{H} \mathrm{x} 3 / 3, \mathrm{~s}, \mathrm{CH}_{3}\right.$ of

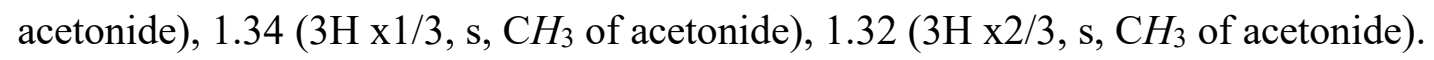
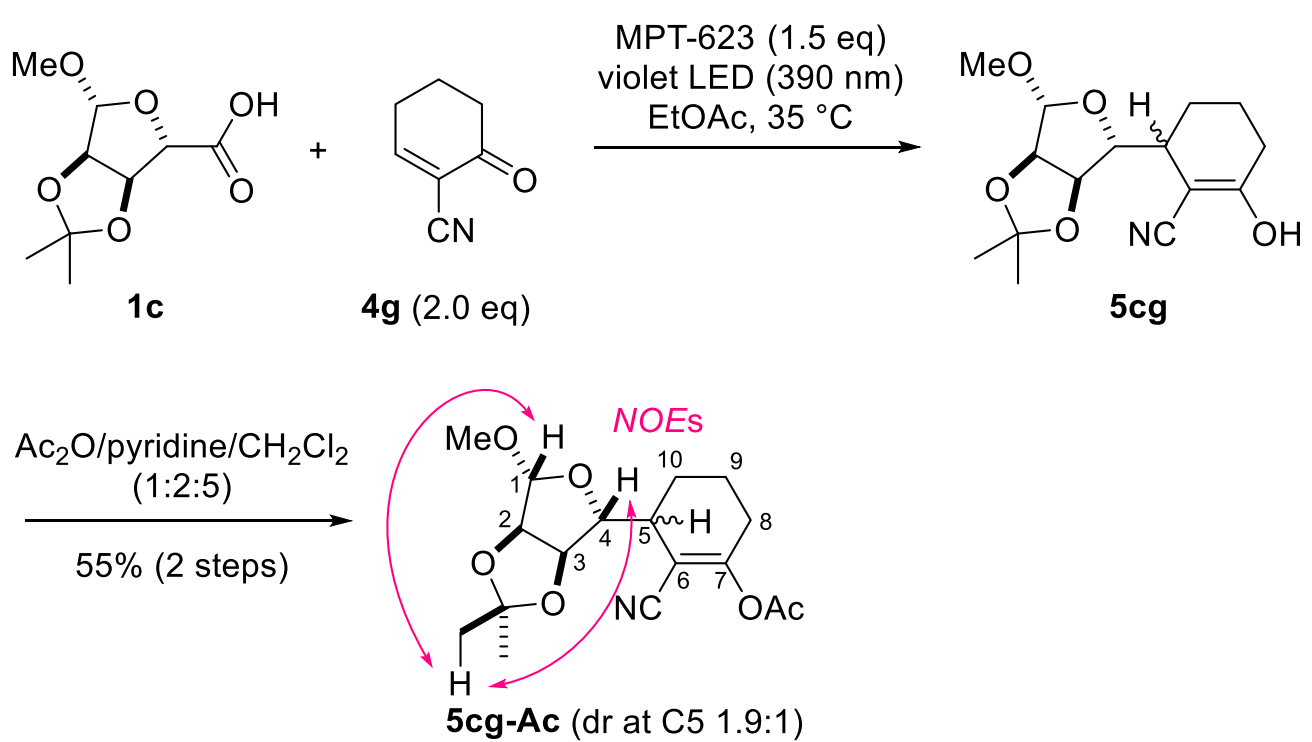

Acetate 5cg-Ac. According to the general procedure $\mathrm{C}$, a diastereomeric mixture of $\mathbf{5 c g}$ was synthesized from 1c $(21.8 \mathrm{mg}, 100 \mu \mathrm{mol})$ and cyclohexenone $4 \mathrm{~g}$ [CAS: 91624-93-0] ${ }^{\mathrm{S} 8}$ (24.2 $\mathrm{mg}, 200 \mu \mathrm{mol})$ by using MPT-623 $(12.0 \mathrm{mg}, 150 \mu \mathrm{mol})$ in EtOAc $(3.0 \mathrm{~mL})$. After being irradiated with stirring at $35{ }^{\circ} \mathrm{C}$ for $7 \mathrm{~h}$, the resultant mixture was filtered and concentrated. The residue was filtered through a pad of silica gel ( $3 \mathrm{~g}$, hexane/EtOAc $=5 / 1$ to $4 / 1$ to $3 / 1$ to $2 / 1)$ to afford the crude $\mathbf{5} \mathbf{c g}(31.5 \mathrm{mg})$, which was used in the next reaction without further purification. HRMS (ESI-TOF) $\mathrm{m} / \mathrm{z}$ : $[\mathrm{M}+\mathrm{Na}]^{+}$Calcd for $\mathrm{C}_{15} \mathrm{H}_{21} \mathrm{NO}_{5} \mathrm{Na}$ 318.1312; Found 318.1305 .

The above crude $5 \mathrm{cg}(31.5 \mathrm{mg})$ was dissolved in $\mathrm{CH}_{2} \mathrm{Cl}_{2}(1.5 \mathrm{~mL})$, pyridine $(0.60 \mathrm{~mL})$, and $\mathrm{Ac}_{2} \mathrm{O}(0.30 \mathrm{~mL})$ at $25^{\circ} \mathrm{C}$. After the reaction mixture was stirred at $25^{\circ} \mathrm{C}$ for $4 \mathrm{~h}, \mathrm{H}_{2} \mathrm{O}(1 \mathrm{~mL})$ was added. The resultant mixture was extracted with $\mathrm{CH}_{2} \mathrm{Cl}_{2}(1 \mathrm{~mL} \times 3)$. The combined organic layers were dried over $\mathrm{Na}_{2} \mathrm{SO}_{4}$, filtered, and concentrated. The residue was purified by flash column chromatography on silica gel ( $3 \mathrm{~g}$, hexane/EtOAc $=10 / 1$ to $8 / 1$ to $5 / 1$ to $3 / 1$ ) to afford a 1.9:1 diastereomeric mixture of 5cg-Ac $(18.6 \mathrm{mg}, 55.2 \mu \mathrm{mol})$ in $55 \%$ yield. To collect the analytical data, 5cg-Ac was partially purified by PTLC $(10 \mathrm{~cm} \times 10 \mathrm{~cm}$, hexane/EtOAc = 1/1). The C4-stereochemistries of the major isomer and the minor isomer of 5cg-Ac were determined by the NOESY experiments, respectively (Pages S56 and S59). 
Major isomer of 5cg-Ac: colorless oil. $[\alpha]_{\mathrm{D}}{ }^{26}-56.4$ (c 1.01, $\left.\mathrm{CHCl}_{3}\right)$. IR (film): 2985, 2217, $1772,1651,1452,1375,1185,1094 \mathrm{~cm}^{-1} .{ }^{1} \mathrm{H}$ NMR (400 MHz, $\left.\mathrm{CDCl}_{3}\right): \delta 5.08(1 \mathrm{H}, \mathrm{dd}, J=6.0$, $1.4 \mathrm{~Hz}, \mathrm{H} 3), 4.96(1 \mathrm{H}, \mathrm{s}, \mathrm{H} 1), 4.61(1 \mathrm{H}, \mathrm{d}, J=6.0 \mathrm{~Hz}, \mathrm{H} 2), 4.21$ (1H, dd, $J=9.6,1.4 \mathrm{~Hz}, \mathrm{H} 4)$, $3.37\left(3 \mathrm{H}, \mathrm{s}, \mathrm{OCH}_{3}\right), 2.60(1 \mathrm{H}, \mathrm{m}, \mathrm{H} 5), 2.44-2.28(2 \mathrm{H}, \mathrm{m}, \mathrm{H} 8), 2.24\left(3 \mathrm{H}, \mathrm{s}, \mathrm{CH}_{3} \mathrm{CO}\right), 2.03(1 \mathrm{H}$, m, H10a), 1.88 (1H, m, H9a), $1.75(1 \mathrm{H}, \mathrm{m}, \mathrm{H} 9 \mathrm{~b}), 1.65(1 \mathrm{H}, \mathrm{m}, \mathrm{H} 10 \mathrm{~b}), 1.49\left(3 \mathrm{H}, \mathrm{s}, \mathrm{CH}_{3}\right.$ of acetonide), 1.33 (3H, s, $\mathrm{CH}_{3}$ of acetonide). ${ }^{13} \mathrm{C}\left\{{ }^{1} \mathrm{H}\right\} \mathrm{NMR}\left(100 \mathrm{MHz}, \mathrm{CDCl}_{3}\right): \delta 167.5,165.4$, $116.1,112.3$, 109.8, 101.6, 87.8, 85.4, 81.7, 55.7, 38.9, 28.1, 26.6, 25.0, 22.7, 20.8, 17.5. HRMS (ESI-TOF) m/z: [M+Na] ${ }^{+}$Calcd for $\mathrm{C}_{17} \mathrm{H}_{23} \mathrm{NO}_{6} \mathrm{Na} 360.1418$; Found 360.1415.

Minor isomer of 5cg-Ac: colorless oil. $[\alpha]_{\mathrm{D}}{ }^{24}+27.3$ (c 1.03, $\mathrm{CHCl}_{3}$ ). IR (film): 2939, 2221, 1770, 1650, 1452, 1374, 1192, $1096 \mathrm{~cm}^{-1} .{ }^{1} \mathrm{H}$ NMR (400 MHz, $\left.\mathrm{CDCl}_{3}\right): \delta 5.05(1 \mathrm{H}, \mathrm{s}, \mathrm{H1}), 4.69$ $(1 \mathrm{H}, \mathrm{dd}, J=6.0,2.7 \mathrm{~Hz}, \mathrm{H} 3), 4.56(1 \mathrm{H}, \mathrm{d}, J=6.0 \mathrm{~Hz}, \mathrm{H} 2), 4.14(1 \mathrm{H}, \mathrm{dd}, J=9.6,2.8 \mathrm{~Hz}, \mathrm{H} 4)$, $3.50\left(3 \mathrm{H}, \mathrm{s}, \mathrm{OCH}_{3}\right), 2.60(1 \mathrm{H}, \mathrm{m}, \mathrm{H} 5), 2.41-2.29(2 \mathrm{H}, \mathrm{m}, \mathrm{H} 8), 2.25\left(3 \mathrm{H}, \mathrm{s}, \mathrm{CH}_{3} \mathrm{CO}\right), 1.93-1.82$ (2H, m, H9a, H10a), $1.74(1 \mathrm{H}, \mathrm{m}, \mathrm{H} 9 \mathrm{~b}), 1.60(1 \mathrm{H}, \mathrm{m}, \mathrm{H} 10 \mathrm{~b}), 1.50$ (3H, s, $\mathrm{CH}_{3}$ of acetonide), $1.33\left(3 \mathrm{H}, \mathrm{s}, \mathrm{CH}_{3}\right.$ of acetonide). ${ }^{13} \mathrm{C}\left\{{ }^{1} \mathrm{H}\right\} \mathrm{NMR}\left(100 \mathrm{MHz}, \mathrm{CDCl}_{3}\right): \delta 167.8,164.3,116.0,113.1$, 109.3, 103.2, 90.2, 85.1, 82.2, 56.5, 39.8, 28.3, 26.8, 25.3, 24.0, 20.8, 18.8. HRMS (ESI-TOF) $\mathrm{m} / \mathrm{z}:[\mathrm{M}+\mathrm{Na}]^{+}$Calcd for $\mathrm{C}_{17} \mathrm{H}_{23} \mathrm{NO}_{6} \mathrm{Na} 360.1418$; Found 360.1403 .

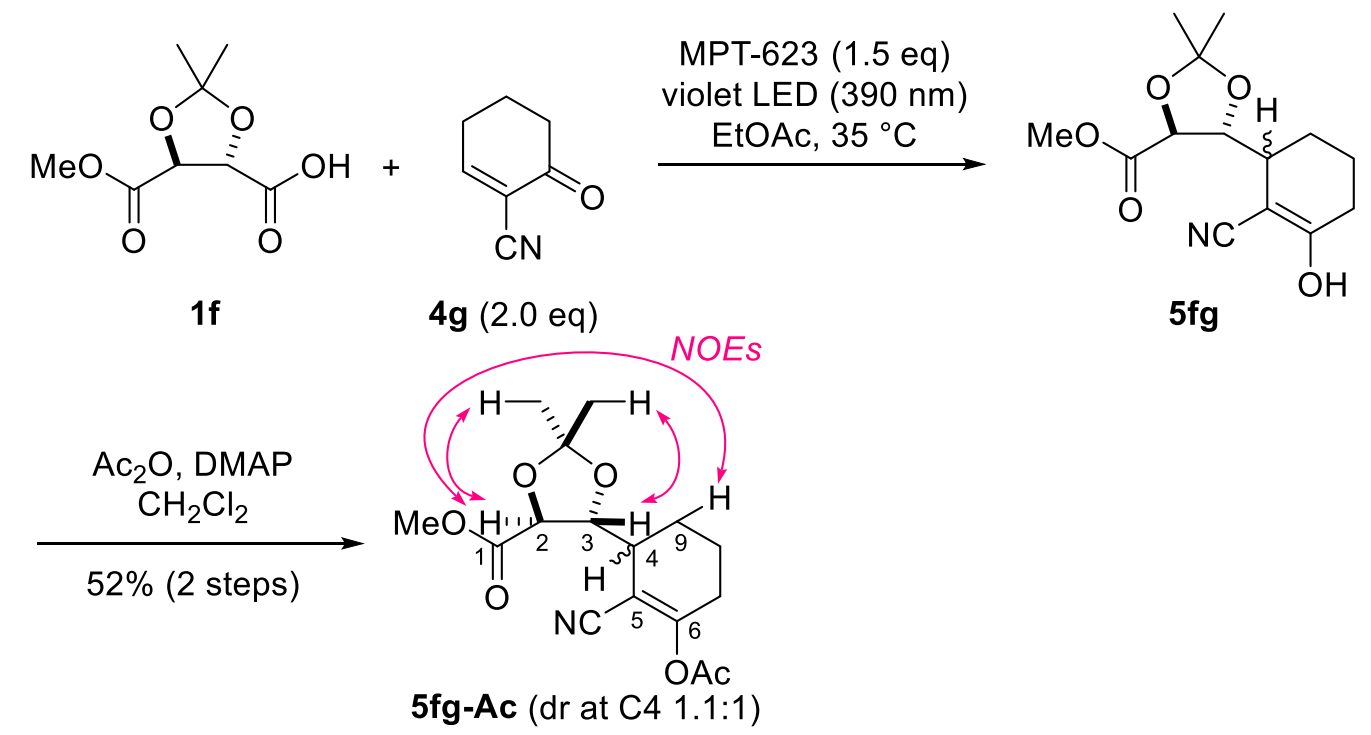

Acetate 5fg-Ac. According to the general procedure $\mathrm{C}$, a diastereomeric mixture of $\mathbf{5 f g}$ was synthesized from $\mathbf{1 f}(22.0 \mathrm{mg}, 108 \mu \mathrm{mol})$ and $\mathbf{4 g}(26.1 \mathrm{mg}, 215 \mu \mathrm{mol})$ by using MPT-623 (12.9 $\mathrm{mg}, 161 \mu \mathrm{mol})$ in EtOAc $(3.3 \mathrm{~mL})$. After being irradiated with stirring at $35^{\circ} \mathrm{C}$ for $14 \mathrm{~h}$, the resultant mixture was filtered and concentrated. The residue was filtered through a pad of silica gel ( $3 \mathrm{~g}$, hexane/EtOAc $=3 / 1$ to $2 / 1)$ to afford the crude $\mathbf{5 f g}(24.7 \mathrm{mg})$, which was used in the next reaction without further purification. HRMS (ESI-TOF) $\mathrm{m} / \mathrm{z}:[\mathrm{M}+\mathrm{Na}]^{+} \mathrm{Calcd}$ for $\mathrm{C}_{14} \mathrm{H}_{19} \mathrm{NO}_{5} \mathrm{Na}$ 304.1155; Found 304.1154

$\mathrm{Ac}_{2} \mathrm{O}(166 \mu \mathrm{L}, 1.76 \mathrm{mmol})$ and 4-dimethylaminopyridine (DMAP, $\left.10.7 \mathrm{mg}, 87.6 \mu \mathrm{mol}\right)$ were 
added to a solution of the above crude $\mathbf{5 f g}(24.7 \mathrm{mg})$ in $\mathrm{CH}_{2} \mathrm{Cl}_{2}(0.88 \mathrm{~mL})$ at $27{ }^{\circ} \mathrm{C}$. After the reaction mixture was stirred at $27^{\circ} \mathrm{C}$ for $3 \mathrm{~h}, \mathrm{H}_{2} \mathrm{O}(1 \mathrm{~mL})$ was added. The resultant mixture was extracted with $\mathrm{CH}_{2} \mathrm{Cl}_{2}(1 \mathrm{~mL} \times 3)$. The combined organic layers were dried over $\mathrm{Na}_{2} \mathrm{SO}_{4}$, filtered, and concentrated. The residue was purified by flash column chromatography on silica gel $(6 \mathrm{~g}$, hexane/EtOAc $=6 / 1$ to $5 / 1$ to $4 / 1$ to $1 / 1)$ to afford a $1.1: 1$ diastereomeric mixture of $\mathbf{5 f g}-\mathbf{A c}(18.0 \mathrm{mg}, 55.7 \mu \mathrm{mol})$ in $52 \%$ yield. To collect the analytical data, $\mathbf{5 f g}$-Ac was partially purified by PTLC $(20 \mathrm{~cm} \times 10 \mathrm{~cm}$, hexane/EtOAc $=1 / 1)$. The analytical data of $\mathbf{5 f g}$-Ac was collected by using a 1.3:1 diastereomeric mixture. The C3-stereochemistries of the major isomer and the minor isomer of $\mathbf{5 f g}$-Ac were determined by the NOESY experiment (Page S61). 5fg-Ac: colorless oil. ${ }^{1} \mathrm{H}$ NMR (400 MHz, $\left.\mathrm{CDCl}_{3}\right)$ : $\delta 4.50(1 \mathrm{H} \mathrm{x13} / 23$, dd, $J=7.8,3.2 \mathrm{~Hz}, \mathrm{H} 3)$, $4.46(1 \mathrm{H} \mathrm{x} 10 / 23, \mathrm{~d}, J=6.4 \mathrm{~Hz}, \mathrm{H} 2), 4.38(1 \mathrm{H}, \mathrm{m}), 3.80$ (3H x13/23, s), 3.79 (3H x10/23, s), $2.82(1 \mathrm{H}, \mathrm{m}), 2.40-2.32(2 \mathrm{H}, \mathrm{m}), 2.25(3 \mathrm{H}, \mathrm{s}), 2.01-1.85(1 \mathrm{H}, \mathrm{m}), 1.85-1.68(2 \mathrm{H}, \mathrm{m}), 1.62(1 \mathrm{H}$, $\mathrm{m}), 1.52(3 \mathrm{H} \times 10 / 23, \mathrm{~s}), 1.48(3 \mathrm{H} \mathrm{x} 13 / 23, \mathrm{~s}), 1.43(3 \mathrm{H} \times 10 / 23, \mathrm{~s}), 1.42(3 \mathrm{H} \times 13 / 23, \mathrm{~s}) .{ }^{13} \mathrm{C}\left\{{ }^{1} \mathrm{H}\right\}$ NMR (100 MHz, $\left.\mathrm{CDCl}_{3}\right): \delta 171.2,171.0,167.5$ (2C), 164.6 (2C), 115.5, 114.9, 112.0, 111.4, 102.2, 101.5, 80.9, 78.9, 77.0, 75.7, 52.62, 52.57, 38.5, 37.0, 28.10, 28.08, 26.8, 26.4, 25.7, 25.2, 23.9, 21.0, 20.8 (2C), 19.6, 18.9. HRMS (ESI-TOF) $\mathrm{m} / \mathrm{z}:[\mathrm{M}+\mathrm{Na}]^{+}$Calcd for $\mathrm{C}_{16} \mathrm{H}_{21} \mathrm{NO}_{6} \mathrm{Na} 346.1261$; Found 346.1252.

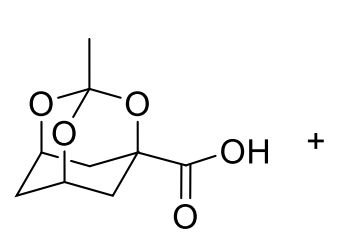

$1 \mathrm{~g}$<smiles>N#CC1=CCCCC1=O</smiles>

$\mathbf{4 g}(2.0 \mathrm{eq})$
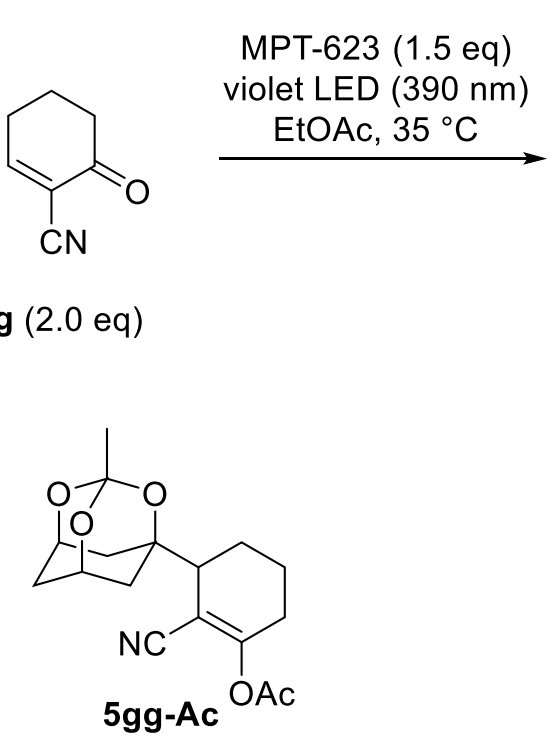

Acetate 5gg-Ac. According to the general procedure C, $\mathbf{5 g g}$ was synthesized from 1g [CAS: 1326319-29-2] ${ }^{\mathrm{S} 9}$ (20.0 mg, $\left.99.9 \mu \mathrm{mol}\right)$ and $\mathbf{4 g}(24.2 \mathrm{mg}, 200 \mu \mathrm{mol})$ by using MPT-623 (12.0 $\mathrm{mg}, 150 \mu \mathrm{mol})$ in EtOAc $(3.0 \mathrm{~mL})$. After being irradiated with stirring at $35^{\circ} \mathrm{C}$ for $14 \mathrm{~h}$, the resultant mixture was filtered and concentrated. The residue was filtered through a pad of silica gel $(5 \mathrm{~g}$, hexane/EtOAc $=1 / 1)$ to afford the crude $\mathbf{5 g g}(22.0 \mathrm{mg})$, which was used in the next reaction without further purification. HRMS (ESI-TOF) $\mathrm{m} / \mathrm{z}:[\mathrm{M}+\mathrm{Na}]^{+} \mathrm{Calcd}$ for $\mathrm{C}_{15} \mathrm{H}_{19} \mathrm{NO}_{4} \mathrm{Na} 300.1206$; Found 300.1206. 
$\mathrm{Ac}_{2} \mathrm{O}(150 \mu \mathrm{L}, 1.59 \mathrm{mmol})$ and DMAP $(9.7 \mathrm{mg}, 79 \mu \mathrm{mol})$ were added to a solution of the above crude $5 \mathrm{gg}(22.0 \mathrm{mg})$ in $\mathrm{CH}_{2} \mathrm{Cl}_{2}(0.80 \mathrm{~mL})$ at $25^{\circ} \mathrm{C}$. After the reaction mixture was stirred at $25^{\circ} \mathrm{C}$ for $4 \mathrm{~h}, \mathrm{H}_{2} \mathrm{O}(1 \mathrm{~mL})$ was added. The resultant mixture was extracted with $\mathrm{CH}_{2} \mathrm{Cl}_{2}(1$ $\mathrm{mL} \times 3$ ). The combined organic layers were dried over $\mathrm{Na}_{2} \mathrm{SO}_{4}$, filtered, and concentrated. The residue was purified by flash column chromatography on silica gel ( $3 \mathrm{~g}$, hexane/EtOAc $=$ $2 / 1$ to $1 / 1)$ to afford $\mathbf{5 g g}$-Ac $(18.9 \mathrm{mg}, 59.2 \mu \mathrm{mol})$ in $59 \%$ yield over 2 steps: yellow solid. m.p. 142-143 ${ }^{\circ}$ C. IR (film): 2952, 2369, 2220, 1767, 1646, 1390, 1365, 1324, 1133, $1009 \mathrm{~cm}^{-1} .{ }^{1} \mathrm{H}$ NMR (400 MHz, $\left.\mathrm{CDCl}_{3}\right): \delta 4.40(2 \mathrm{H}, \mathrm{m}), 2.58-2.47(2 \mathrm{H}, \mathrm{m}), 2.40-2.24(4 \mathrm{H}, \mathrm{m}), 2.25(3 \mathrm{H}, \mathrm{s})$, $1.92(1 \mathrm{H}, \mathrm{m}), 1.86-1.76(2 \mathrm{H}, \mathrm{m}), 1.71(1 \mathrm{H}, \mathrm{d}, J=11.4 \mathrm{~Hz}), 1.65(1 \mathrm{H}, \mathrm{m}), 1.57(1 \mathrm{H}, \mathrm{d}, J=11.5$ $\mathrm{Hz}), 1.48(1 \mathrm{H}, \mathrm{m}), 1.46(3 \mathrm{H}, \mathrm{s}) .{ }^{13} \mathrm{C}\left\{{ }^{1} \mathrm{H}\right\} \mathrm{NMR}\left(100 \mathrm{MHz}, \mathrm{CDCl}_{3}\right): \delta 167.7,165.5,115.8,110.3$, 100.9, 74.6, 68.2, 67.8, 44.9, 35.0, 33.7, 32.4, 28.5, 25.7, 21.2, 20.8, 20.1. HRMS (ESI-TOF) $\mathrm{m} / \mathrm{z}:[\mathrm{M}+\mathrm{Na}]^{+}$Calcd for $\mathrm{C}_{17} \mathrm{H}_{21} \mathrm{NO}_{5} \mathrm{Na}$ 342.1312; Found 342.1297.
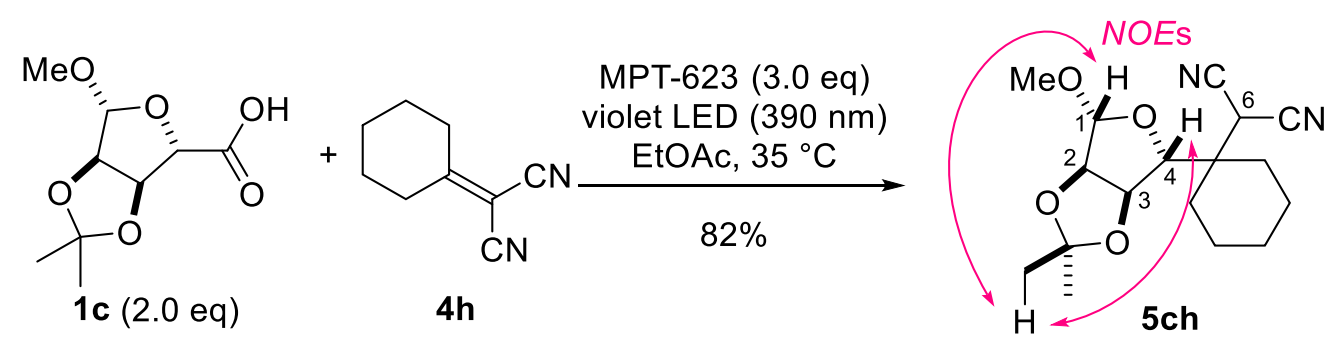

General procedure D: Compound 5ch. Argon was bubbled through a suspension of 1c (53.7 $\mathrm{mg}, 246 \mu \mathrm{mol})$, cyclohexylidenemalononitrile $4 \mathbf{h}(18.0 \mathrm{mg}, 123 \mu \mathrm{mol})$, and MPT-623 (29.5 mg, $369 \mu \mathrm{mol})$ in EtOAc $(3.7 \mathrm{~mL})$ at $24^{\circ} \mathrm{C}$ for $20 \mathrm{~min}$. The resultant mixture was sonicated for 3 min, stirred, and then irradiated with a violet LED. After being irradiated with stirring at $35^{\circ} \mathrm{C}$ for $16 \mathrm{~h}$, the resultant mixture was filtered and concentrated. The residue was purified by flash column chromatography on silica gel $\left(3 \mathrm{~g}\right.$, hexane $/ \mathrm{CH}_{2} \mathrm{Cl}_{2}=1 / 1$ to $1 / 2 ; 3 \mathrm{~g}$, hexane/EtOAc $=$ $15 / 1$ to $5 / 1)$ to afford $\mathbf{5 c h}(32.5 \mathrm{mg}, 101 \mu \mathrm{mol})$ in $82 \%$ yield. The C4-stereochemistry of $\mathbf{5} \mathbf{c h}$ was determined by the NOESY experiment (Page S64): colorless oil. $[\alpha]_{\mathrm{D}}^{26}-17.2$ (c 1.00, $\mathrm{CHCl}_{3}$ ). IR (film): 2936, 2253, 1456, 1379, 1212, 1158, 1086, 962, $864 \mathrm{~cm}^{-1} .{ }^{1} \mathrm{H}$ NMR (400 $\left.\mathrm{MHz}_{\mathrm{CDCl}}\right): \delta 5.04(1 \mathrm{H}, \mathrm{s}, \mathrm{H} 1), 4.88(1 \mathrm{H}, \mathrm{dd}, J=6.4,4.1 \mathrm{~Hz}, \mathrm{H} 3), 4.54(1 \mathrm{H}, \mathrm{d}, J=6.4 \mathrm{~Hz}$, H2), $4.38(1 \mathrm{H}, \mathrm{d}, J=4.1 \mathrm{~Hz}, \mathrm{H} 4), 4.18(1 \mathrm{H}, \mathrm{s}, \mathrm{H} 6), 3.47$ (3H, s, OCH $), 1.92(1 \mathrm{H}, \mathrm{m}), 1.77-$ $1.57(6 \mathrm{H}, \mathrm{m}), 1.54\left(3 \mathrm{H}, \mathrm{s}, \mathrm{CH}_{3}\right.$ of acetonide), 1.50-1.35 (3H, m), $1.34\left(3 \mathrm{H}, \mathrm{s}, \mathrm{CH}_{3}\right.$ of acetonide). ${ }^{13} \mathrm{C}\left\{{ }^{1} \mathrm{H}\right\}$ NMR $\left(100 \mathrm{MHz}, \mathrm{CDCl}_{3}\right): \delta 113.6,112.1,111.9,109.2,89.8,85.2,78.9,56.6,42.6$, 29.7, 28.3, 28.0, 27.0, 25.2, 24.9, 20.73, 20.69. HRMS (ESI-TOF) $\mathrm{m} / \mathrm{z}:[\mathrm{M}+\mathrm{Na}]^{+}$Calcd for $\mathrm{C}_{17} \mathrm{H}_{24} \mathrm{~N}_{2} \mathrm{O}_{4} \mathrm{Na} 343.1628$; Found 343.1628. 


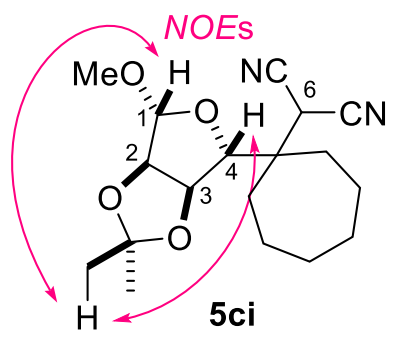

Compound 5ci. According to the general procedure D, 5ci $(20.2$ $\mathrm{mg}, 60.4 \mu \mathrm{mol})$ was synthesized in $60 \%$ yield from $1 \mathrm{c}(44.1 \mathrm{mg}, 202$ $\mu \mathrm{mol})$ and cycloheptylidenemalononitrile 4i [CAS: 10394-94-2] ] $10^{2}$ (16.2 mg, $101 \mu \mathrm{mol})$ by using MPT-623 (24.2 mg, $303 \mu \mathrm{mol})$ in EtOAc $(3.0 \mathrm{~mL})$. After being irradiated with stirring at $35^{\circ} \mathrm{C}$ for 13 $\mathrm{h}$, the resultant mixture was filtered and concentrated. The residue was purified by flash column chromatography on silica gel ( $3 \mathrm{~g}$, hexane/ $\mathrm{CH}_{2} \mathrm{Cl}_{2}=1 / 1$ to $1 / 2 ; 2$ $\mathrm{g}$, hexane/EtOAc $=12 / 1$ to $4 / 1)$. The C4-stereochemistry of $\mathbf{5 c i}$ was determined by the NOESY experiment (Page S66): colorless oil. $[\alpha]_{\mathrm{D}}{ }^{26}-25.7$ (c 1.01, $\mathrm{CHCl}_{3}$ ). IR (film): 2932, $2252,1461,1374,1214,1157,1084,981,862 \mathrm{~cm}^{-1} .{ }^{1} \mathrm{H}$ NMR $\left(400 \mathrm{MHz}, \mathrm{CDCl}_{3}\right): \delta 5.01(1 \mathrm{H}$, s, H1), $4.88(1 \mathrm{H}, \mathrm{dd}, J=6.4,4.1 \mathrm{~Hz}, \mathrm{H} 3), 4.54(1 \mathrm{H}, \mathrm{d}, J=6.4 \mathrm{~Hz}, \mathrm{H} 2), 4.15(1 \mathrm{H}, \mathrm{d}, J=4.1 \mathrm{~Hz}$, H4), $3.94(1 \mathrm{H}, \mathrm{s}, \mathrm{H} 6), 3.46\left(3 \mathrm{H}, \mathrm{s}, \mathrm{OCH}_{3}\right), 1.92-1.81(3 \mathrm{H}, \mathrm{m}), 1.77(1 \mathrm{H}, \mathrm{m}), 1.72-1.55(8 \mathrm{H}, \mathrm{m})$, $1.53\left(3 \mathrm{H}, \mathrm{s}, \mathrm{CH}_{3}\right.$ of acetonide), $1.33\left(3 \mathrm{H}, \mathrm{s}, \mathrm{CH}\right.$ of acetonide). ${ }^{13} \mathrm{C}\left\{{ }^{1} \mathrm{H}\right\}$ NMR $(100 \mathrm{MHz}$, $\left.\mathrm{CDCl}_{3}\right): \delta 113.8,112.7,112.5,108.9,91.1,85.1,78.6,56.5,45.8,34.3,31.8,30.9,30.5,30.2$, 27.0, 25.2, 23.8, 23.0. HRMS (ESI-TOF) m/z: $[\mathrm{M}+\mathrm{Na}]^{+}$Calcd for $\mathrm{C}_{18} \mathrm{H}_{26} \mathrm{~N}_{2} \mathrm{O}_{4} \mathrm{Na}$ 357.1785; Found 357.1775 .

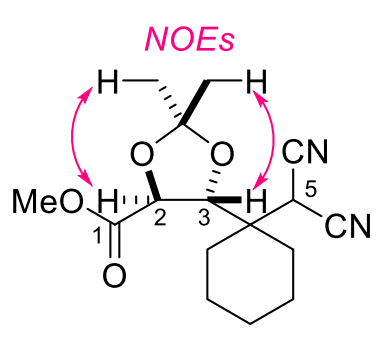

$5 \mathrm{fh}$

Compound 5fh. According to the general procedure D, 5fh (18.8 $\mathrm{mg}, 61.4 \mu \mathrm{mol})$ was synthesized in $56 \%$ yield from 1f $(44.2 \mathrm{mg}, 216$ $\mu \mathrm{mol})$ and $4 \mathrm{~h}(16.1 \mathrm{mg}, 110 \mu \mathrm{mol})$ by using MPT-623 (26.4 mg, 330 $\mu \mathrm{mol})$ in EtOAc $(3.3 \mathrm{~mL})$. After being irradiated with stirring at $35{ }^{\circ} \mathrm{C}$ for $13 \mathrm{~h}$, the resultant mixture was filtered and concentrated. The residue was purified by flash column chromatography on silica gel $(4 \mathrm{~g}$, hexane/EtOAc $=6 / 1)$ and PTLC $(20 \mathrm{~cm} \times 10 \mathrm{~cm}$, hexane/acetone $=10 / 1$ ). The C3-stereochemistry of $\mathbf{5} \mathbf{f h}$ was determined by the NOESY experiment (Page S68): colorless oil. $[\alpha]_{\mathrm{D}}{ }^{21}+18.8$ (c 1.04, $\mathrm{CHCl}_{3}$ ). IR (film): 2941, 2323, 1750, 1450, 1380, 1214, $1098 \mathrm{~cm}^{-1}$. ${ }^{1} \mathrm{H}$ NMR $\left(400 \mathrm{MHz}, \mathrm{CDCl}_{3}\right): \delta 4.61(1 \mathrm{H}, \mathrm{d}, J=6.4 \mathrm{~Hz}, \mathrm{H} 2), 4.58(1 \mathrm{H}, \mathrm{d}, J=6.4$ $\mathrm{Hz}, \mathrm{H} 3), 4.15(1 \mathrm{H}, \mathrm{s}, \mathrm{H} 5), 3.82\left(3 \mathrm{H}, \mathrm{s}, \mathrm{CO}_{2} \mathrm{CH}_{3}\right), 1.91(1 \mathrm{H}, \mathrm{m}), 1.77-1.57(6 \mathrm{H}, \mathrm{m}), 1.54(3 \mathrm{H}, \mathrm{s}$, $\mathrm{CH}_{3}$ of acetonide), $1.50-1.33(3 \mathrm{H}, \mathrm{m}), 1.42\left(3 \mathrm{H}, \mathrm{s}, \mathrm{CH}_{3}\right.$ of acetonide). ${ }^{13} \mathrm{C}\left\{{ }^{1} \mathrm{H}\right\} \mathrm{NMR}(100 \mathrm{MHz}$, $\left.\mathrm{CDCl}_{3}\right): \delta 171.7,112.0,111.70,111.68,81.4,74.4,52.8,42.4,30.1,28.3,28.0,26.2,25.1,24.8$, 20.8, 20.7. HRMS (ESI-TOF) $\mathrm{m} / \mathrm{z}$ : $[\mathrm{M}+\mathrm{Na}]^{+}$Calcd for $\mathrm{C}_{16} \mathrm{H}_{22} \mathrm{~N}_{2} \mathrm{O}_{4} \mathrm{Na}$ 329.1472; Found 329.1457 . 


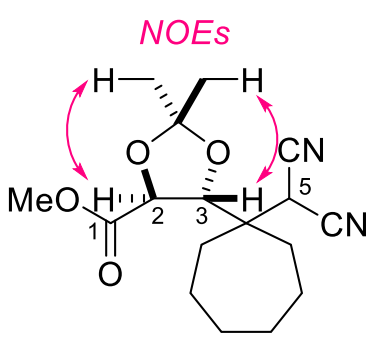

$5 \mathrm{fi}$

Compound 5fi. According to the general procedure D, 5fi (10.3 mg, $32.1 \mu \mathrm{mol})$ was synthesized in $31 \%$ yield from $\mathbf{1 f}(42.1 \mathrm{mg}, 206 \mu \mathrm{mol})$ and $4 \mathbf{i}(16.5 \mathrm{mg}, 103 \mu \mathrm{mol})$ by using MPT-623 (24.8 mg, $310 \mu \mathrm{mol})$ in EtOAc $(3.1 \mathrm{~mL})$. After being irradiated with stirring at $35^{\circ} \mathrm{C}$ for 13 $\mathrm{h}$, the resultant mixture was filtered and concentrated. The residue was purified by flash column chromatography on silica gel (4 g, hexane/EtOAc $=6 / 1)$ and PTLC $(20 \mathrm{~cm} \times 10 \mathrm{~cm}$, hexane/acetone $=$

10/1) to afford $\mathbf{5 f i}$. The C3-stereochemistry of $\mathbf{5 f i}$ was determined by the NOESY experiment (Page S70): colorless oil. $[\alpha]_{\mathrm{D}}{ }^{21}+13.2\left(c 0.86, \mathrm{CHCl}_{3}\right)$. IR (film): 2930, 2322, 1749, 1452, 1380, $1213,1088 \mathrm{~cm}^{-1} .{ }^{1} \mathrm{H}$ NMR (400 MHz, $\left.\mathrm{CDCl}_{3}\right): \delta 4.58(1 \mathrm{H}, \mathrm{d}, J=6.4 \mathrm{~Hz}, \mathrm{H} 2), 4.42(1 \mathrm{H}, \mathrm{d}, J=$ $6.9 \mathrm{~Hz}, \mathrm{H} 3), 3.93$ (1H, s, H5), $3.83\left(3 \mathrm{H}, \mathrm{s}, \mathrm{CO}_{2} \mathrm{CH}_{3}\right), 1.94-1.88(2 \mathrm{H}, \mathrm{m}), 1.81-1.73(2 \mathrm{H}, \mathrm{m})$, 1.73-1.50 (8H, m), $1.54\left(3 \mathrm{H}, \mathrm{s}, \mathrm{CH}_{3}\right.$ of acetonide), $1.40\left(3 \mathrm{H}, \mathrm{s}, \mathrm{CH}_{3}\right.$ of acetonide). ${ }^{13} \mathrm{C}\left\{{ }^{1} \mathrm{H}\right\}$ $\operatorname{NMR}\left(100 \mathrm{MHz}, \mathrm{CDCl}_{3}\right): \delta 171.7,112.4,112.2,111.5,82.7,74.5,52.9,45.5,34.3,32.4,30.9$, 30.8, 30.5, 26.3, 25.0, 24.0, 23.2. HRMS (ESI-TOF) $\mathrm{m} / \mathrm{z}$ : $[\mathrm{M}+\mathrm{Na}]^{+}$Calcd for $\mathrm{C}_{17} \mathrm{H}_{24} \mathrm{~N}_{2} \mathrm{O}_{4} \mathrm{Na}$ 343.1628; Found 343.1616.

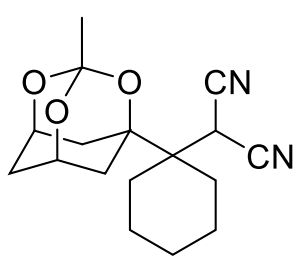

$5 \mathrm{gh}$

Compound 5gh [CAS: 1326319-43-0]. According to the general procedure D, 5gh $(23.6 \mathrm{mg}, 78.1 \mu \mathrm{mol})$ was synthesized in $74 \%$ yield from $1 \mathrm{~g}(42.1 \mathrm{mg}, 210 \mu \mathrm{mol})$ and $\mathbf{4 h}(15.4 \mathrm{mg}, 105 \mu \mathrm{mol})$ by using MPT-623 $(25.2 \mathrm{mg}, 315 \mu \mathrm{mol})$ in EtOAc $(3.2 \mathrm{~mL})$. After being irradiated with stirring at $35{ }^{\circ} \mathrm{C}$ for $16 \mathrm{~h}$, the resultant mixture was filtered and concentrated. The residue was purified by flash column chromatography on silica gel $(4 \mathrm{~g}$, hexane/EtOAc $=6 / 1)$ and PTLC $(20 \mathrm{~cm} \times 10 \mathrm{~cm}$, hexane/acetone $=10 / 1)$. The analytical data of $\mathbf{5 g h}$ was identical to those reported previously. ${ }^{\mathrm{S} 9}$

${ }^{1} \mathrm{H}$ NMR (400 MHz, $\left.\mathrm{CDCl}_{3}\right): \delta 4.48(2 \mathrm{H}, \mathrm{m}), 4.29(1 \mathrm{H}, \mathrm{s}), 2.55(3 \mathrm{H}, \mathrm{m}), 1.91(2 \mathrm{H}, \mathrm{m}), 1.82-$ $1.74(5 \mathrm{H}, \mathrm{m}), 1.69-1.60(3 \mathrm{H}, \mathrm{m}), 1.46(3 \mathrm{H}, \mathrm{s}), 1.37-1.14(3 \mathrm{H}, \mathrm{m})$.

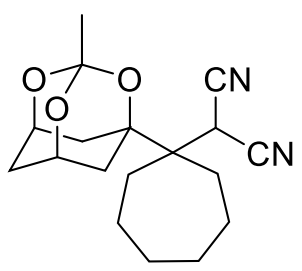

$5 \mathrm{gi}$

Compound 5gi [CAS: 1326319-44-1]. According to the general procedure D, 5gi $(27.6 \mathrm{mg}, 87.2 \mu \mathrm{mol})$ was synthesized in $84 \%$ yield from $1 \mathrm{~g}(41.7 \mathrm{mg}, 208 \mu \mathrm{mol})$ and $4 \mathbf{i}(16.7 \mathrm{mg}, 104 \mu \mathrm{mol})$ by using MPT-623 $(25.0 \mathrm{mg}, 312 \mu \mathrm{mol})$ in EtOAc $(3.1 \mathrm{~mL})$. After being irradiated with stirring at $35{ }^{\circ} \mathrm{C}$ for $18 \mathrm{~h}$, the resultant mixture was filtered and concentrated. The residue was purified by flash column chromatography on silica gel ( $3 \mathrm{~g}$, hexane/EtOAc $=5 / 1$ to $2 / 1)$. The analytical data of 5gi was identical to those reported previously. ${ }^{\mathrm{S} 9}$

${ }^{1} \mathrm{H}$ NMR (400 MHz, $\left.\mathrm{CDCl}_{3}\right): \delta, 4.46(2 \mathrm{H}, \mathrm{m}), 4.11(1 \mathrm{H}, \mathrm{s}), 2.55-2.48(3 \mathrm{H}, \mathrm{m}), 2.04-1.98(2 \mathrm{H}$, m), 1.78-1.56 (13H, m), $1.47(3 \mathrm{H}, \mathrm{s})$. 


\section{Hydrogen transfer experiments}

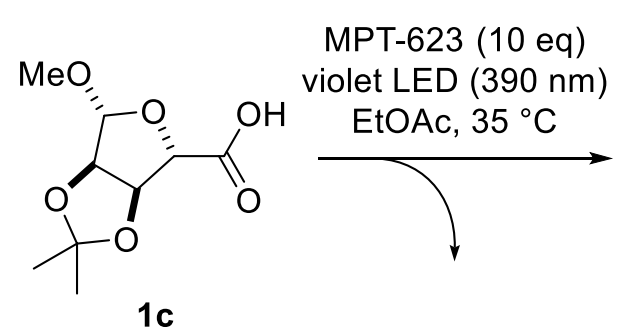

1c

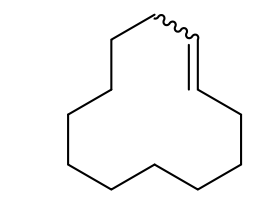

14 (cyclododecene)

$(E / Z=1: 1.7)$

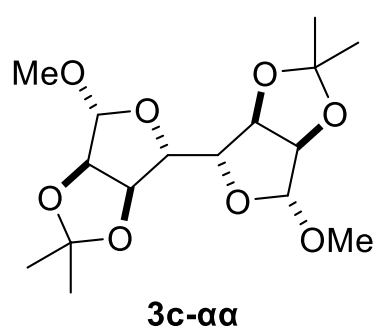

$31 \%$

(NMR yield)

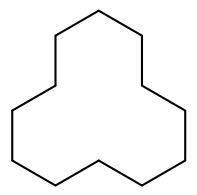

15 (cyclododecane)

$71 \%$

(NMR yield)<smiles>CO[C@H]1OC(C2O[C@H](OC)[C@H]3OC(C)(C)O[C@H]23)C2OC(C)(C)OC21</smiles>

$31 \%$

(NMR yield)

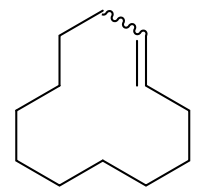

14

$4 \%(E / Z=1: 1)$

(NMR yield)

In chamber A of a screw-capped two-chamber glass system (COware), 1c (21.8 mg, $100 \mu \mathrm{mol})$, and MPT-623 (80.0 mg, $1.00 \mathrm{mmol})$ were dissolved in EtOAc $(1.0 \mathrm{~mL})$, and argon was bubbled through the solution at $24{ }^{\circ} \mathrm{C}$ for $20 \mathrm{~min}$. In chamber B of the apparatus, cyclododecene 14 $(19.1 \mu \mathrm{L}, 99.9 \mu \mathrm{mol})$ and $\mathrm{RhCl}\left(\mathrm{PPh}_{3}\right)_{3}(18.5 \mathrm{mg}, 20.0 \mu \mathrm{mol})$ were dissolved in EtOAc $(1.0 \mathrm{~mL})$, and argon was bubbled through the solution at $24{ }^{\circ} \mathrm{C}$ for $20 \mathrm{~min}$. The apparatus was sonicated for $3 \mathrm{~min}$, and chamber B was wrapped with aluminum foil. The reaction mixture in chamber A was stirred and irradiated with a violet LED at $35{ }^{\circ} \mathrm{C}$ for $16 \mathrm{~h}$. The reaction mixtures in both chambers were stirred for $51 \mathrm{~h}$ at $24{ }^{\circ} \mathrm{C}$ without photo-irradiation. The reaction mixture

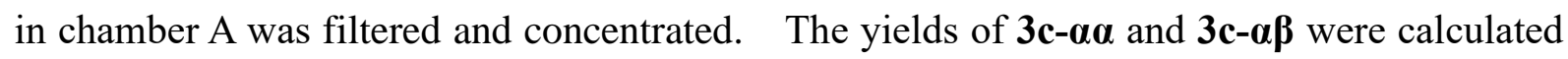
to be $31 \%(31.3 \mu \mathrm{mol})$ and $31 \%(31.3 \mu \mathrm{mol})$, respectively, by ${ }^{1} \mathrm{H}$ NMR analysis using $p$-xylene $(10.4 \mathrm{mg}, 98.0 \mu \mathrm{mol})$ as the internal standard. The combined NMR yield of two diastereomers 3c was $62 \%$, and the $\alpha \alpha / \alpha \beta$ ratio was 50:50. The reaction mixture in chamber B was passed through a pad of silica gel with $\mathrm{CH}_{2} \mathrm{Cl}_{2}$, and then concentrated. The yield of $\mathbf{1 5}$ and the remained 14 was calculated to be $71 \%(71.3 \mu \mathrm{mol})$ and $4 \%(3.88 \mu \mathrm{mol}, E / Z=1: 1)$, respectively, by ${ }^{1} \mathrm{H}$ NMR analysis using $p$-xylene $(10.3 \mathrm{mg}, 97.0 \mu \mathrm{mol})$ as the internal standard. 


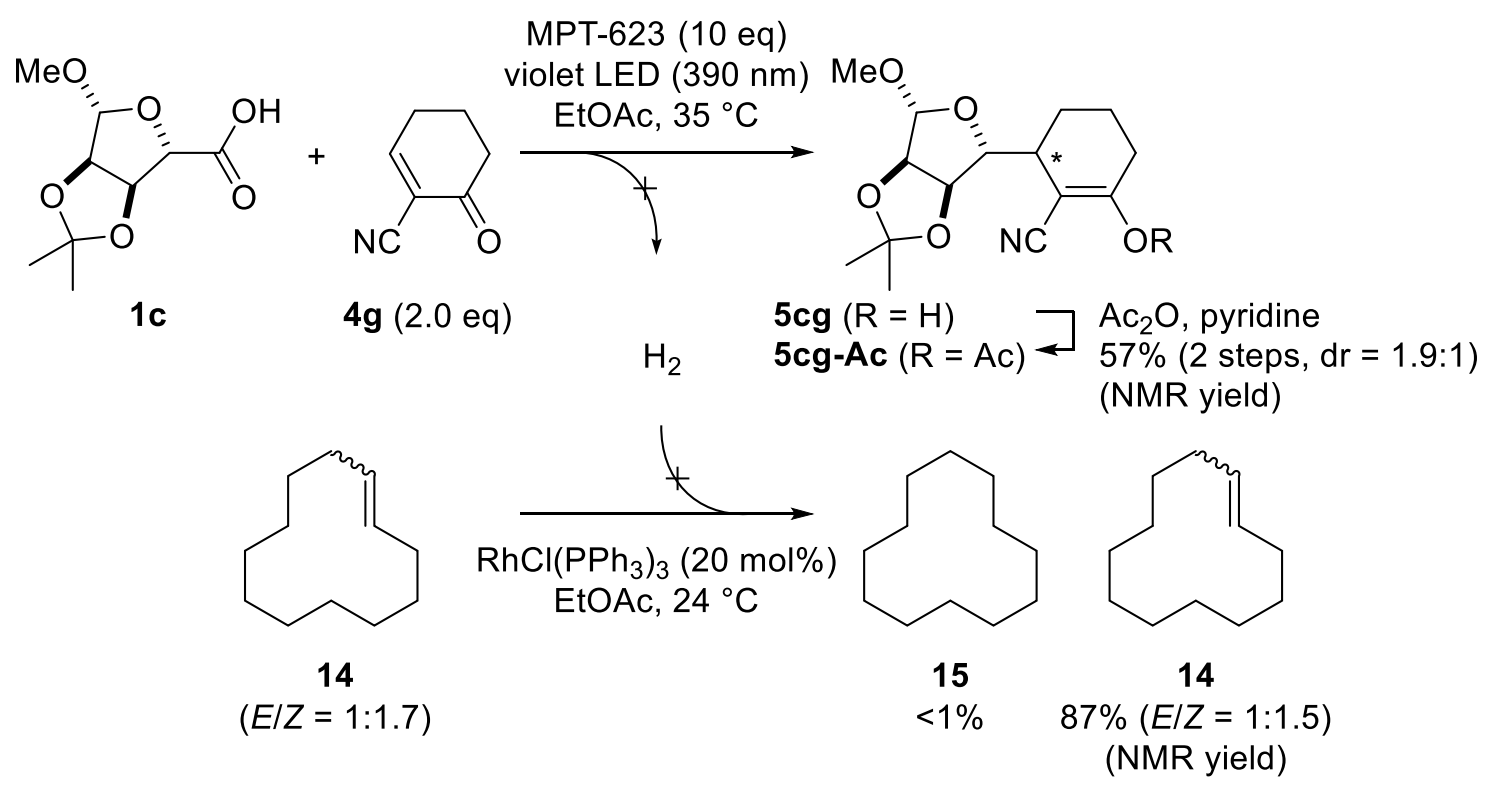

In chamber A of a screw-capped two-chamber glass system (COware), 1c (21.8 mg, 0.100 $\mathrm{mmol}), 4 \mathrm{~g}$ (24.2 $\mathrm{mg}, 0.200 \mathrm{mmol})$, and MPT-623 (12.0 $\mathrm{mg}, 0.150 \mathrm{mmol})$ were dissolved in EtOAc $(3.0 \mathrm{~mL})$, and argon was bubbled through the solution at $24{ }^{\circ} \mathrm{C}$ for $20 \mathrm{~min}$. In chamber $\mathrm{B}$ of the apparatus, cyclododecene $14(19.1 \mu \mathrm{L}, 99.9 \mu \mathrm{mol})$ and $\mathrm{RhCl}\left(\mathrm{PPh}_{3}\right)_{3}(18.5 \mathrm{mg}, 20.0$ $\mu \mathrm{mol})$ were dissolved in degassed EtOAc $(1.0 \mathrm{~mL})$. The apparatus was sonicated for $3 \mathrm{~min}$, and chamber B was wrapped with aluminum foil. The reaction mixture in chamber A was stirred and irradiated with a violet LED at $35{ }^{\circ} \mathrm{C}$ for $8 \mathrm{~h}$. The reaction mixtures in both chambers were stirred for $64 \mathrm{~h}$ at $24{ }^{\circ} \mathrm{C}$ without photo-irradiation. The reaction mixture in chamber A was filtered and concentrated. The residue was purified by flash column chromatography on silica gel ( 3 g, hexane/EtOAc $=5 / 1$ to $4 / 1$ to $3 / 1)$ to afford crude $\mathbf{5 c g}(30.7$ $\mathrm{mg}$ ), which was used in the next reaction without further purification. The reaction mixture in chamber B was passed through a pad of silica gel with $\mathrm{CH}_{2} \mathrm{Cl}_{2}$, and then concentrated. The yield of remained 14 was calculated to be $87 \%$ ( $86.5 \mu \mathrm{mol}, E / Z=1: 1.5)$, by ${ }^{1} \mathrm{H}$ NMR analysis using $p$-xylene $(10.2 \mathrm{mg}, 96.1 \mu \mathrm{mol})$ as the internal standard.

The above crude $5 \mathbf{c g}$ was dissolved in $\mathrm{CH}_{2} \mathrm{Cl}_{2}(1.5 \mathrm{~mL})$, pyridine $(0.60 \mathrm{~mL})$ and $\mathrm{Ac}_{2} \mathrm{O}(0.30$ $\mathrm{mL}$ ) at $29^{\circ} \mathrm{C}$. After the reaction mixture was stirred at $29^{\circ} \mathrm{C}$ for $2 \mathrm{~h}$, the resultant mixture was concentrated. The residue was passed through a pad of silica gel (hexane/EtOAc $=5 / 1$ ). The combined fractions were washed with $\mathrm{H}_{2} \mathrm{O}\left(10 \mathrm{~mL}\right.$ x2), dried over $\mathrm{Na}_{2} \mathrm{SO}_{4}$, filtered, and concentrated. The yield of 5cg-Ac was calculated to be $57 \%(56.7 \mu \mathrm{mol})$ over 2 steps, by ${ }^{1} \mathrm{H}$ NMR analysis using $p$-xylene $(10.9 \mathrm{mg}, 103 \mu \mathrm{mol})$ as the internal standard. The diastereomeric ratio of two diastereomers $\mathbf{5} \mathbf{c g}$-Ac was 1.9:1. 


\section{MPT-623 recycling experiments}

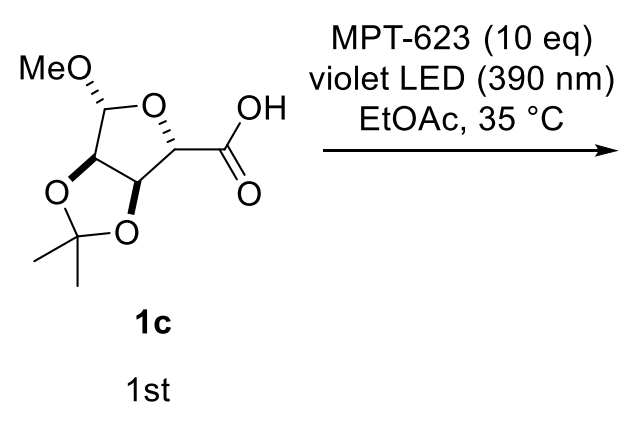

2nd

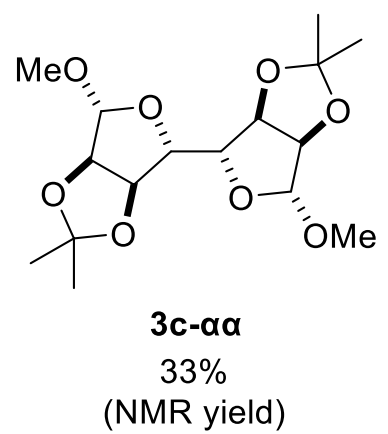

$33 \%$

(NMR yield)

$34 \%$

(NMR yield)

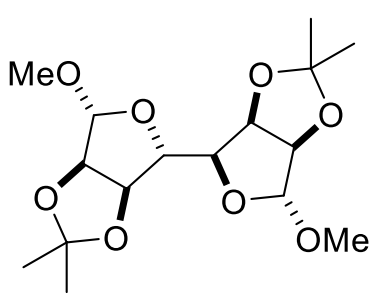

$3 c-\alpha \beta$

$34 \%$

(NMR yield)

$33 \%$

(NMR yield)

$34 \%$

(NMR yield)

Dimer 3c. According to the general procedure A, a diastereomeric mixture of $\mathbf{3 c - \alpha \boldsymbol { \alpha }}$ and $\mathbf{3 c}$ $\boldsymbol{\alpha} \boldsymbol{\beta}$ was synthesized from carboxylic acid $1 \mathrm{c}(43.6 \mathrm{mg}, 200 \mu \mathrm{mol})$ by using MPT-623 (160 mg, $2.00 \mathrm{mmol})$ in EtOAc $(2.0 \mathrm{~mL})$. After being irradiated with stirring at $35{ }^{\circ} \mathrm{C}$ for $16 \mathrm{~h}$, the resultant mixture was filtered using a Kiriyama funnel fitted with No. $5 \mathrm{C}$ filter paper using $\mathrm{MeOH}(10 \mathrm{~mL})$ and EtOAc $(3 \mathrm{~mL})$ to recover MPT-623 $(155 \mathrm{mg})$ in $97 \%$ yield, which was used in the next reaction without further purification. The filtrate was further filtered using a membrane filter with $\mathrm{MeOH}(5 \mathrm{~mL})$ and concentrated. The yields of $\mathbf{3 c}-\boldsymbol{\alpha \alpha}$ and $\mathbf{3 c}-\boldsymbol{\alpha} \boldsymbol{\beta}$ were calculated to be $33 \%(32.7 \mu \mathrm{mol})$ and $34 \%(34.3 \mu \mathrm{mol})$, respectively, by ${ }^{1} \mathrm{H}$ NMR analysis using $p$-xylene $(10.6 \mathrm{mg}, 100 \mu \mathrm{mol})$ as the internal standard. The combined NMR yield of two diastereomers $3 \mathbf{c}$ was $67 \%$, and the $\alpha \alpha / \alpha \beta$ ratio was $49: 51$.

According to the general procedure A, a diastereomeric mixture of $\mathbf{3 c - \alpha \boldsymbol { \alpha }}$ and $\mathbf{3 c - \alpha \boldsymbol { \beta }}$ was synthesized from 1c $(42.3 \mathrm{mg}, 194 \mu \mathrm{mol})$ by using the above-recovered MPT-623 (155 mg, $1.94 \mathrm{mmol})$ in EtOAc $(1.9 \mathrm{~mL})$. After being irradiated with stirring at $35{ }^{\circ} \mathrm{C}$ for $16 \mathrm{~h}$, the resultant mixture was filtered using a Kiriyama funnel fitted with No. $5 \mathrm{C}$ filter paper using $\mathrm{MeOH}(10 \mathrm{~mL})$ and EtOAc $(3 \mathrm{~mL})$ to recover MPT-623 $(147 \mathrm{mg})$ in 95\% yield, which was used in the next reaction without further purification. The filtrate was further filtered using a membrane filter with $\mathrm{MeOH}(5 \mathrm{~mL})$ and concentrated. The yields of $\mathbf{3 c}-\boldsymbol{\alpha \alpha}$ and $\mathbf{3 c}-\boldsymbol{\alpha} \boldsymbol{\beta}$ were calculated to be $33 \%(32.3 \mu \mathrm{mol})$ and $33 \%(32.3 \mu \mathrm{mol})$, respectively, by ${ }^{1} \mathrm{H}$ NMR analysis using $p$-xylene $(10.4 \mathrm{mg}, 98.0 \mu \mathrm{mol})$ as the internal standard. The combined NMR yield of two diastereomers $3 \mathrm{c}$ was $66 \%$, and the $\alpha \alpha / \alpha \beta$ was $50: 50$.

According to the general procedure A, a diastereomeric mixture of $\mathbf{3 c - \alpha \boldsymbol { \alpha }}$ and $\mathbf{3 c - \alpha} \boldsymbol{\beta}$ was synthesized from 1c $(40.1 \mathrm{mg}, 184 \mu \mathrm{mol})$ by using the above-recovered MPT-623 (147 mg, $1.84 \mathrm{mmol})$ in EtOAc $(1.8 \mathrm{~mL})$. After being irradiated with stirring at $35^{\circ} \mathrm{C}$ for $16 \mathrm{~h}$, the resultant mixture was filtered using a Kiriyama funnel fitted with No. $5 \mathrm{C}$ filter paper using $\mathrm{MeOH}(10 \mathrm{~mL})$ and EtOAc $(3 \mathrm{~mL})$ to recover MPT-623 $(142 \mathrm{mg})$ in 97\% yield. The filtrate 
was further filtered using a membrane filter with $\mathrm{MeOH}(5 \mathrm{~mL})$ and concentrated. The yields of $\mathbf{3 c - \alpha \alpha}$ and $\mathbf{3 c - \alpha \boldsymbol { \beta }}$ were calculated to be $34 \%$ (31.7 $\mu \mathrm{mol})$ and 34\% (31.7 $\mu \mathrm{mol})$, respectively, by ${ }^{1} \mathrm{H}$ NMR analysis using $p$-xylene $(10.5 \mathrm{mg}, 98.9 \mathrm{mmol})$ as the internal standard. The combined NMR yield of two diastereomers $3 \mathbf{c}$ was $68 \%$, and the $\alpha \alpha / \alpha \beta$ ratio was 50:50.

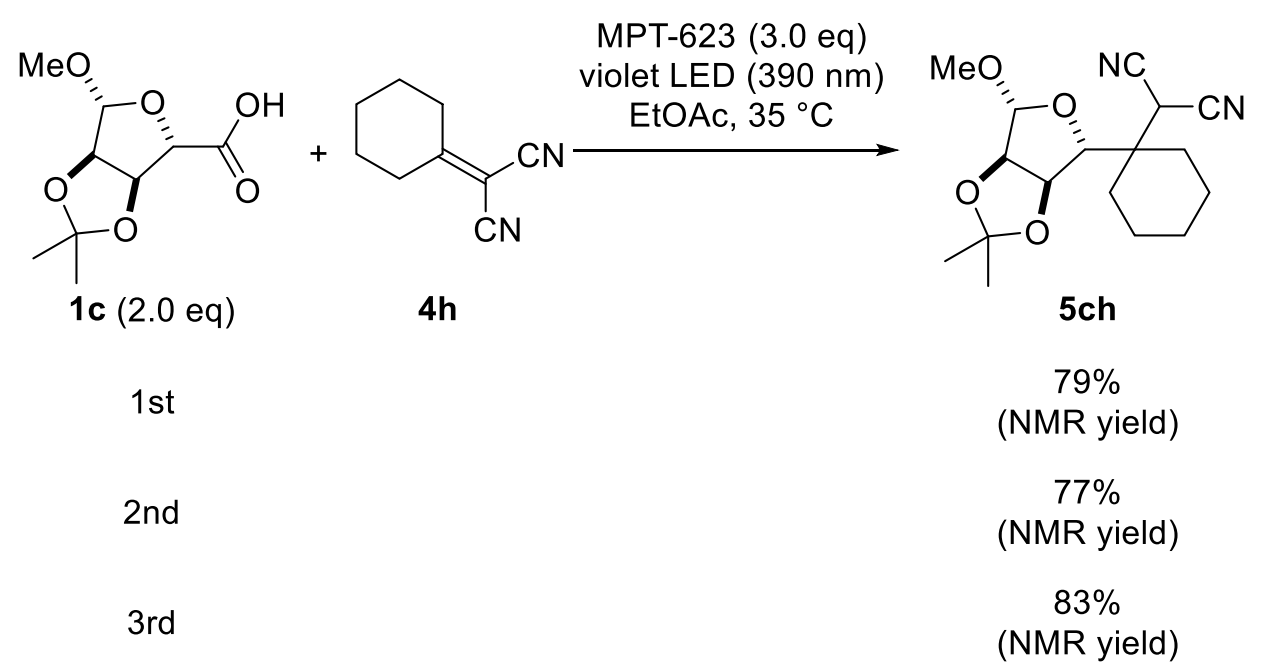

Compound 5ch. According to the general procedure D, 5ch was synthesized from 1c (59.1 $\mathrm{mg}, 271 \mu \mathrm{mol})$ and $\mathbf{4 h}(19.8 \mathrm{mg}, 135 \mu \mathrm{mol})$ by using MPT-623 (32.5 mg, $406 \mu \mathrm{mol})$ in EtOAc (4.1 mL). After being irradiated with stirring at $35{ }^{\circ} \mathrm{C}$ for $17 \mathrm{~h}$, the resultant mixture was filtered using a Kiriyama funnel fitted with No. $5 \mathrm{C}$ filter paper using $\mathrm{MeOH}(10 \mathrm{~mL})$ and EtOAc $(3 \mathrm{~mL})$ to recover MPT-623 $(26.4 \mathrm{mg})$ in $81 \%$ yield, which was used in the next reaction without further purification. The filtrate was further filtered using a membrane filter with $\mathrm{MeOH}(5$ $\mathrm{mL})$ and concentrated. The yield of $\mathbf{5} \mathbf{c h}$ was calculated to be $79 \%(107 \mu \mathrm{mol})$ by ${ }^{1} \mathrm{H} \mathrm{NMR}$ analysis using $p$-xylene $(10.6 \mathrm{mg}, 100 \mu \mathrm{mol})$ as the internal standard.

According to the general procedure D, 5ch was synthesized from 1c $(48.0 \mathrm{mg}, 220 \mu \mathrm{mol})$ and 4h $(16.1 \mathrm{mg}, 110 \mu \mathrm{mol})$ by using the above-recovered MPT-623 $(26.4 \mathrm{mg}, 330 \mu \mathrm{mol})$ in EtOAc $(3.3 \mathrm{~mL})$. After being irradiated with stirring at $35^{\circ} \mathrm{C}$ for $17 \mathrm{~h}$, the resultant mixture was filtered using a Kiriyama funnel fitted with No. $5 \mathrm{C}$ filter paper using $\mathrm{MeOH}(10 \mathrm{~mL})$ and EtOAc (3 mL) to recover MPT-623 $(20.6 \mathrm{mg})$ in $78 \%$ yield, which was used in the next reaction without further purification. The filtrate was further filtered using a membrane filter with $\mathrm{MeOH}(5$ $\mathrm{mL})$ and concentrated. The yield of $\mathbf{5} \mathbf{c h}$ was calculated to be $77 \%(84.3 \mu \mathrm{mol})$ by ${ }^{1} \mathrm{H} \mathrm{NMR}$ analysis using $p$-xylene $(10.4 \mathrm{mg}, 98.0 \mu \mathrm{mol})$ as the internal standard.

According to the general procedure D, 5ch was synthesized from 1c $(37.5 \mathrm{mg}, 172 \mu \mathrm{mol})$ and 4h $(12.6 \mathrm{mg}, 86.2 \mu \mathrm{mol})$ by using the above-recovered MPT-623 $(20.6 \mathrm{mg}, 258 \mu \mathrm{mol})$ in EtOAc $(2.6 \mathrm{~mL})$. After being irradiated with stirring at $35^{\circ} \mathrm{C}$ for $18 \mathrm{~h}$, the resultant mixture was filtered using a Kiriyama funnel fitted with No. $5 \mathrm{C}$ filter paper using $\mathrm{MeOH}(10 \mathrm{~mL})$ and EtOAc (3 mL) to recover MPT-623 (15.0 mg) in 73\% yield. The filtrate was further filtered using a 
membrane filter with $\mathrm{MeOH}(5 \mathrm{~mL})$ and concentrated. The yield of $\mathbf{5} \mathbf{c h}$ was calculated to be $83 \%(71.2 \mu \mathrm{mol})$ by ${ }^{1} \mathrm{H}$ NMR analysis using $p$-xylene $(10.5 \mathrm{mg}, 98.9 \mu \mathrm{mol})$ as the internal standard.

\section{Experiments on $1 \mathrm{mmol}$ scale}
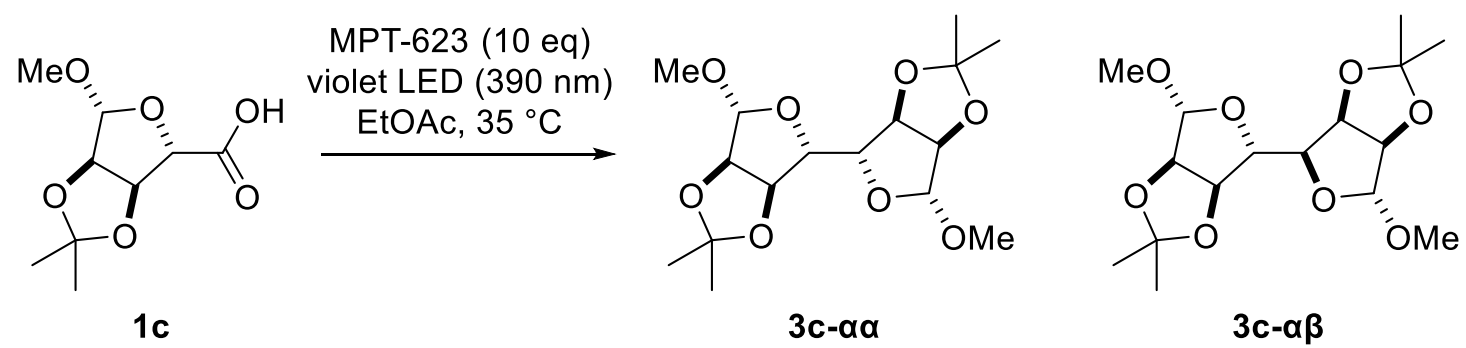

Dimer 3c. According to the general procedure A, 3c- $\boldsymbol{\alpha \alpha}(59.2 \mathrm{mg}, 171 \mu \mathrm{mol})$ and $\mathbf{3 c}-\boldsymbol{\alpha} \boldsymbol{\beta}(61.5$ $\mathrm{mg}, 177 \mu \mathrm{mol})$ were synthesized from carboxylic acid $1 \mathrm{c}(218 \mathrm{mg}, 1.00 \mathrm{mmol})$ by using MPT$623(800 \mathrm{mg}, 10.0 \mathrm{mmol})$ in EtOAc $(10.0 \mathrm{~mL})$ and a $30 \mathrm{~mL}$ round-bottom flask. After being irradiated with stirring at $35{ }^{\circ} \mathrm{C}$ for $16 \mathrm{~h}$, the resultant mixture was filtered with a pad of Celite $(3.6 \mathrm{~g})$ with EtOAc $(30 \mathrm{~mL})$ and concentrated. The residue was purified by automated flash column chromatography using the prepacked silica gel $(10 \mathrm{~g}$, hexane/EtOAc $=92 / 8$ to $72 / 28$ to $65 / 35 ; 10 \mathrm{~g}$, hexane/EtOAc $=88 / 12$ to $67 / 33$ ). The combined yield of two diastereomers $3 \mathbf{c}$ was $69 \%$, and the $\alpha \alpha / \alpha \beta$ ratio was $49: 51$.
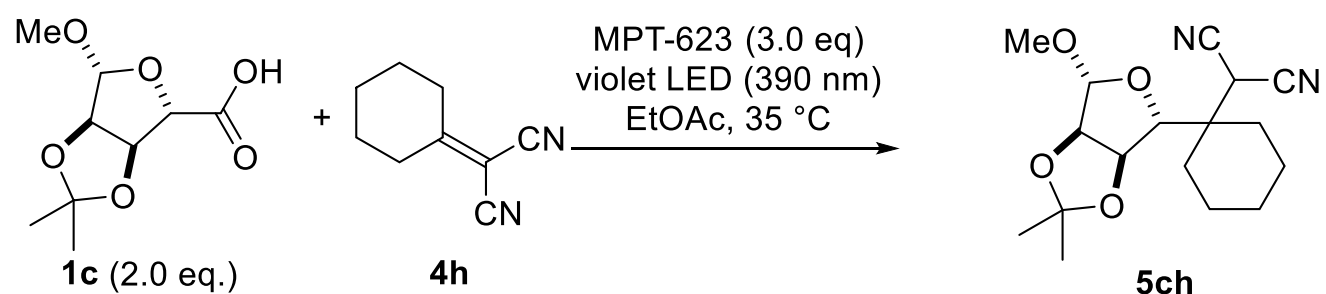

Compound 5ch. According to the general procedure D, 5ch (110 mg, $34.3 \mu \mathrm{mol})$ was synthesized in $68 \%$ yield from $1 \mathrm{c}(219 \mathrm{mg}, 1.00 \mathrm{mmol})$ and $\mathbf{4 h}(73.4 \mathrm{mg}, 502 \mu \mathrm{mol})$ by using MPT-623 $(120 \mathrm{mg}, 1.50 \mathrm{mmol})$ in EtOAc $(16.7 \mathrm{~mL})$ and a $50 \mathrm{~mL}$ round-bottom flask. After being irradiated with stirring at $35^{\circ} \mathrm{C}$ for $16 \mathrm{~h}$, the resultant mixture was filtered with a pad of Celite $(3.6 \mathrm{~g})$ with EtOAc $(30 \mathrm{~mL})$. The filtrate was further filtered using a membrane filter with EtOAc $(20 \mathrm{~mL})$ and concentrated. The residue was purified by flash column chromatography on silica gel $\left(10 \mathrm{~g}\right.$, hexane $/ \mathrm{CH}_{2} \mathrm{Cl}_{2}=1 / 1$ to $1 / 2 ; 10 \mathrm{~g}$, hexane/EtOAc $=15 / 1$ to $4 / 1)$. 


\section{Investigation of the light source}

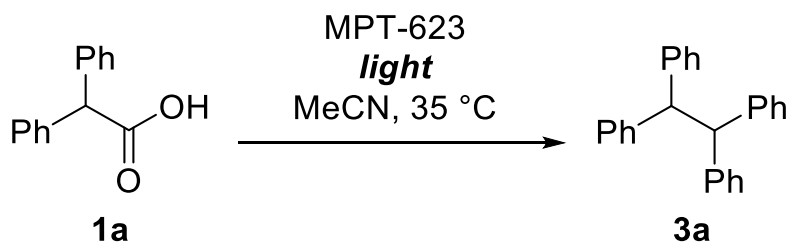

Table S1. Investigation of the light source ${ }^{a}$

\begin{tabular}{clcc}
\hline & & \multicolumn{2}{c}{ yield $(\%)^{b}$} \\
\cline { 3 - 4 } entry & & 3a & 1a \\
\hline 1 & LED of Shenzhen Chuwo Technology $(385-400 \mathrm{~nm})$ & 41 & 54 \\
2 & Xe-lamp (410 nm) & 13 & 56 \\
3 & LED of Techno Sigma $(385 \mathrm{~nm})$ & 22 & 53 \\
4 & Kessil LED $(370 \mathrm{~nm})$ & 56 & 0 \\
5 & Kessil LED $(390 \mathrm{~nm})$ & 88 & 0
\end{tabular}

${ }^{a}$ Reagents and conditions: 1a $\left(0.2 \mathrm{mmol}, 1\right.$ equiv), MPT-623 (10 equiv), light, $\mathrm{MeCN}(0.1 \mathrm{M}), 35{ }^{\circ} \mathrm{C}$, 3-16 $\mathrm{h}$. The reaction mixture was cooled with a circulator.

${ }^{b}$ Yields were determined by ${ }^{1} \mathrm{H}$ NMR analysis using $p$-xylene as the internal standard. 


\section{Attempted Ir-catalyzed dimerization and radical addition}

Scheme S1. Attempted Ir-catalyzed dimerization and radical addition ${ }^{\text {S11 }}$

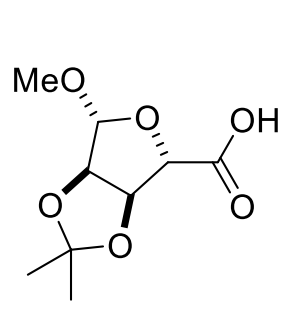

$1 \mathrm{c}$
$\operatorname{Ir}\left[\mathrm{dF}\left(\mathrm{CF}_{3}\right) \mathrm{ppy}\right]_{2}(\mathrm{dtbbpy}) \mathrm{PF}_{6}(2.5 \mathrm{~mol} \%)$ $\mathrm{K}_{2} \mathrm{HPO}_{4}(1.5 \mathrm{eq})$ blue LED $(448 \mathrm{~nm})$ DMF, $25^{\circ} \mathrm{C}$

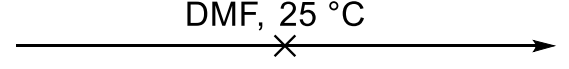

$\operatorname{Ir}\left[\mathrm{dF}\left(\mathrm{CF}_{3}\right) \mathrm{ppy}\right]_{2}(\mathrm{dtbbpy}) \mathrm{PF}_{6}(2.5 \mathrm{~mol} \%)$ $\mathrm{K}_{2} \mathrm{HPO}_{4}(1.5 \mathrm{eq})$ blue LED $(448 \mathrm{~nm})$<smiles>CO[C@H]1O[C@H](C(=O)O)[C@H]2OC(C)(C)O[C@H]12</smiles>

$1 \mathrm{c}$<smiles>N#CC1=CCCCC1=O</smiles>

$4 \mathrm{~g}(2.0 \mathrm{eq})$<smiles>CO[C@H]1OC(C2O[C@@H](OC)[C@H]3OC(C)(C)O[C@H]23)C2OC(C)(C)O[C@@H]21</smiles>

3c: $0 \%$

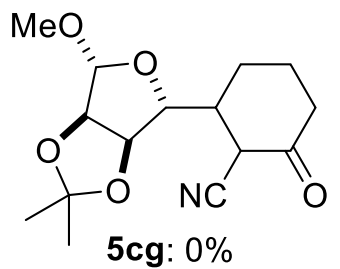

4g: $0 \%$

$\operatorname{Ir}\left[\mathrm{dF}\left(\mathrm{CF}_{3}\right) \mathrm{ppy}\right]_{2}$ (dtbbpy)PF 6 (2.5 mol\%) $\mathrm{K}_{2} \mathrm{HPO}_{4}(1.5 \mathrm{eq})$<smiles>CO[C@H]1O[C@H](C(=O)O)[C@H]2OC(C)(C)O[C@H]12</smiles>
blue LED $(448 \mathrm{~nm})$<smiles>CO[C@H]1O[C@@H](C2(C(C#N)C#N)CCCCC2)[C@@H]2OC(C)(C)O[C@H]12</smiles>

5ch: $0 \%$ 4h: $0 \%$

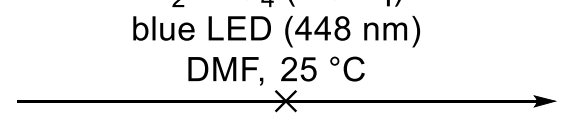

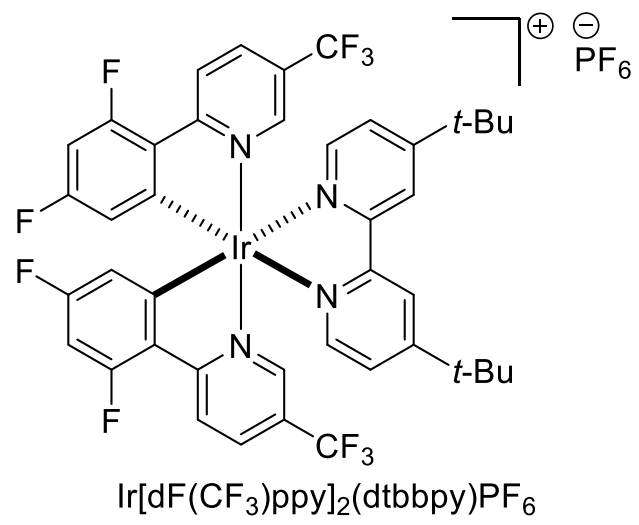




\section{References}

S1. Manley, D. W.; Walton, J. C. A Clean and Selective Radical Homocoupling Employing Carboxylic Acids with Titania Photoredox Catalysis. Org. Lett. 2014, 16, 5394-5397.

S2. Masuda, K.; Nagatomo, M.; Inoue, M. Direct Assembly of Multiply Oxygenated Carbon Chains by Decarbonylative Radical-Radical Coupling Reactions. Nat. Chem. 2017, 9, 207-212.

S3. Nagatomo, M.; Kamimura, D.; Matsui, Y.; Masuda, K.; Inoue, M. Et ${ }_{3}$ B-Mediated Twoand Three-Component Coupling Reactions via Radical Decarbonylation of $\alpha$ Alkoxyacyl Tellurides: Single-Step Construction of Densely Oxygenated Carboskeletons. Chem. Sci. 2015, 6, 2765-2769.

S4. Webb, E. W.; Park, J. B.; Cole, E. L.; Donnelly, D. J.; Bonacorsi, S. J.; Ewing, W. R.; Doyle, A. G. Nucleophilic (Radio)Fluorination of Redox-Active Esters via Radical-Polar Crossover Enabled by Photoredox Catalysis. J. Am. Chem. Soc. 2020, 142, 9493-9500.

S5. Kessinger, R.; Thilgen, C.; Mordasini, T.; Diederich, F. Optically Active Macrocyclic cis-3 Bis-Adducts of C60:Regio- and Stereoselective Synthesis, Exciton Chirality Coupling, and Determination of the Absolute Configuration, and First Observation of Exciton Coupling between Fullerene Chromophores in a Chiral Environment. Helv. Chim. Acta 2000, 83, 3069-3096.

S6. Guarna, A.; Guidi, A.; Machetti, F.; Menchi, G.; Occhiato, E. G.; Scarpi, D.; Sisi, S.; Trabocchi, A. Synthesis and Reactivity of Bicycles Derived from Tartaric Acid and $\alpha-$ Amino Acids: A Novel Class of Conformationally Constrained Dipeptide Isosteres Based upon Enantiopure 3-Aza-6,8-dioxabicyclo[3.2.1] octane-7-carboxylic Acid. J. Org. Chem. 1999, 64, 7347-7364.

S7. Kuwana, D.; Nagatomo, M.; Inoue, M. Total Synthesis of 5-epi-Eudesm-4(15)-ene$1 \beta, 6 \beta$-diol via Decarbonylative Radical Coupling Reaction. Org. Lett. 2019, 21, 7619-7623.

S8. Fleming, F. F.; Huang, A.; Sharief, V. A.; Pu, Y. Unsaturated Nitriles: Precursors for a Domino Ozonolysis-Aldol Synthesis of Oxonitriles. J. Org. Chem. 1999, 64, 2830-2834.

S9. Urabe, D.; Yamaguchi, H.; Inoue, M. Application of $\alpha$-Alkoxy Bridgehead Radical for Coupling of Oxygenated Carbocycles. Org. Lett. 2011, 13, 4778-4781.

S10. Góra, M.; Kozik, B.; Jamroży, K.; Łuczyński, M. K.; Brzuzan, P.; Woźny, M. SolventFree Condensations of Ketones with Malononitrile Catalysed by Methanesulfonic Acid/Morpholine System. Green Chem. 2009, 11, 863-867.

S11. Chu, L.; Ohta, C.; Zuo, Z.; MacMillan, D. W. C. Carboxylic Acids as A Traceless Activation Group for Conjugate Additions: A Three-Step Synthesis of ( \pm )-Pregabalin. $J$. Am. Chem. Soc. 2014, 136, 10886-10889. 


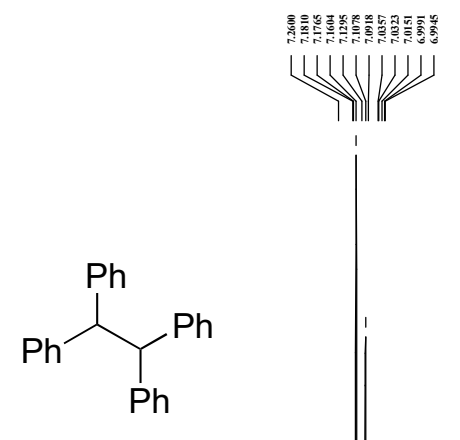

$3 a$

${ }^{1} \mathrm{H}$ NMR $\left(400 \mathrm{MHz}, \mathrm{CDCl}_{3}\right)$

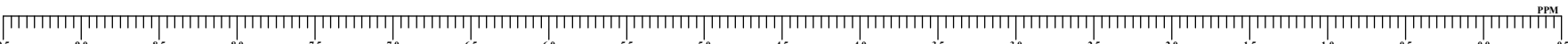

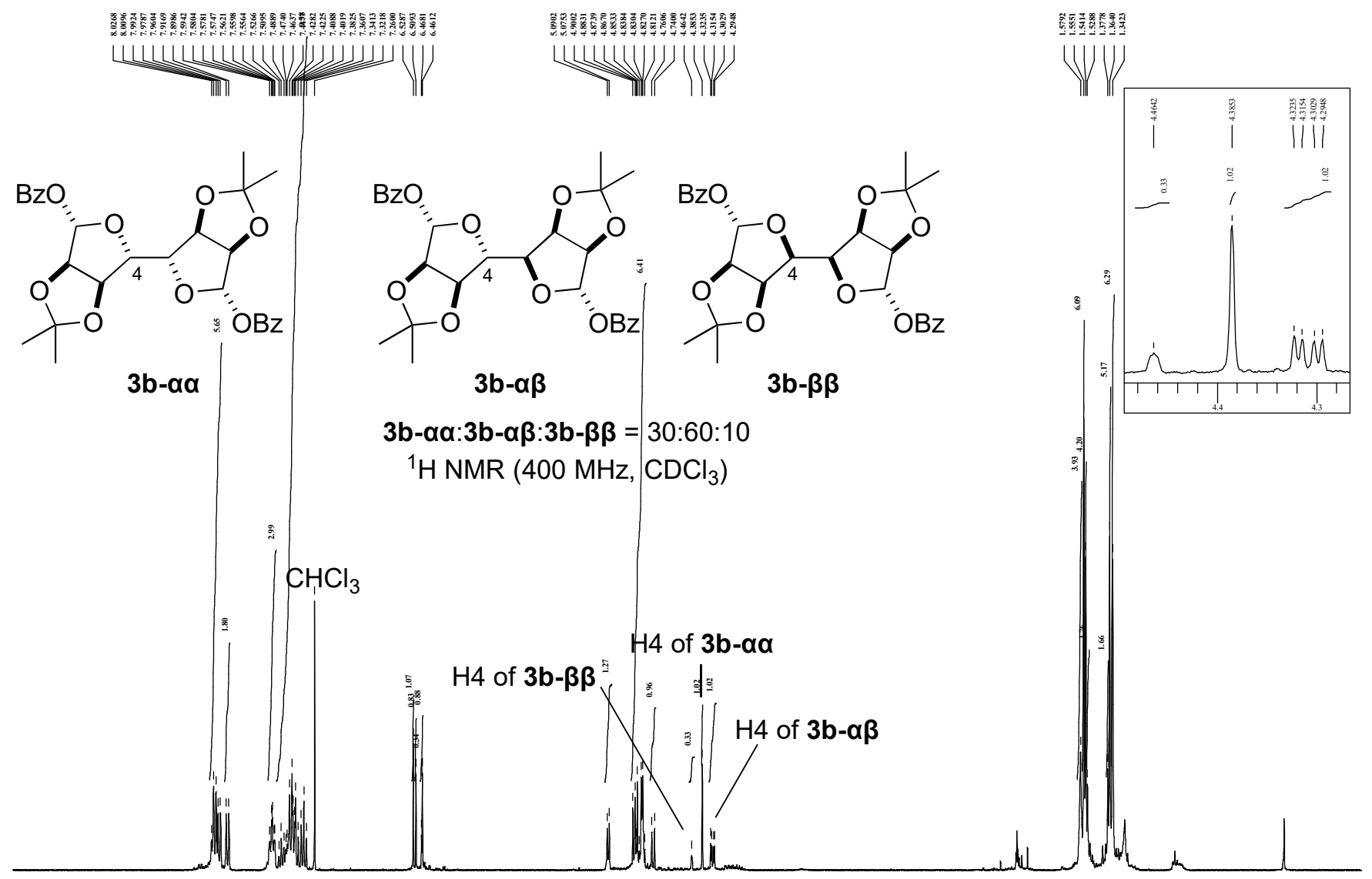

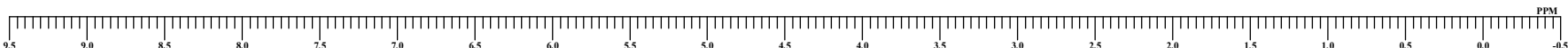




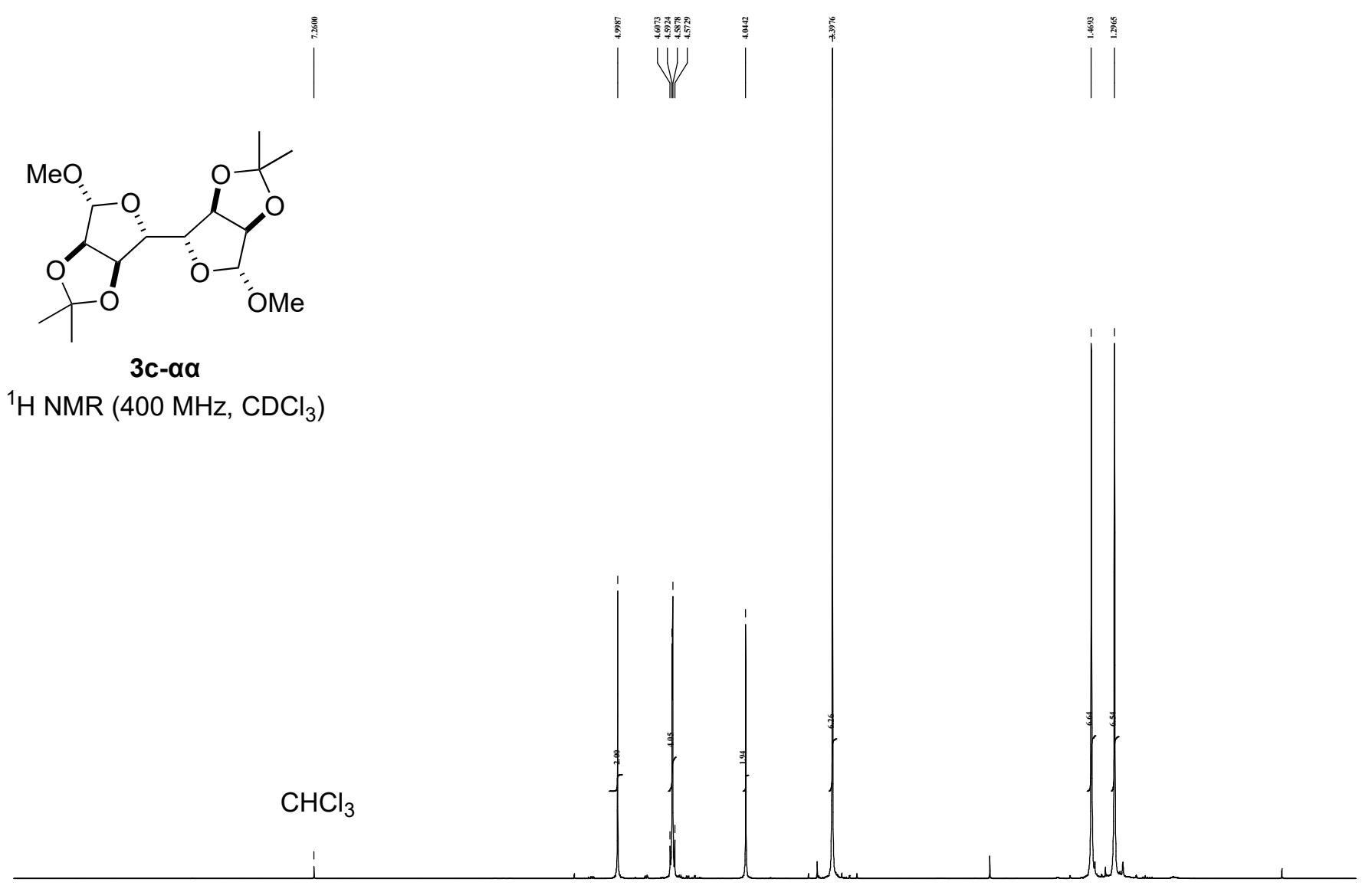

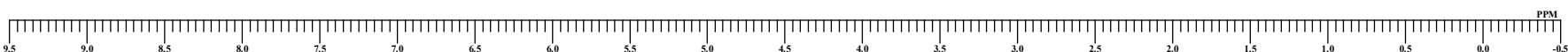

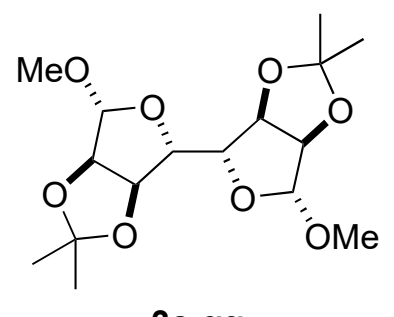

3c- $\alpha \alpha$

${ }^{13} \mathrm{C}\left\{{ }^{1} \mathrm{H}\right\} \operatorname{NMR}\left(100 \mathrm{MHz}, \mathrm{CDCl}_{3}\right)$

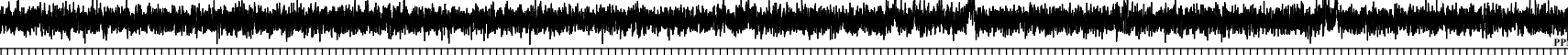

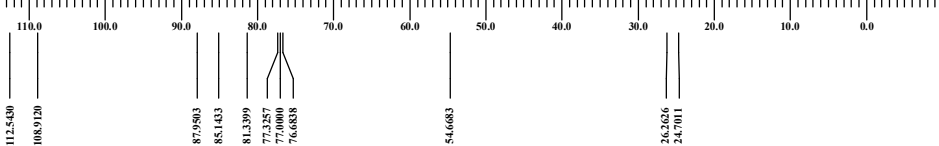




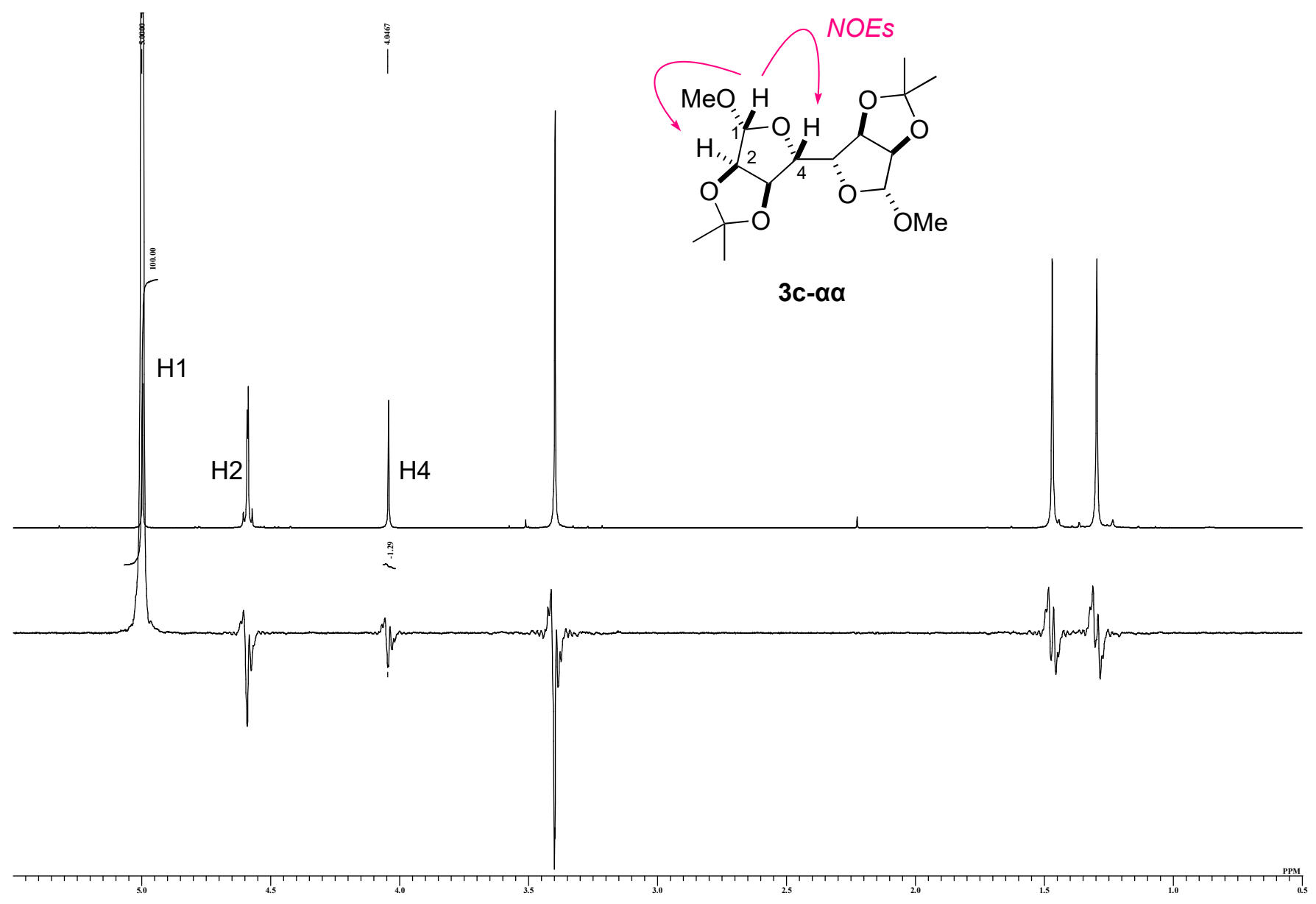



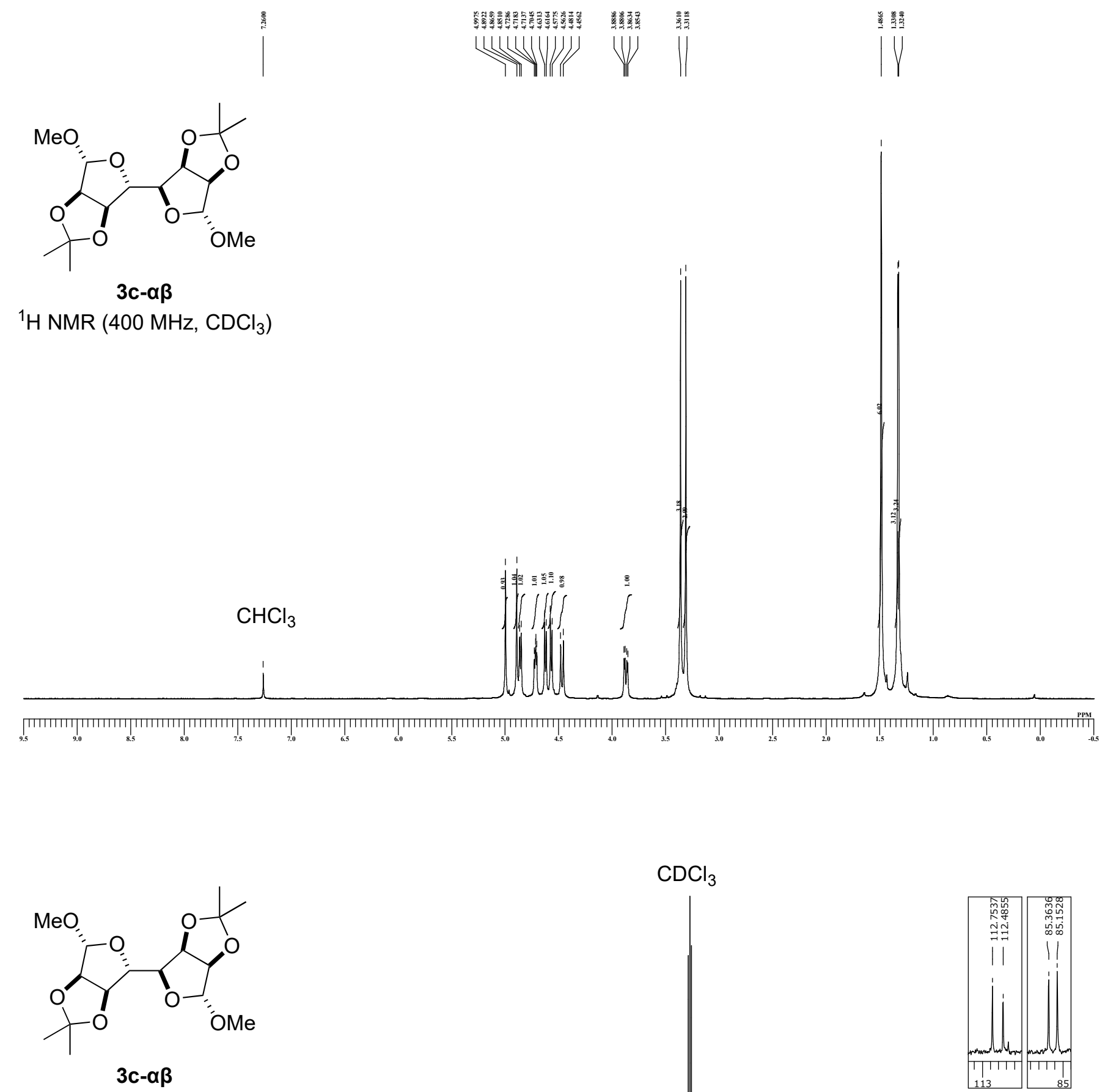

${ }^{13} \mathrm{C}\left\{{ }^{1} \mathrm{H}\right\} \mathrm{NMR}\left(100 \mathrm{MHz}, \mathrm{CDCl}_{3}\right)$

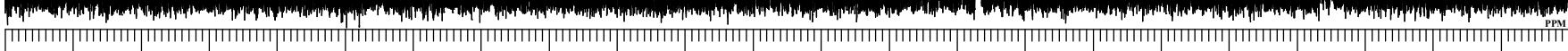

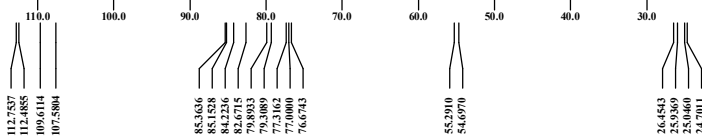


<smiles>CC1(C)OC2C(O1)[C@H](Cc1ccccc1)OC2[C@H]1OC(Cc2ccccc2)[C@H]2OC(C)(C)OC21</smiles>

$3 d-\alpha a$

${ }^{1} \mathrm{H}$ NMR $\left(400 \mathrm{MHz}, \mathrm{CDCl}_{3}\right)$

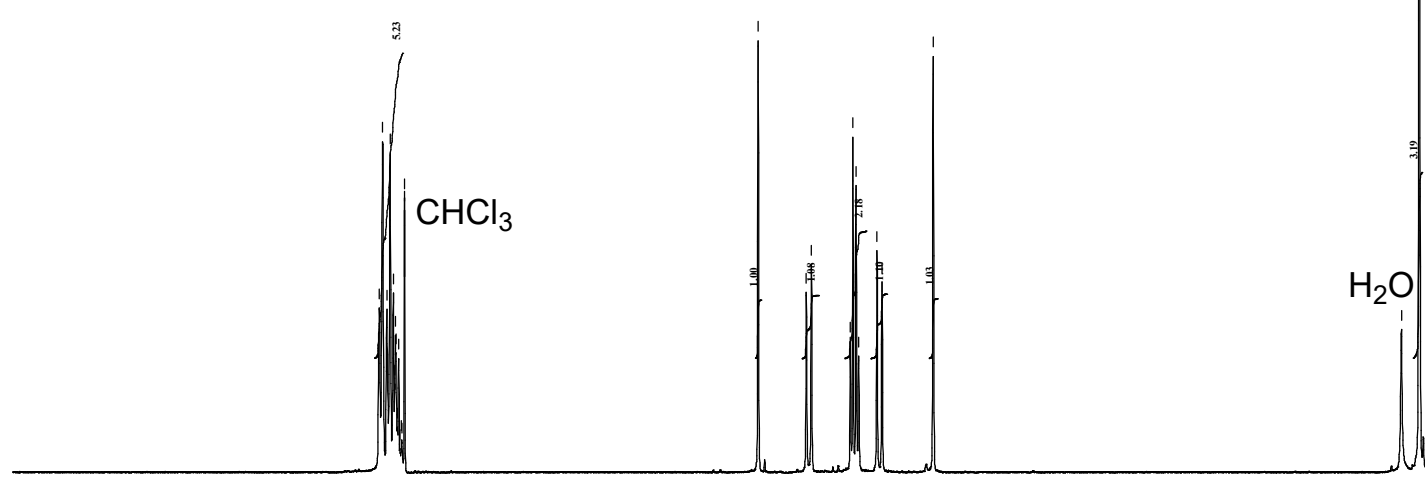

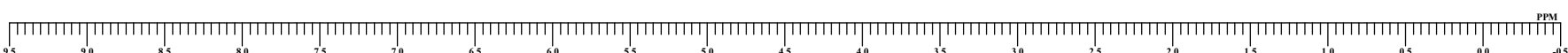<smiles>CC1(C)OC(O)C(C2OC(OCc3ccccc3)C3OC(C)(C)OC32)O1</smiles>

$3 d-\alpha \alpha$

${ }^{13} \mathrm{C}\left\{{ }^{1} \mathrm{H}\right\} \mathrm{NMR}\left(100 \mathrm{MHz}, \mathrm{CDCl}_{3}\right)$

$\mathrm{CDCl}_{3}$

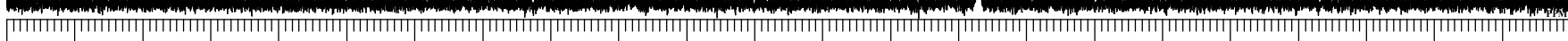

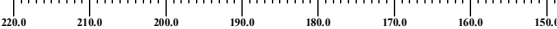

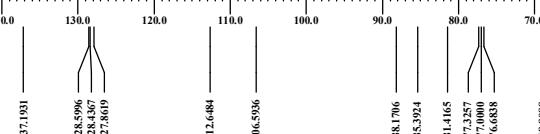




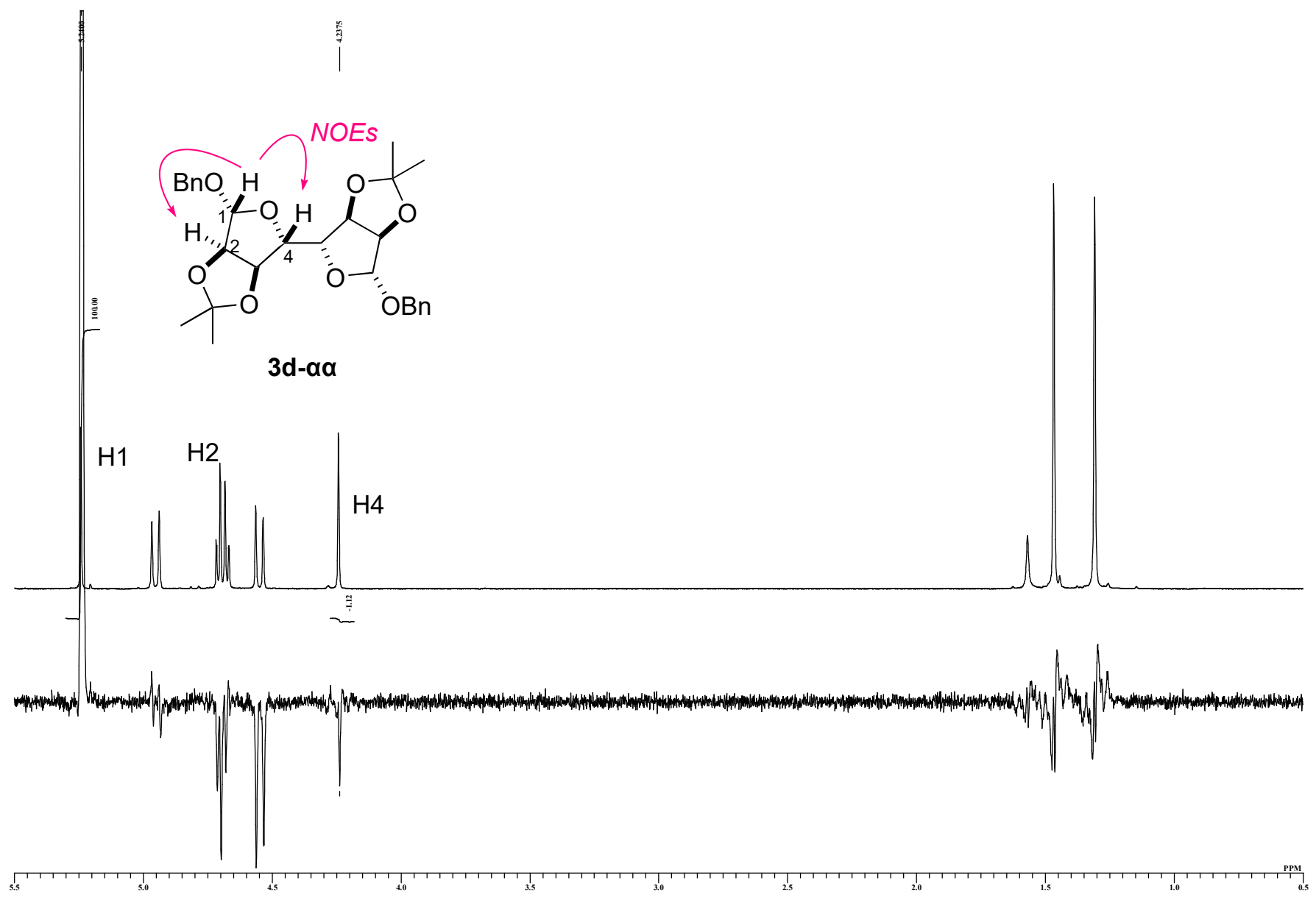




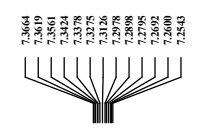

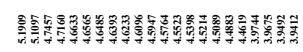

แMWWWHDW

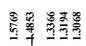

IIV<smiles>CC1(C)OC2C(Cc3ccccc3)OC(C3OC(O[Ga])C4OC(C)(C)OC43)C2O1</smiles>

$3 d-\alpha \beta$

${ }^{1} \mathrm{H}$ NMR $\left(400 \mathrm{MHz}, \mathrm{CDCl}_{3}\right)$
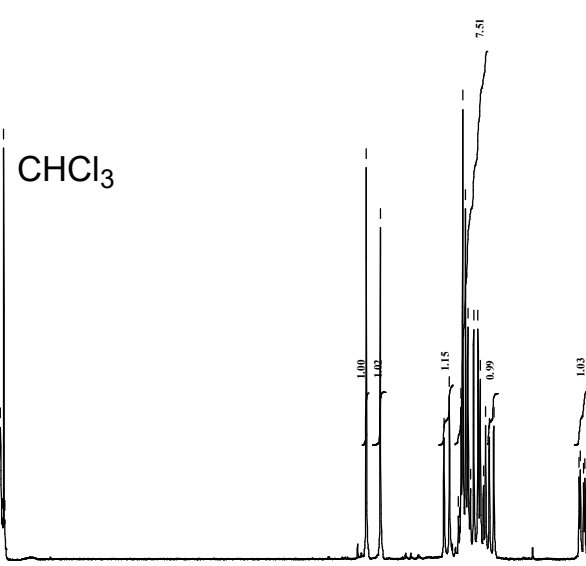

$\mathrm{H}_{2} \mathrm{O}$

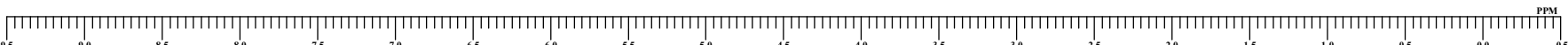

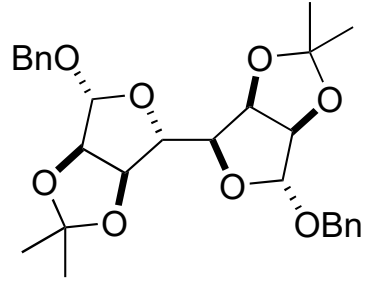

3d- $\alpha \beta$

${ }^{13} \mathrm{C}\left\{{ }^{1} \mathrm{H}\right\}$ NMR $\left(100 \mathrm{MHz}, \mathrm{CDCl}_{3}\right)$
$\mathrm{CDCl}_{3}$

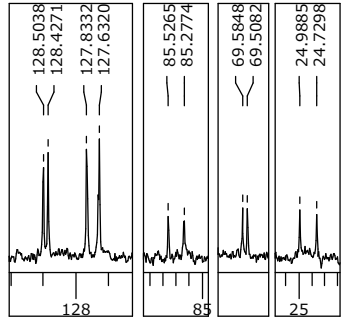




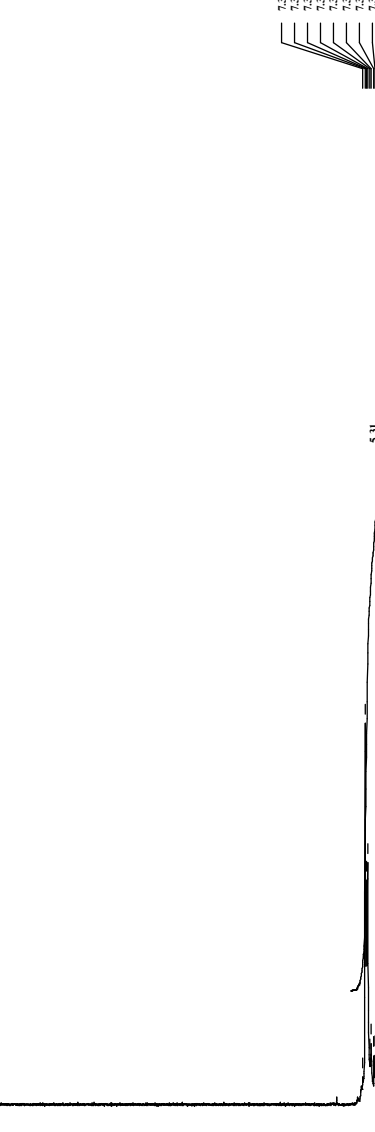<smiles>CC1(C)OC2C(c3ccccc3)O[C@@H](O)C3OC(C)(C)OC3C2O1</smiles>

\section{3d- $\beta \beta$}

${ }^{1} \mathrm{H}$ NMR $\left(400 \mathrm{MHz}, \mathrm{CDCl}_{3}\right)$

$\mathrm{CHCl}_{3}$

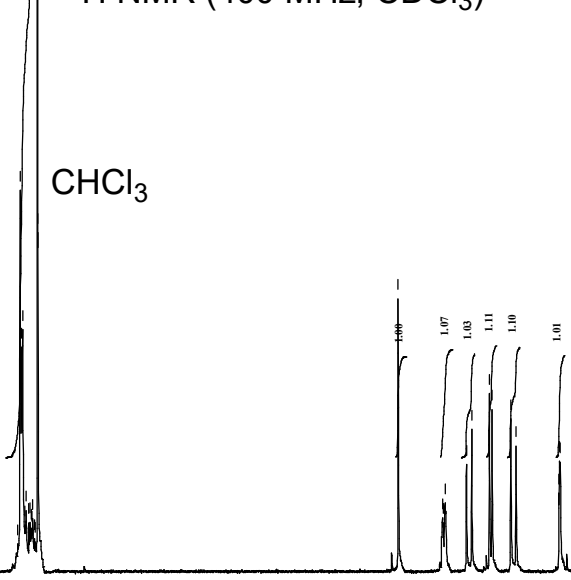

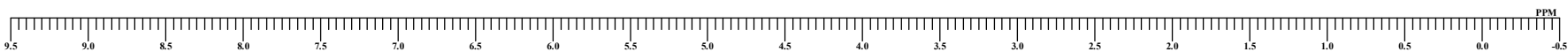

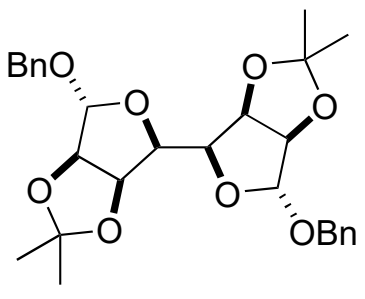

$3 d-\beta \beta$

${ }^{13} \mathrm{C}\left\{{ }^{1} \mathrm{H}\right\}$ NMR $\left(100 \mathrm{MHz}, \mathrm{CDCl}_{3}\right)$
$\mathrm{CDCl}_{3}$

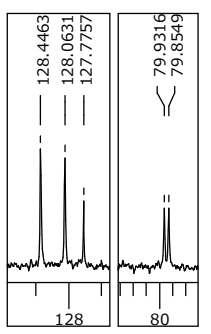

C.

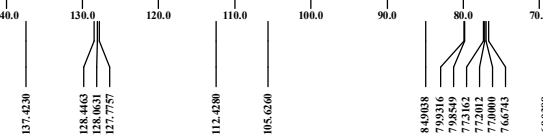




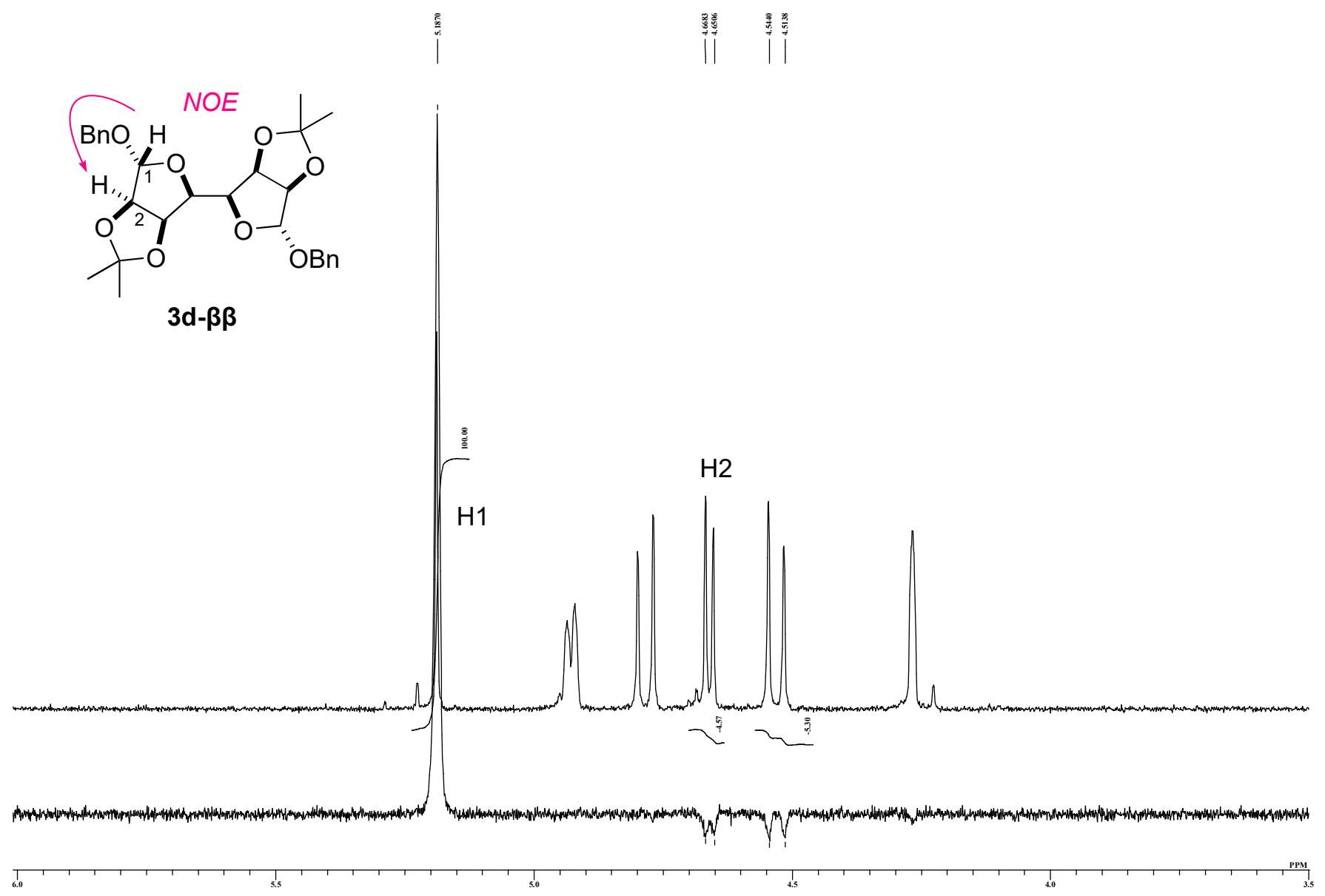




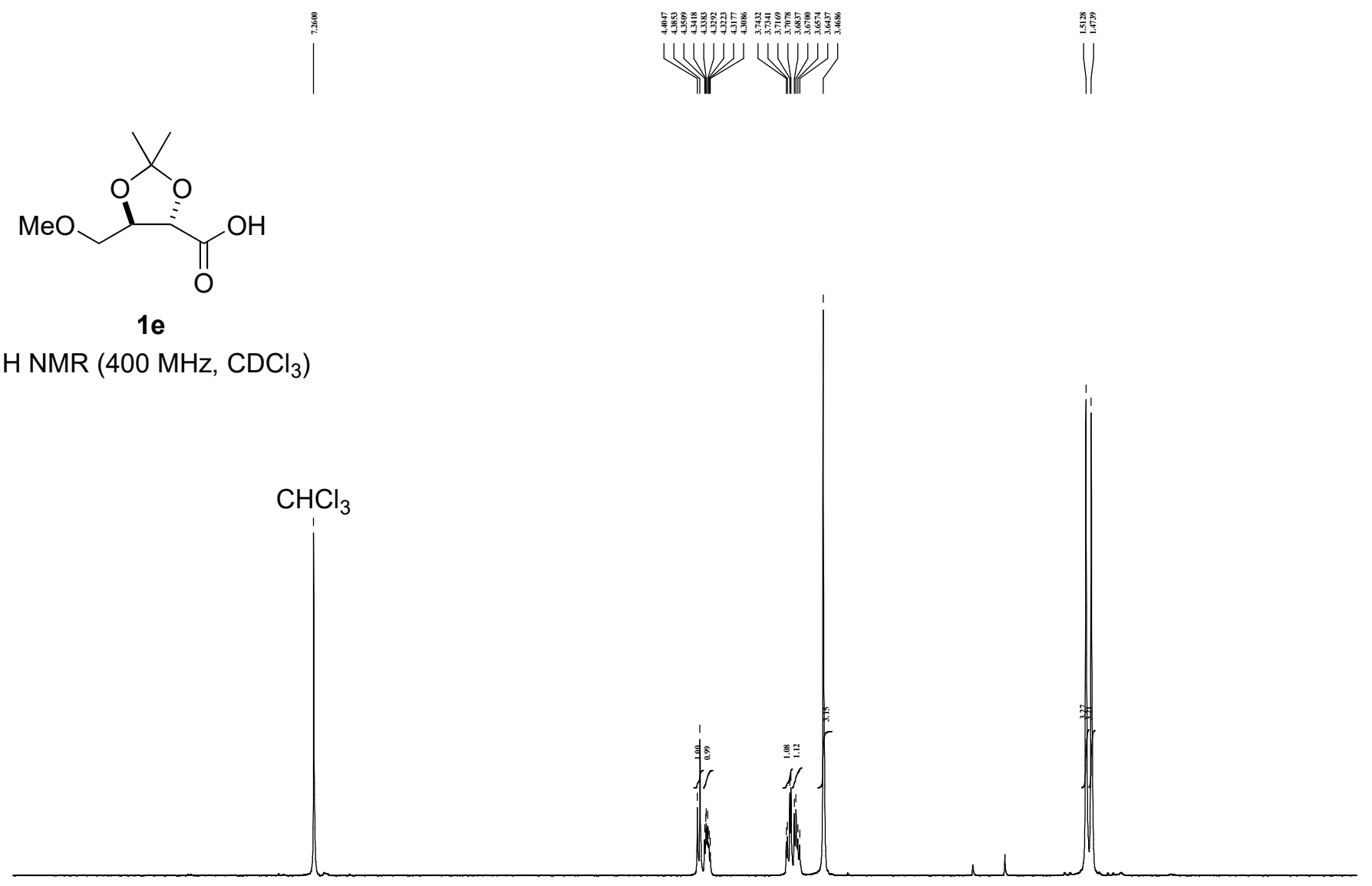

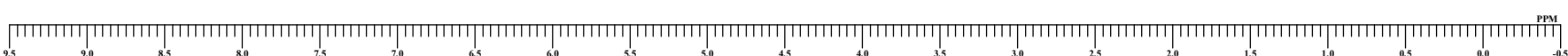<smiles>COCC1OC(C)(C)OC1C(=O)O</smiles>

$1 e$

${ }^{13} \mathrm{C}\left\{{ }^{1} \mathrm{H}\right\} \mathrm{NMR}\left(100 \mathrm{MHz}, \mathrm{CDCl}_{3}\right)$

$\mathrm{CDCl}_{3}$

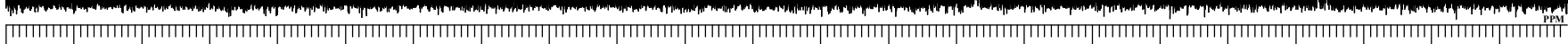

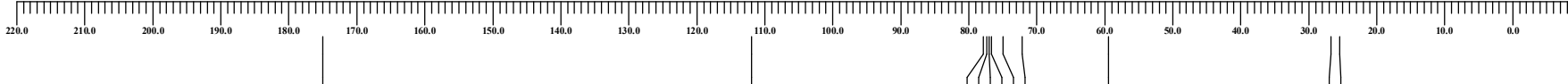

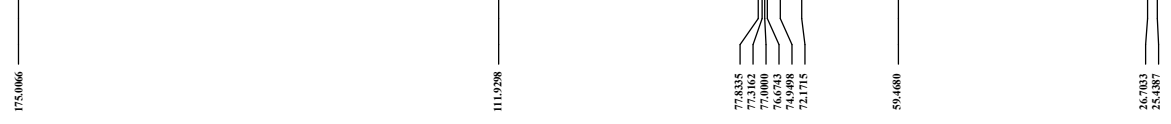




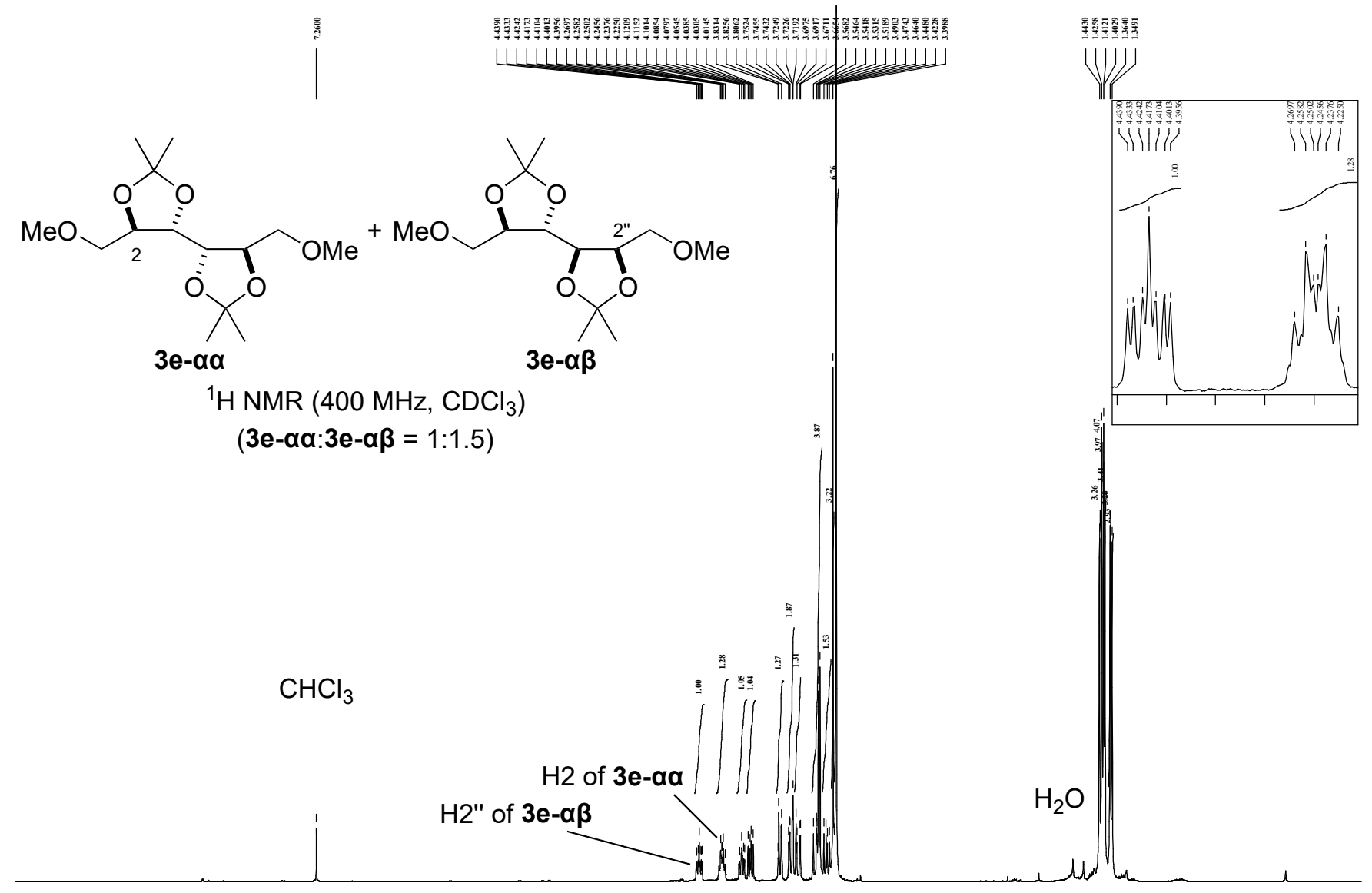

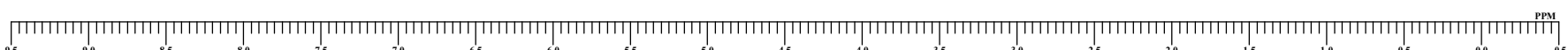

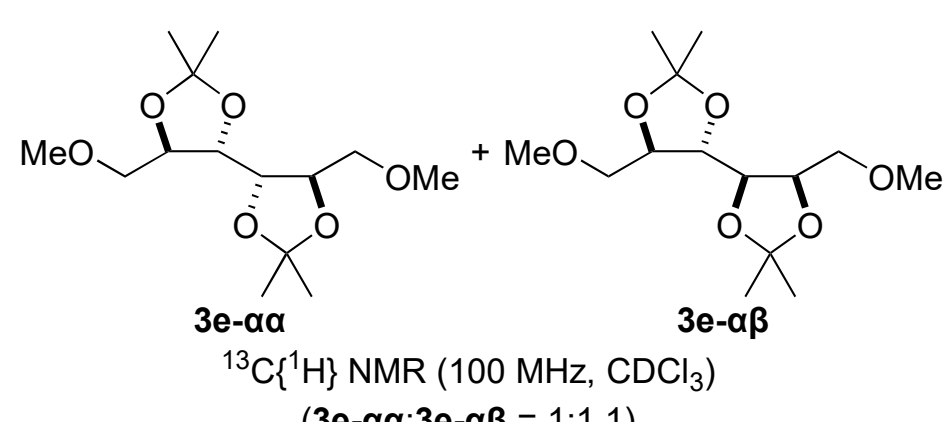

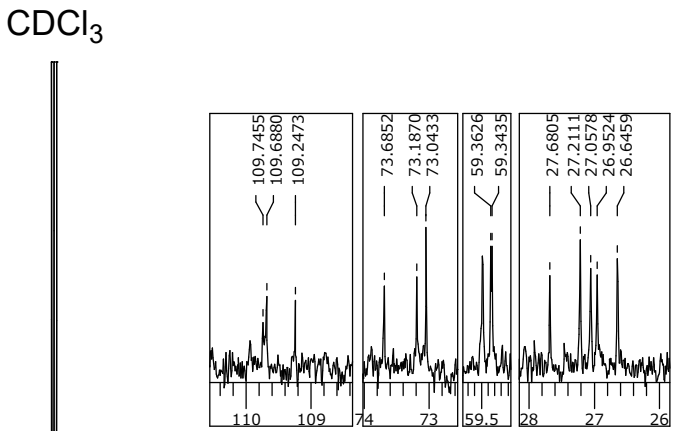

$(3 e-\alpha \alpha: 3 e-\alpha \beta=1: 1.1)$

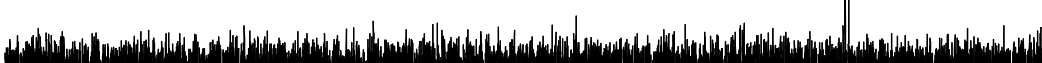

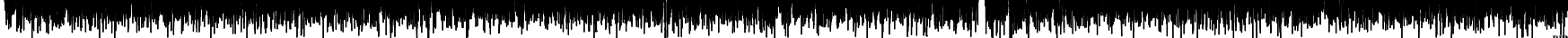

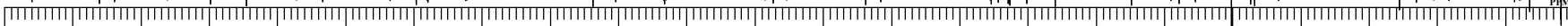

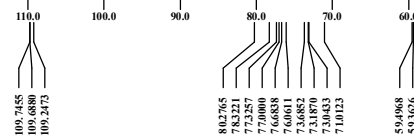




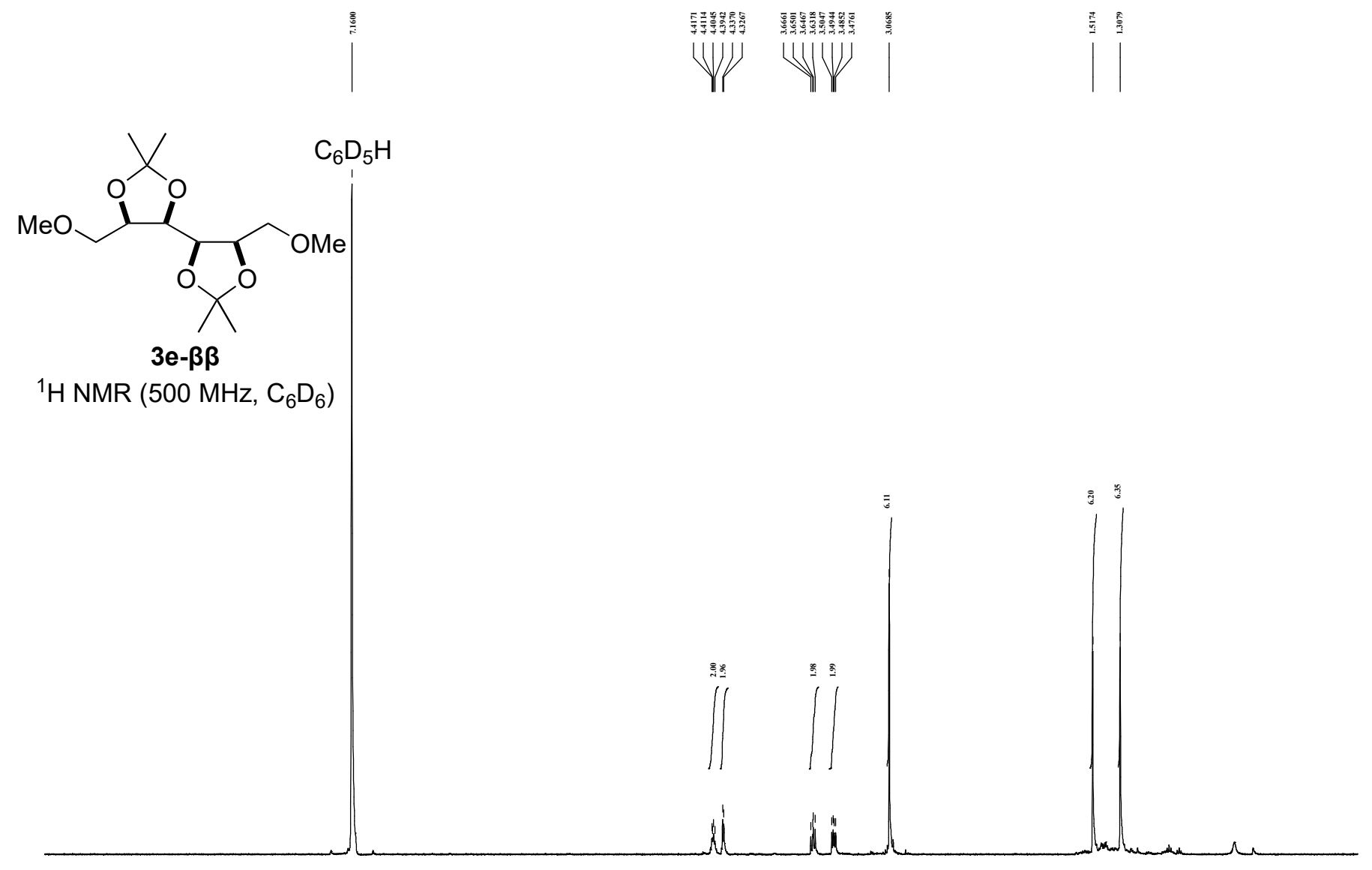

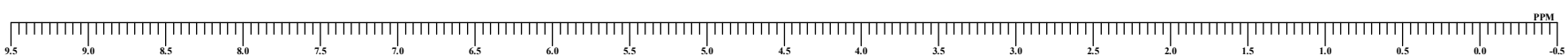

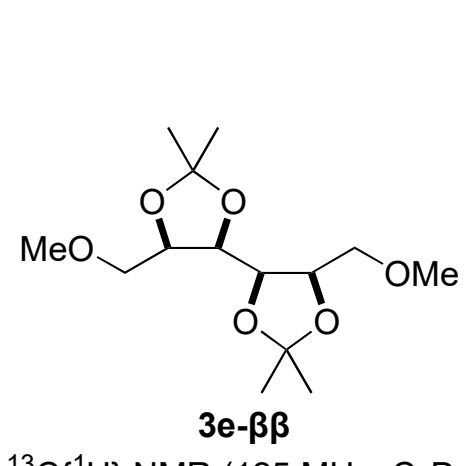

$\mathrm{C}_{6} \mathrm{D}_{6}$

${ }^{13} \mathrm{C}\left\{{ }^{1} \mathrm{H}\right\}$ NMR $\left(125 \mathrm{MHz}, \mathrm{C}_{6} \mathrm{D}_{6}\right)$

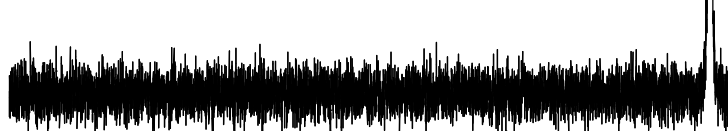

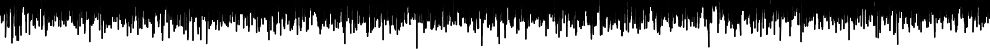
(

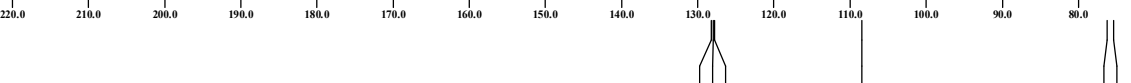




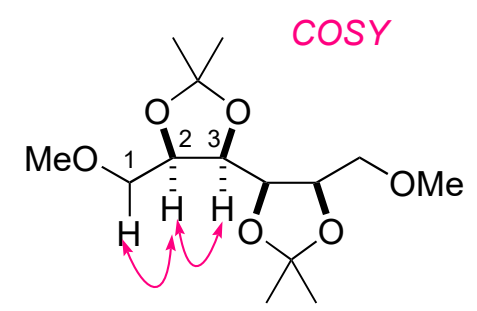

$3 e-\beta \beta$

$\operatorname{cosy}\left(500 \mathrm{MHz}, \mathrm{C}_{6} \mathrm{D}_{6}\right)$

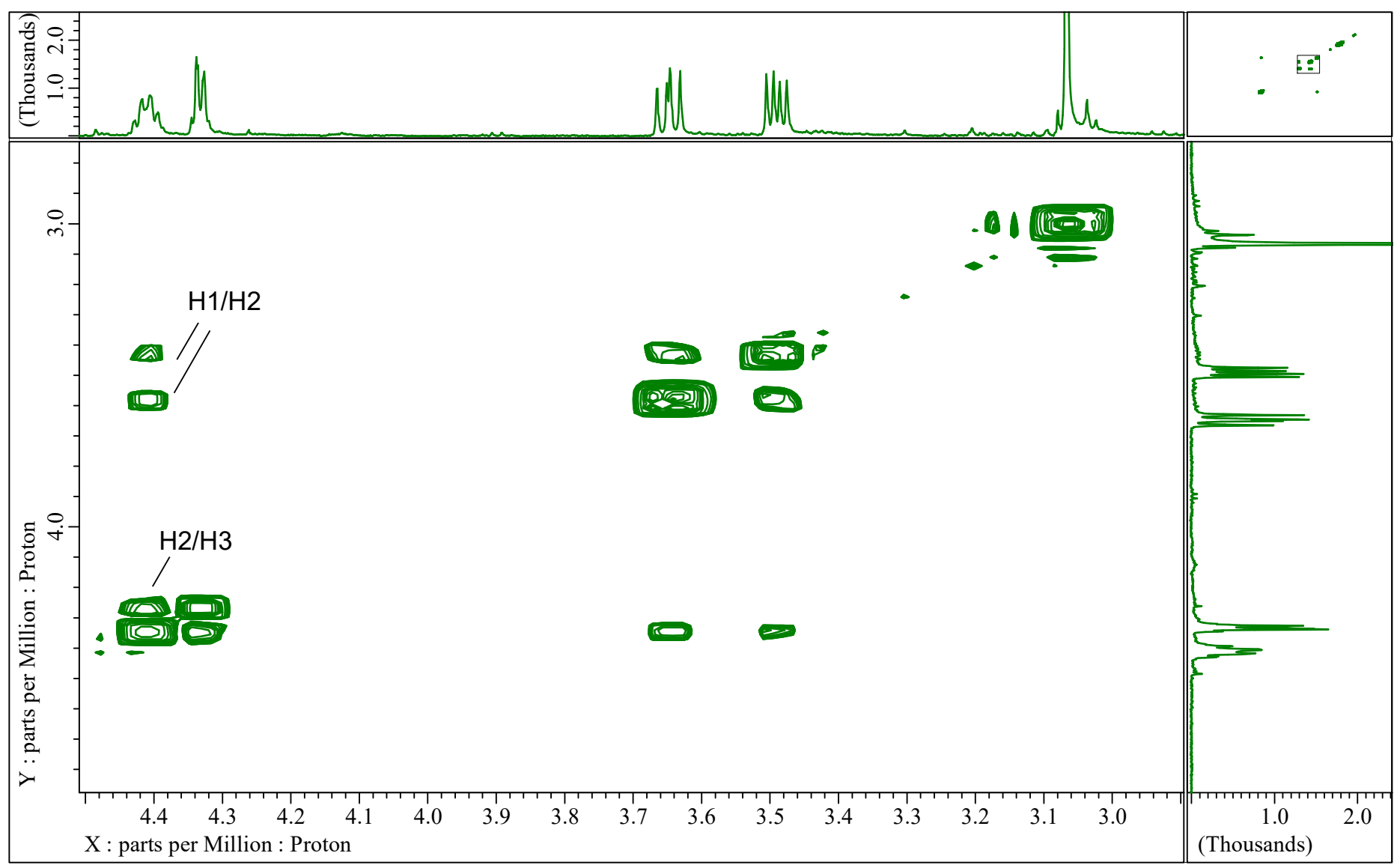




$$
\mathrm{H}^{\prime \prime}
$$


<smiles>COC(=O)C1OC(C)(C)O[C@H]1[C@H]1OC(C)(C)OC1C(=O)OC</smiles>

$3 f-\alpha \alpha$

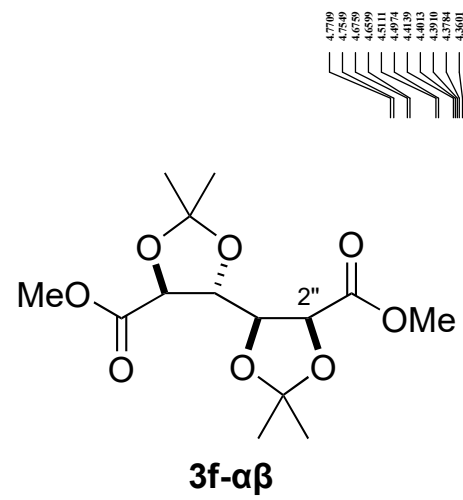

3f- $\alpha \boldsymbol{\beta}$

3f- $\mathbf{\alpha} \mathbf{\alpha}: \mathbf{3 f}-\boldsymbol{\alpha} \boldsymbol{\beta}=\mathbf{3}=\mathbf{5 5}$

${ }^{1} \mathrm{H}$ NMR $\left(400 \mathrm{MHz}, \mathrm{CDCl}_{3}\right)$

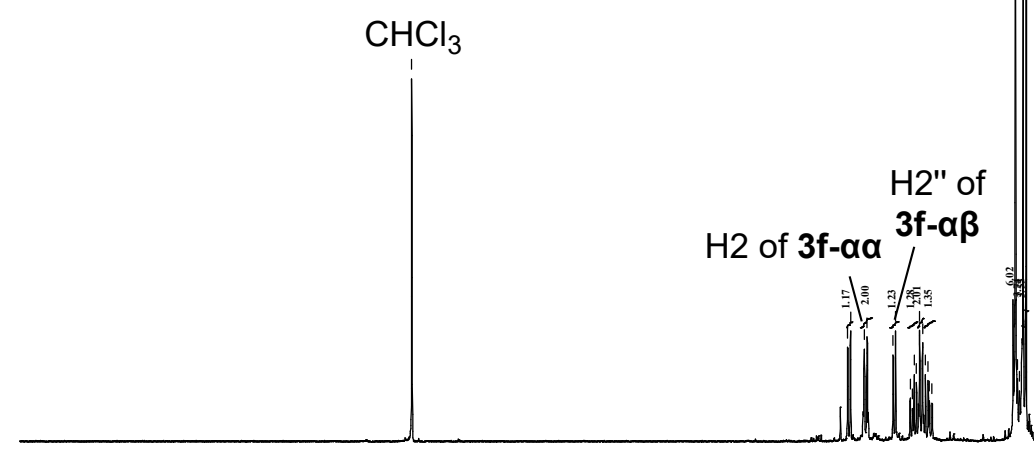

\section{年}

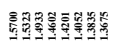

WW

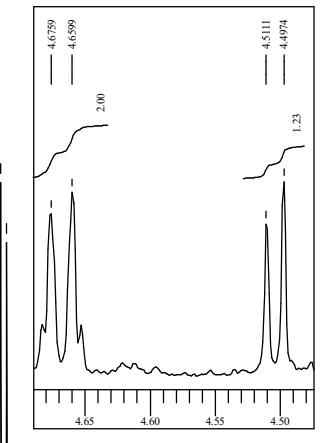

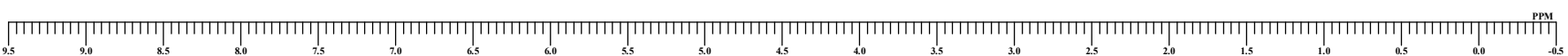
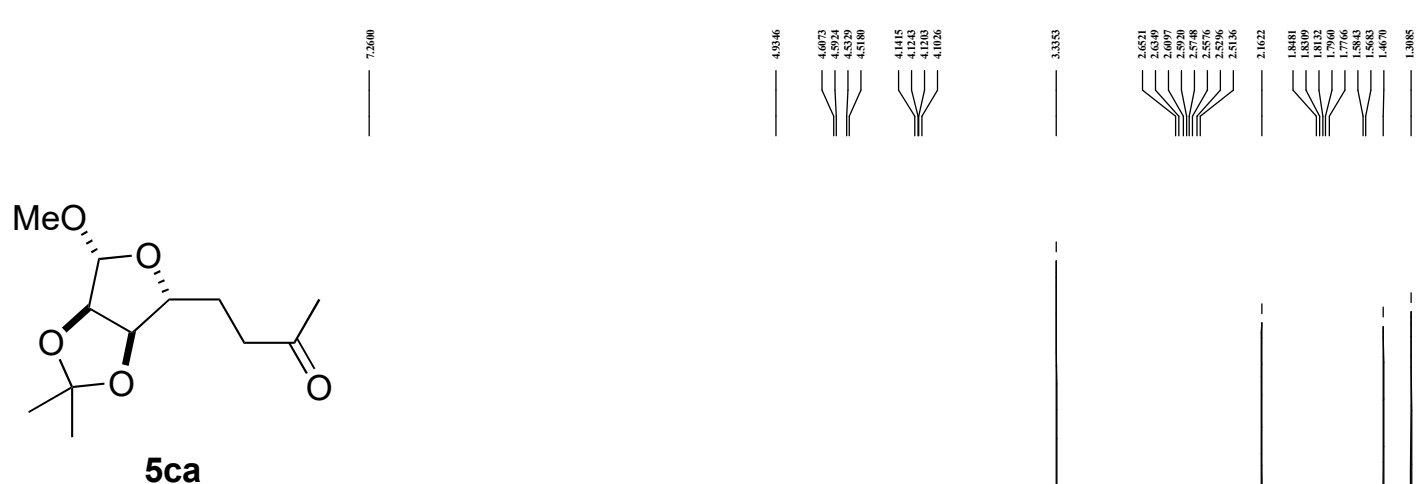

${ }^{1} \mathrm{H}$ NMR $\left(400 \mathrm{MHz}, \mathrm{CDCl}_{3}\right)$

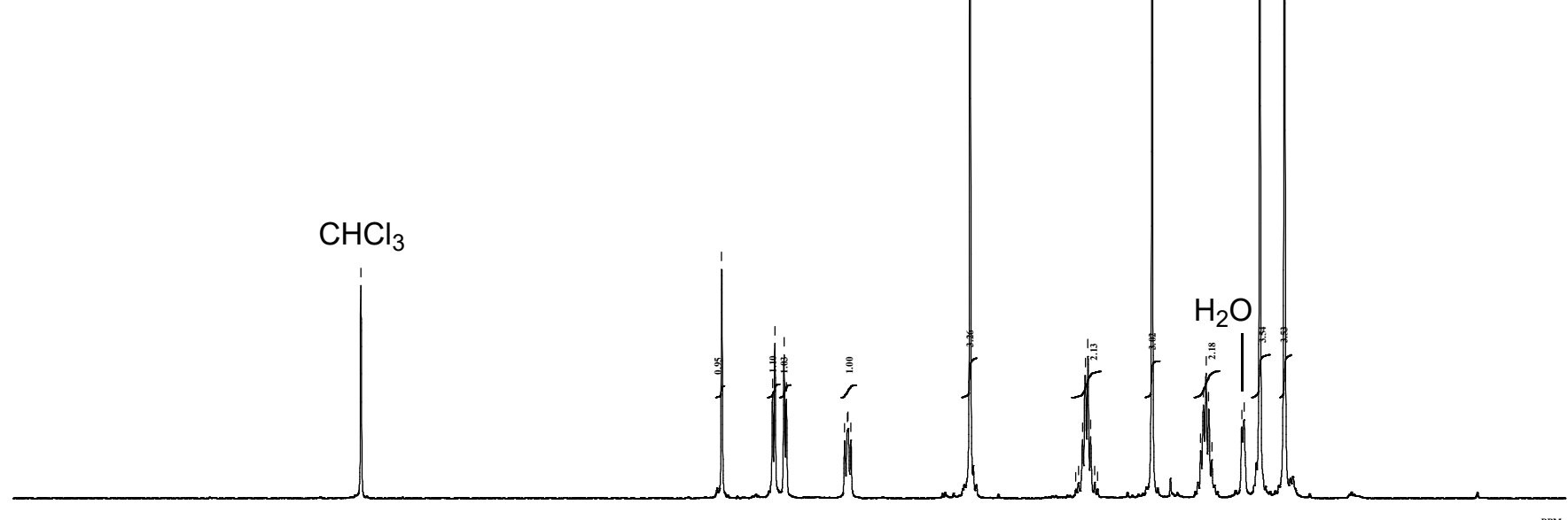




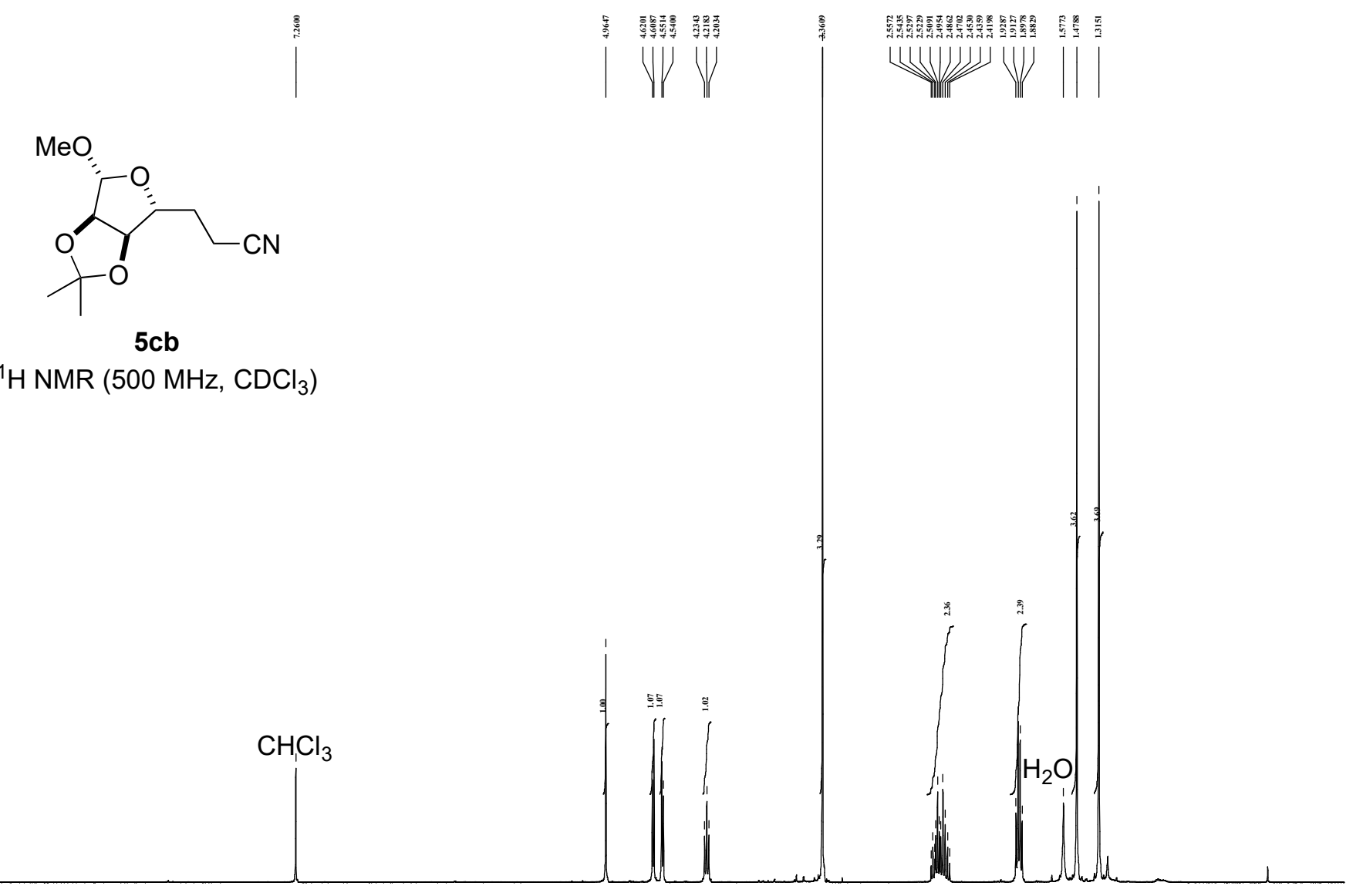

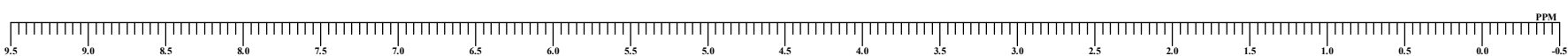

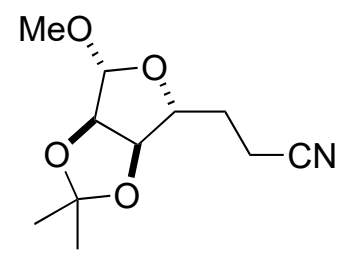

$5 \mathrm{cb}$

${ }^{13} \mathrm{C}\left\{{ }^{1} \mathrm{H}\right\} \operatorname{NMR}\left(125 \mathrm{MHz}, \mathrm{CDCl}_{3}\right)$

$\mathrm{CDCl}_{3}$

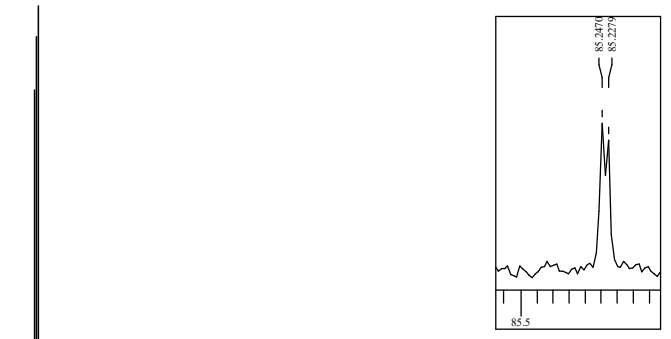

(1) $\underset{220.0}{210.0} \underset{200.0}{20.0}$

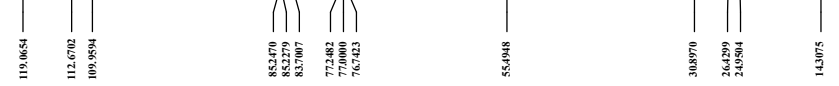




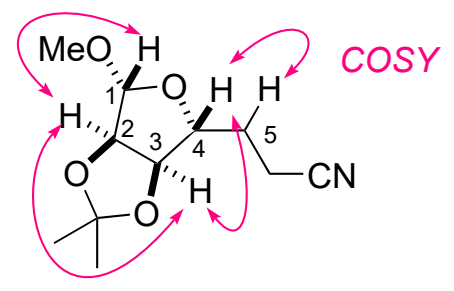

$5 \mathrm{cb}$

$\operatorname{cosY}\left(500 \mathrm{MHz}, \mathrm{CDCl}_{3}\right)$

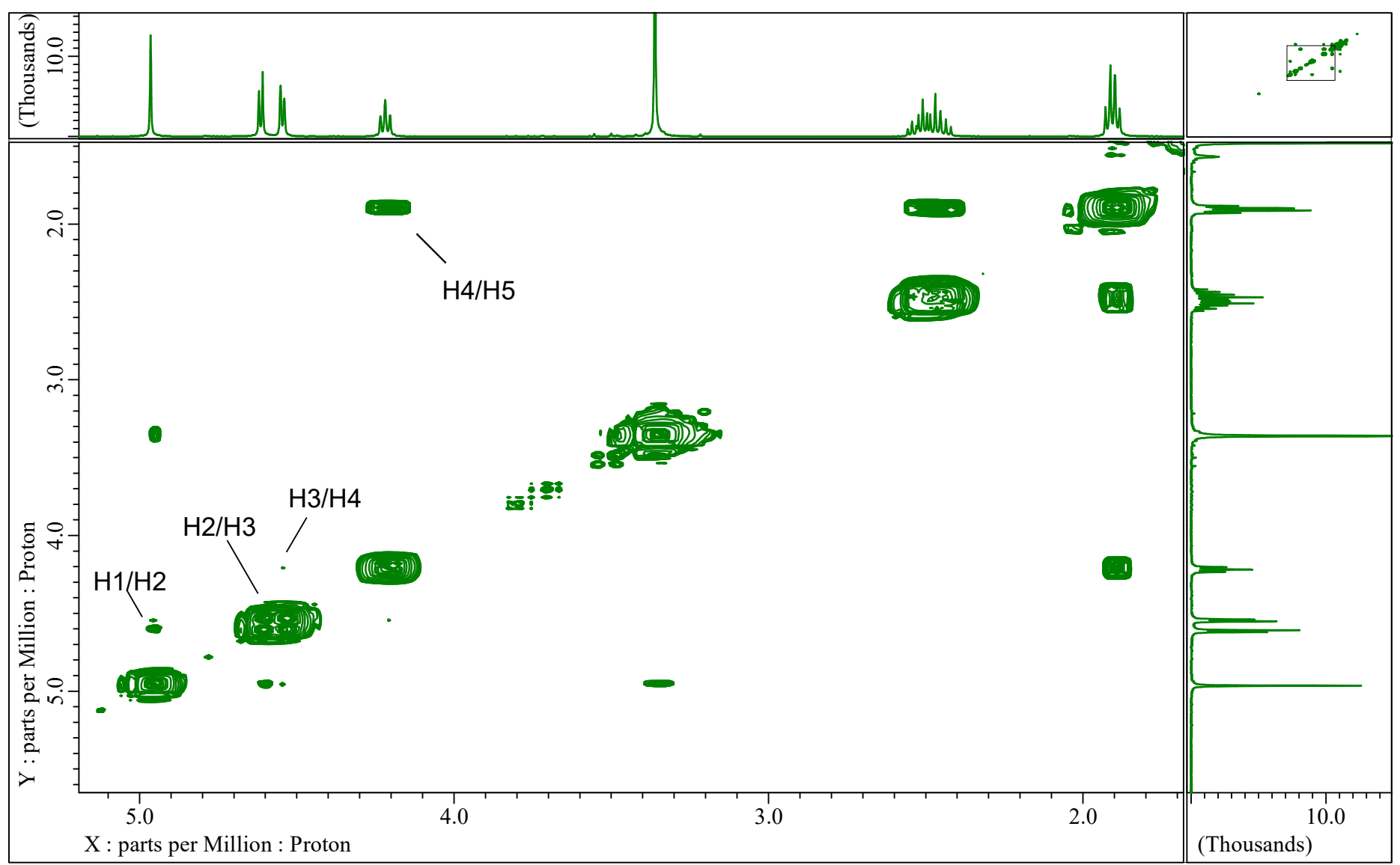




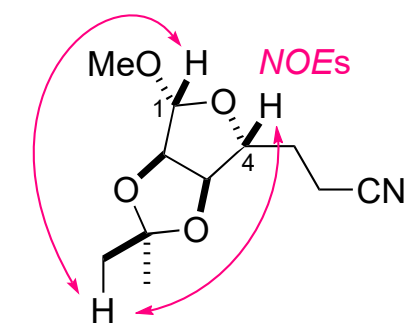

$5 \mathrm{cb}$

NOESY $\left(500 \mathrm{MHz}, \mathrm{CDCl}_{3}\right)$

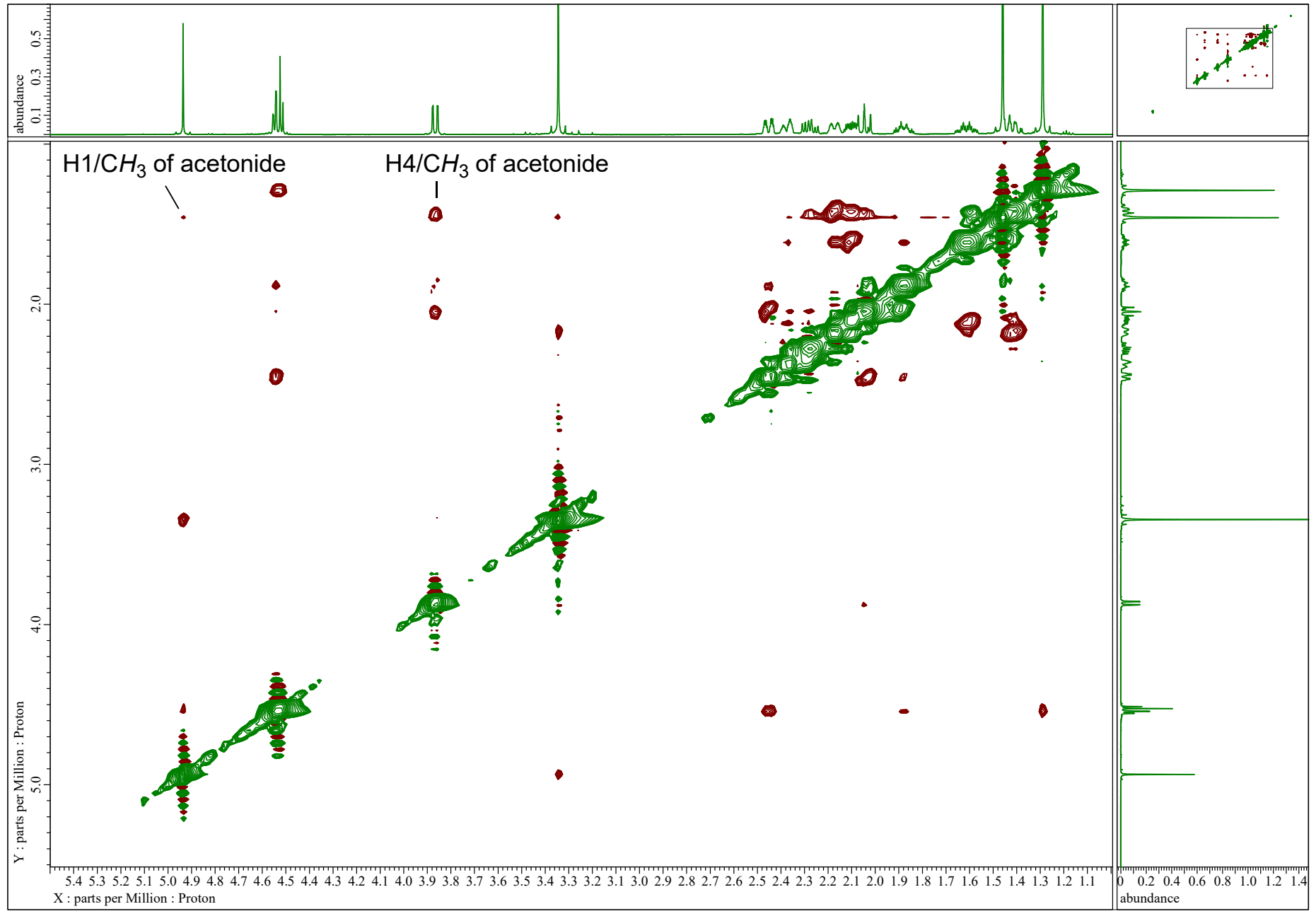



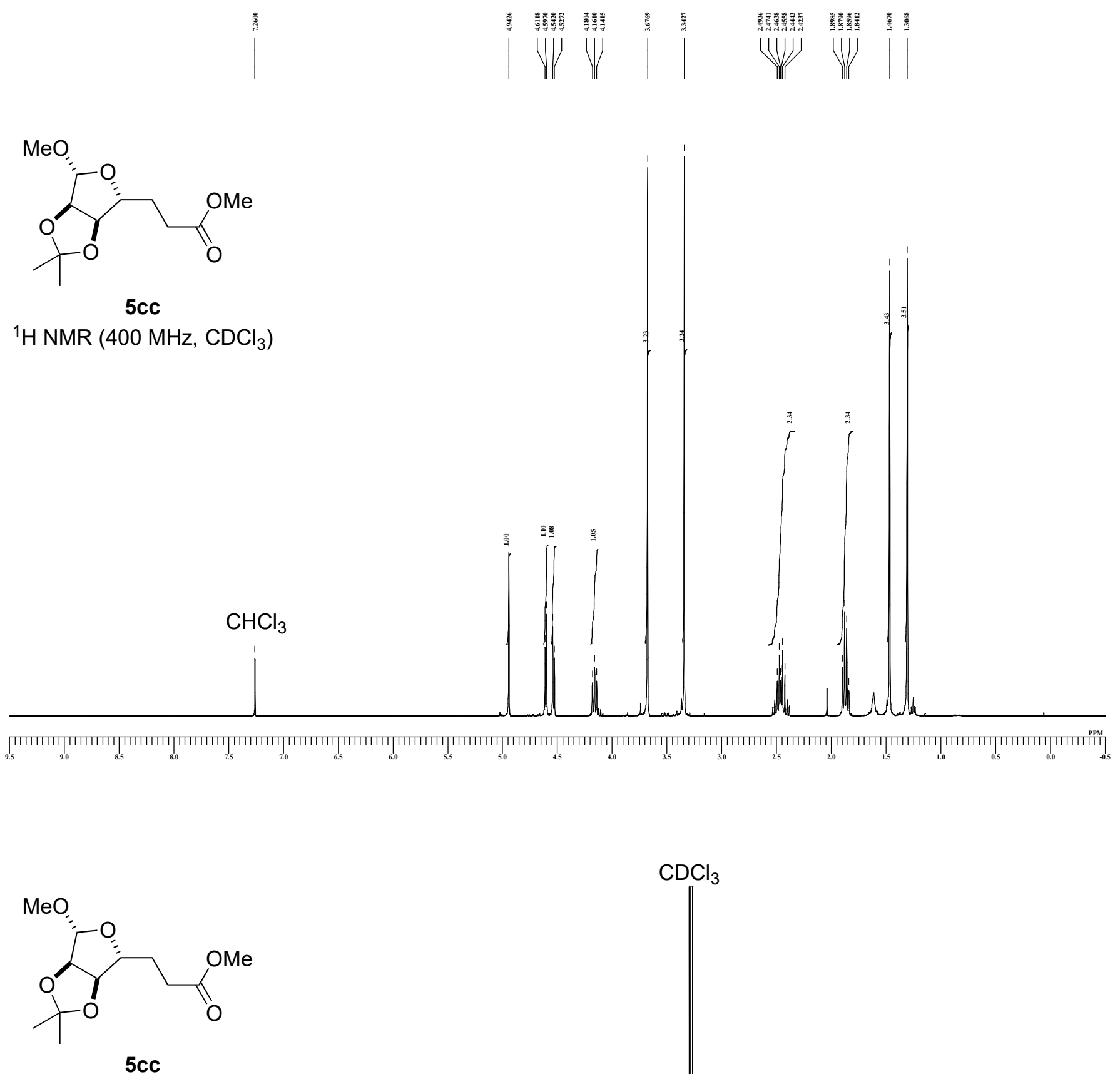

${ }^{13} \mathrm{C}\left\{{ }^{1} \mathrm{H}\right\}$ NMR $\left(100 \mathrm{MHz}, \mathrm{CDCl}_{3}\right)$
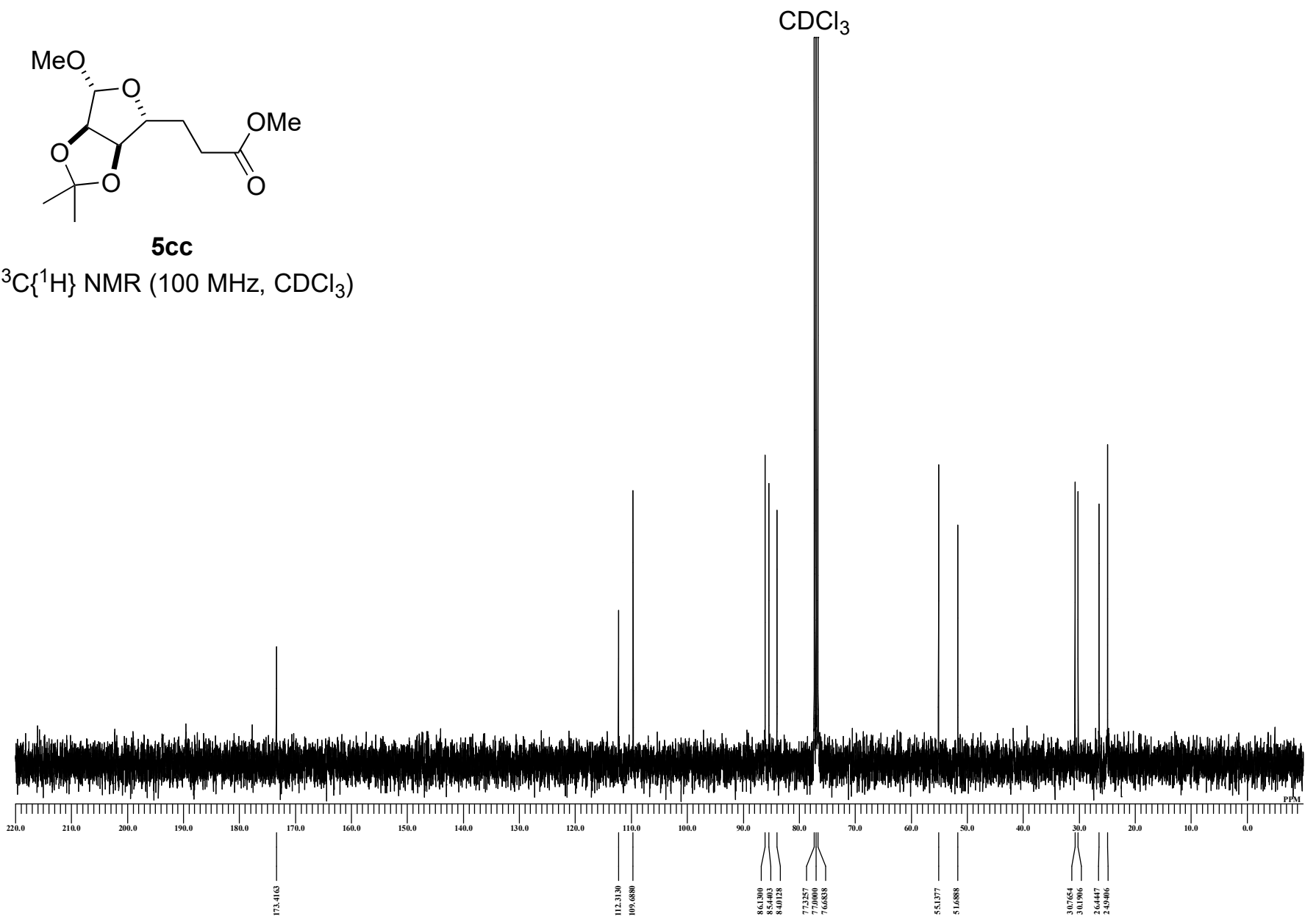


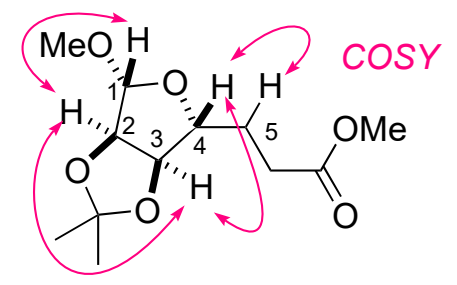

$5 \mathrm{cc}$

$\operatorname{COSY}\left(500 \mathrm{MHz}, \mathrm{CDCl}_{3}\right)$

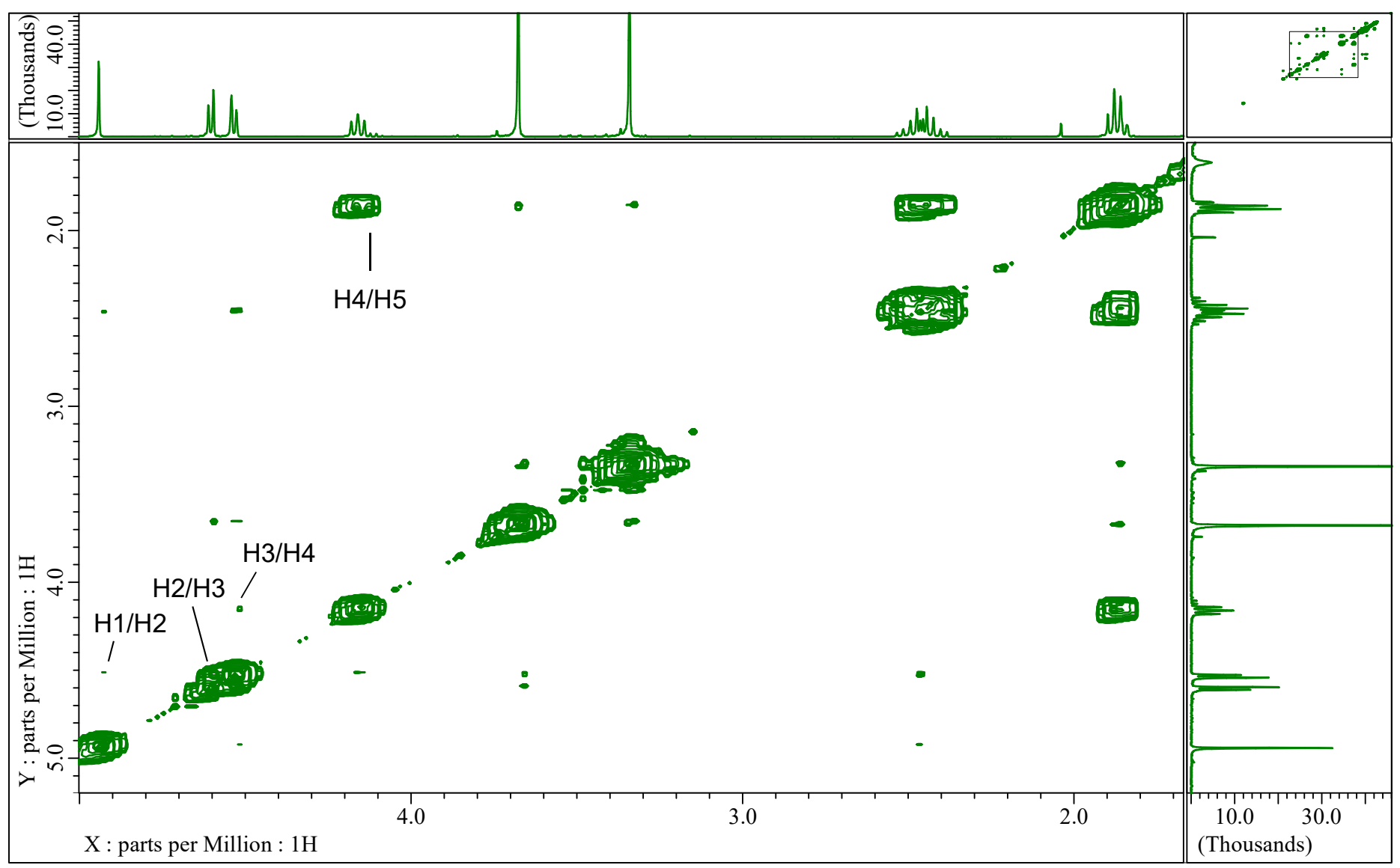




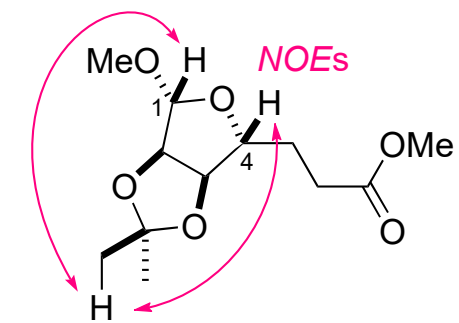

$5 \mathrm{cc}$

NOESY $\left(500 \mathrm{MHz}, \mathrm{CDCl}_{3}\right)$

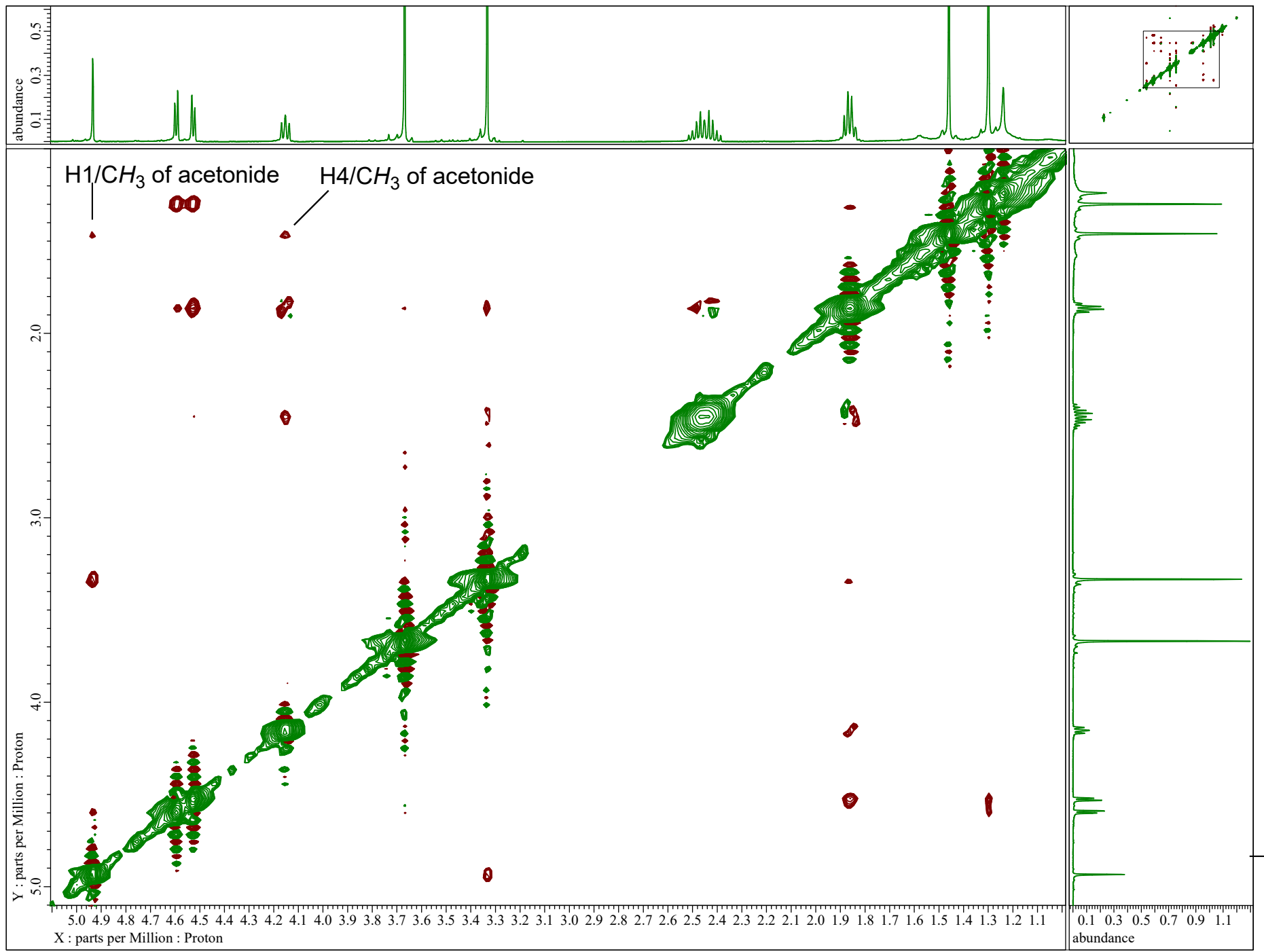




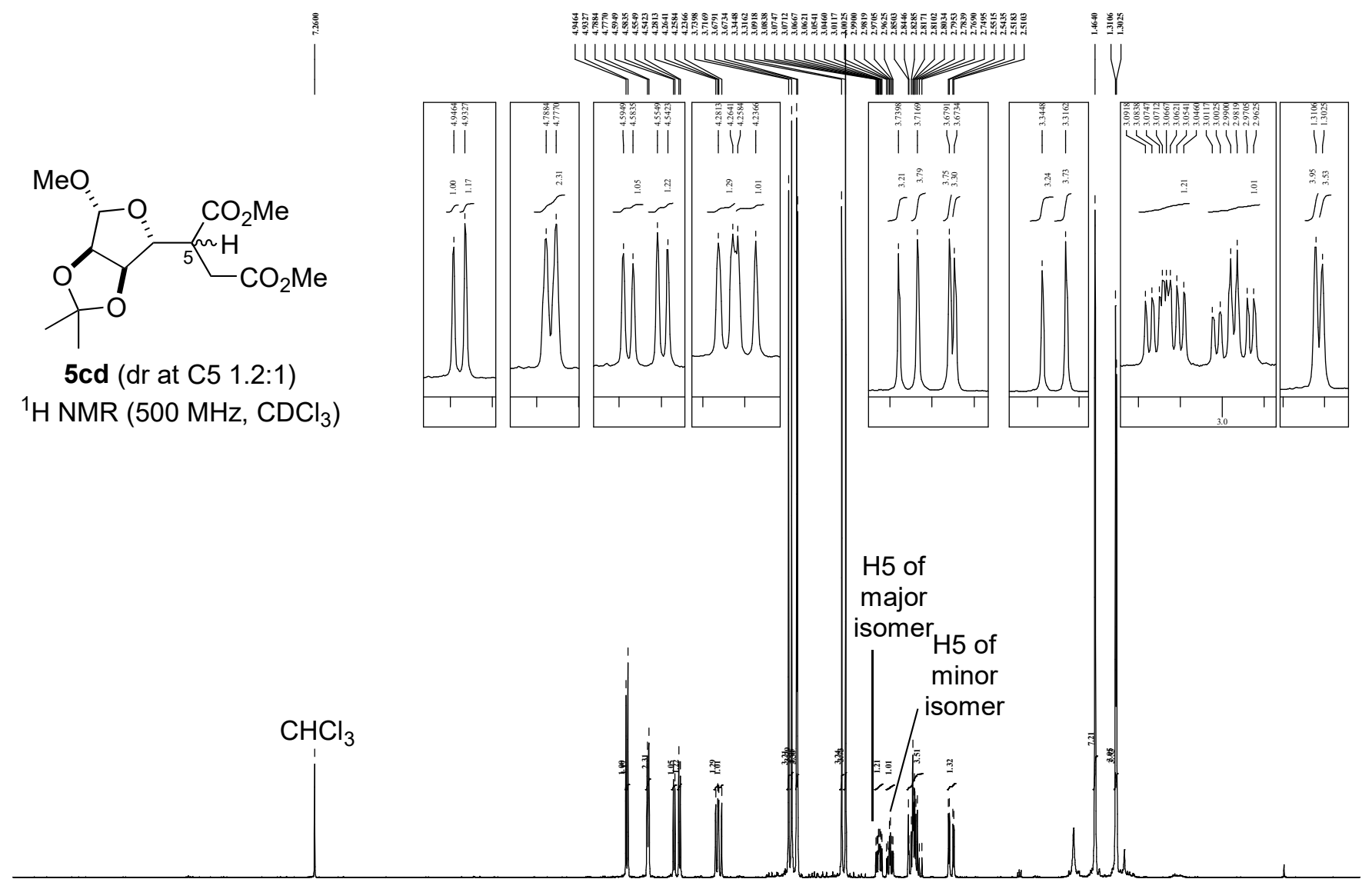

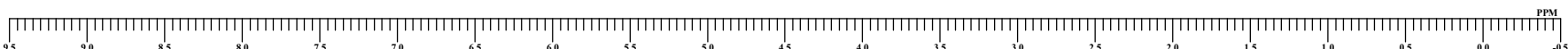

$\mathrm{MeO}$<smiles>COCC(CC(C)=O)(CC(C)=O)[C@H]1OC[C@H]2OC(C)(C)OC21</smiles>

$\mathbf{5 c d}(\mathrm{dr}$ at C5 1.2:1)

${ }^{13} \mathrm{C}\left\{{ }^{1} \mathrm{H}\right\}$ NMR $\left(125 \mathrm{MHz}, \mathrm{CDCl}_{3}\right)$

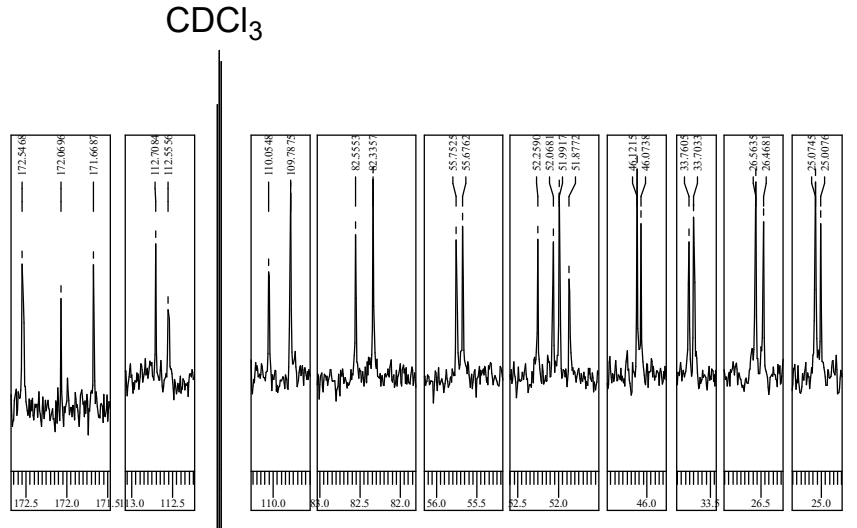

100.0

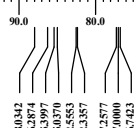

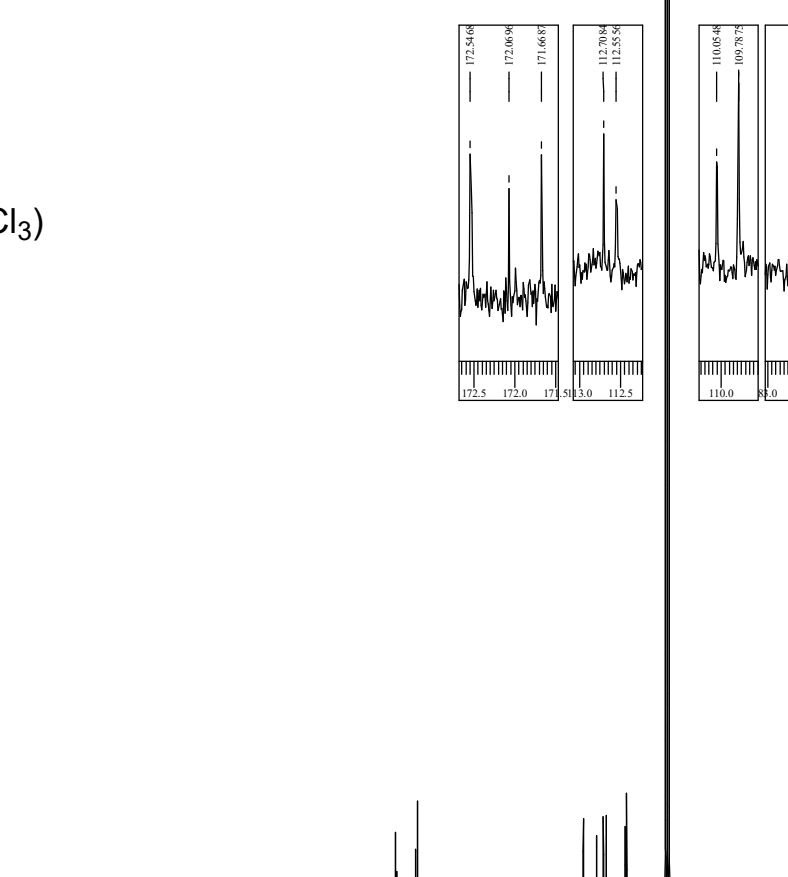

$\mathrm{MeO}$

$\mathrm{MeO}$

${ }^{1} \mathrm{H}$ NMR $\left(500 \mathrm{MHz}, \mathrm{CDCl}_{3}\right.$ )

\section{PPM}




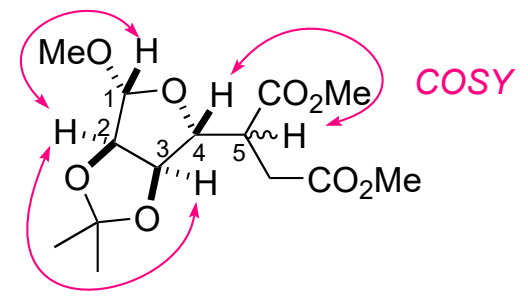

5cd (dr at C5 1.2:1)

$\operatorname{cosY}\left(500 \mathrm{MHz}, \mathrm{CDCl}_{3}\right)$

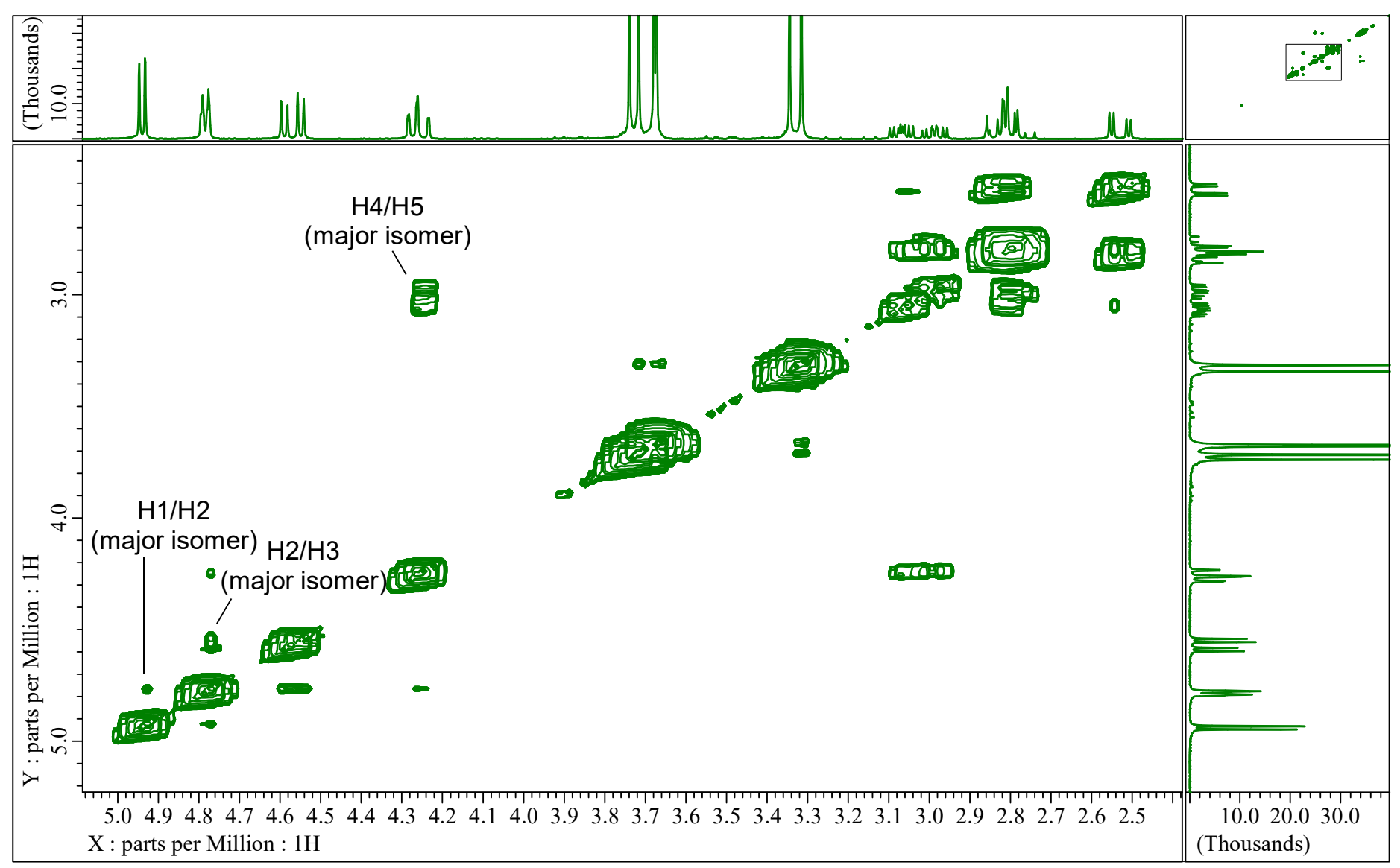




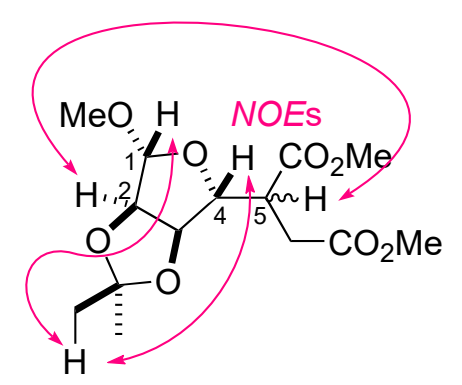

$5 \mathbf{c d}(\mathrm{dr}$ at C5 1.2:1)

NOESY $\left(500 \mathrm{MHz}, \mathrm{CDCl}_{3}\right)$

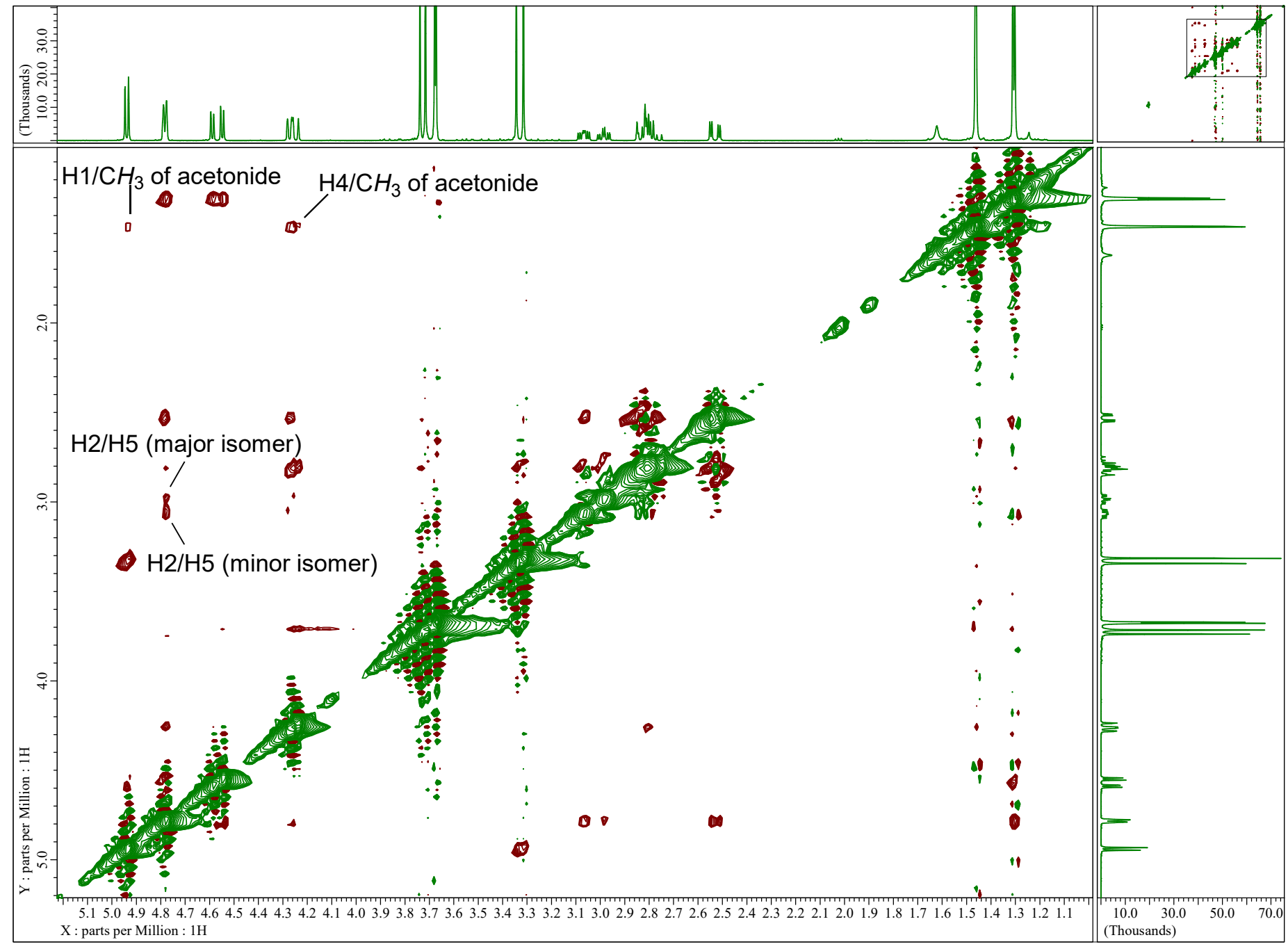




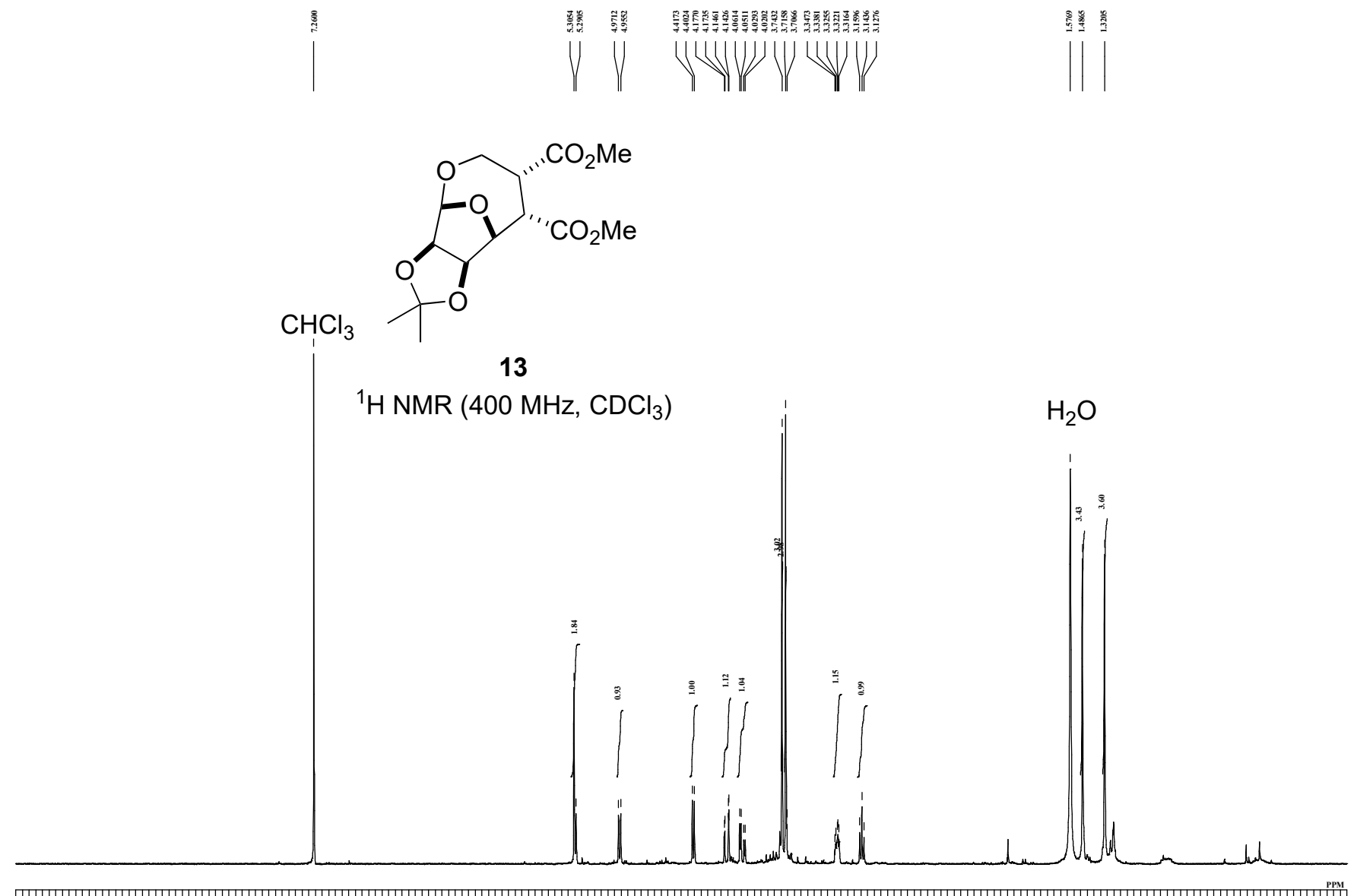

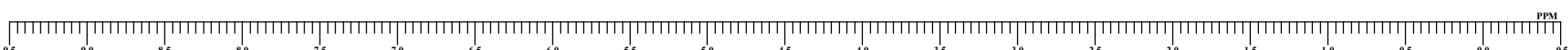

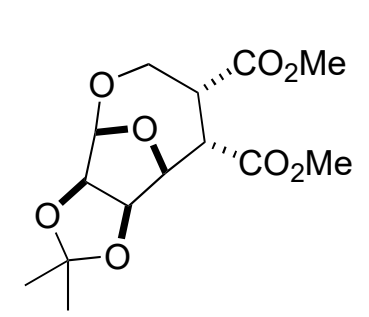

$$
\mathrm{CDCl}_{3}
$$

13

${ }^{13} \mathrm{C}\left\{{ }^{1} \mathrm{H}\right\} \mathrm{NMR}\left(100 \mathrm{MHz}, \mathrm{CDCl}_{3}\right)$

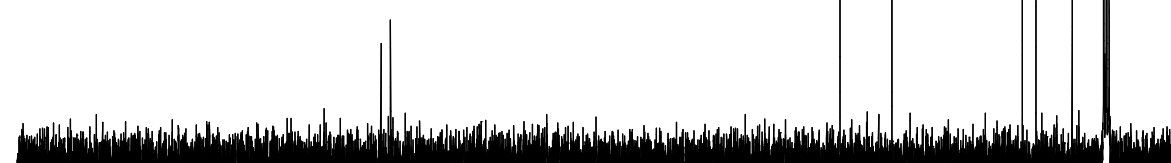

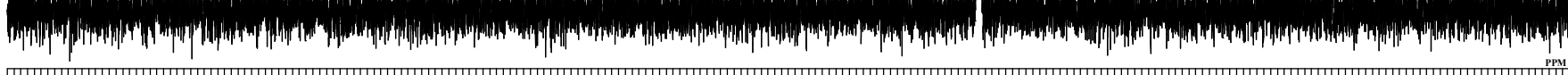

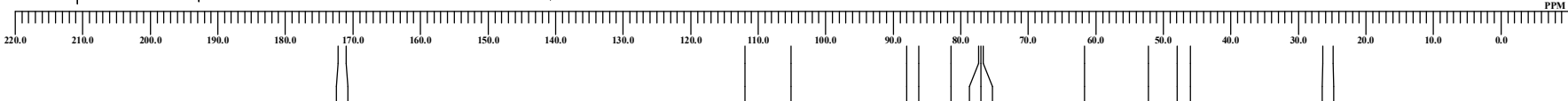




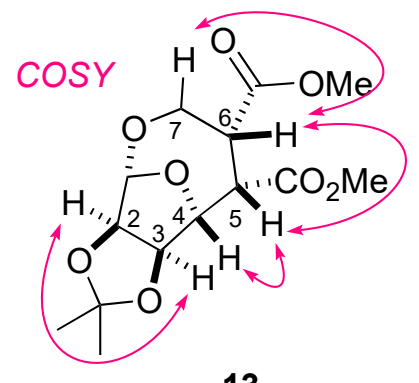

13

$\operatorname{cosy}\left(400 \mathrm{MHz}, \mathrm{CDCl}_{3}\right)$

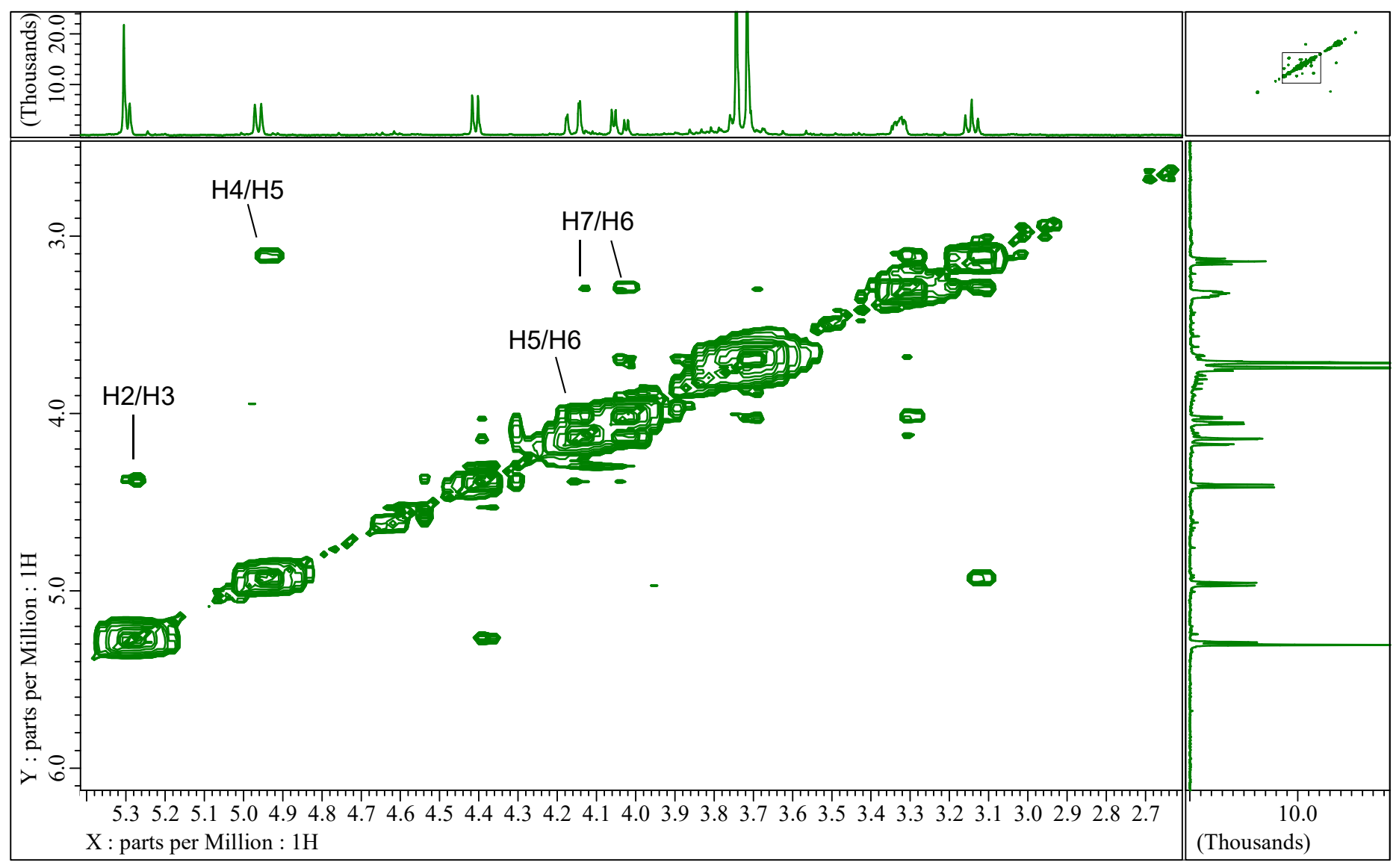




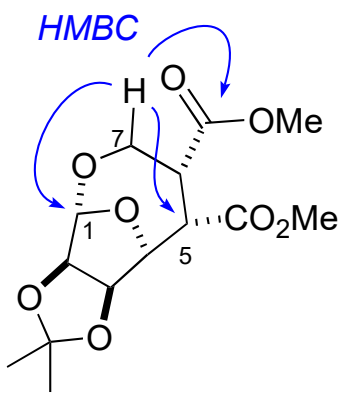

13

$\mathrm{HMBC}\left(400 \mathrm{MHz}, \mathrm{CDCl}_{3}\right)$

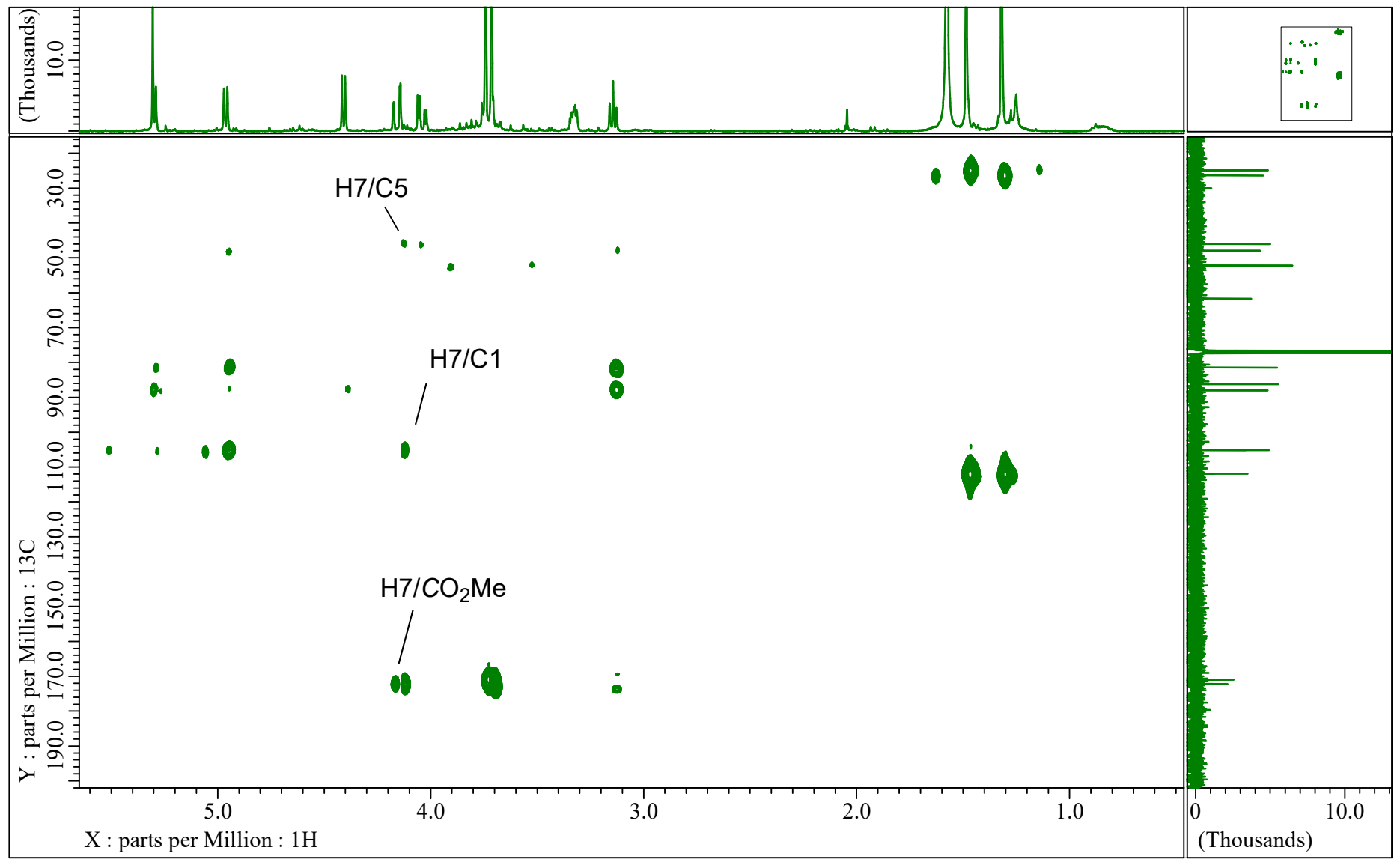



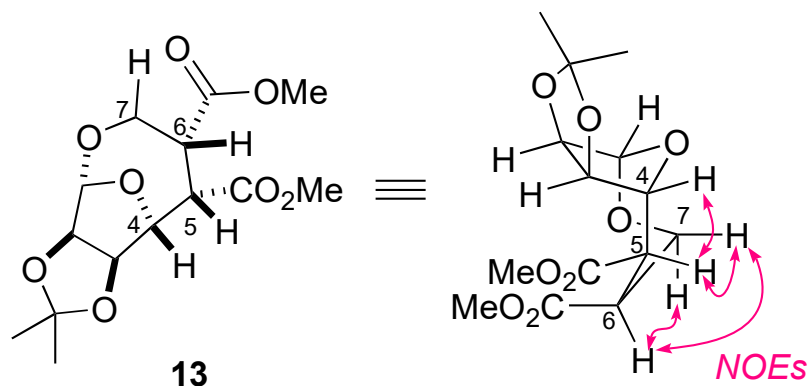

NOESY $\left(400 \mathrm{MHz}, \mathrm{CDCl}_{3}\right)$

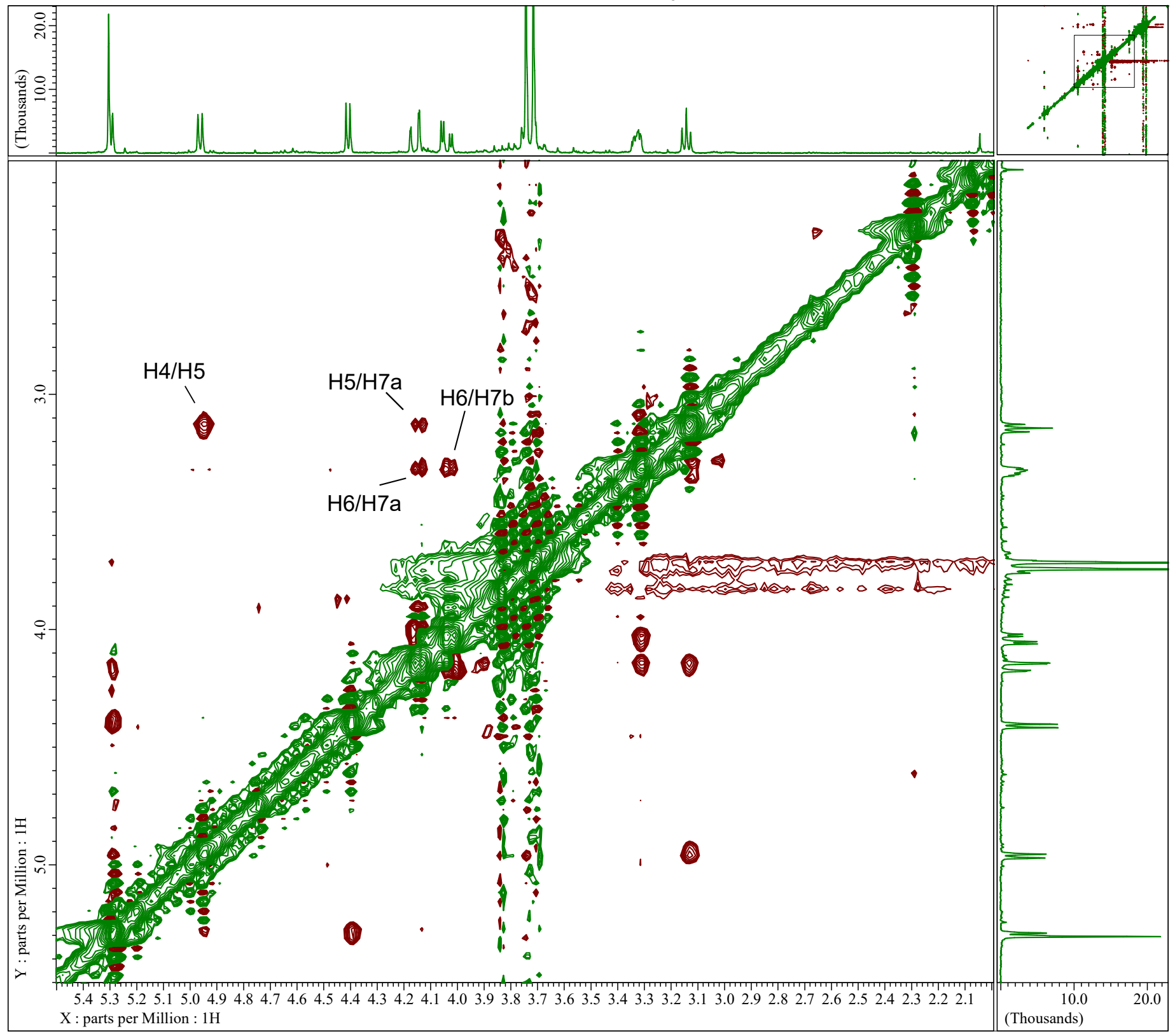




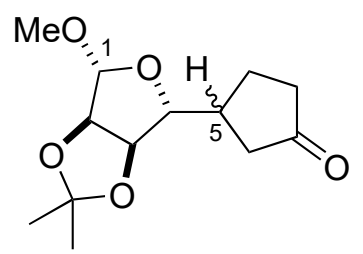

5cf (dr at C5 2:1)

${ }^{1} \mathrm{H}$ NMR $\left(500 \mathrm{MHz}, \mathrm{CDCl}_{3}\right)$

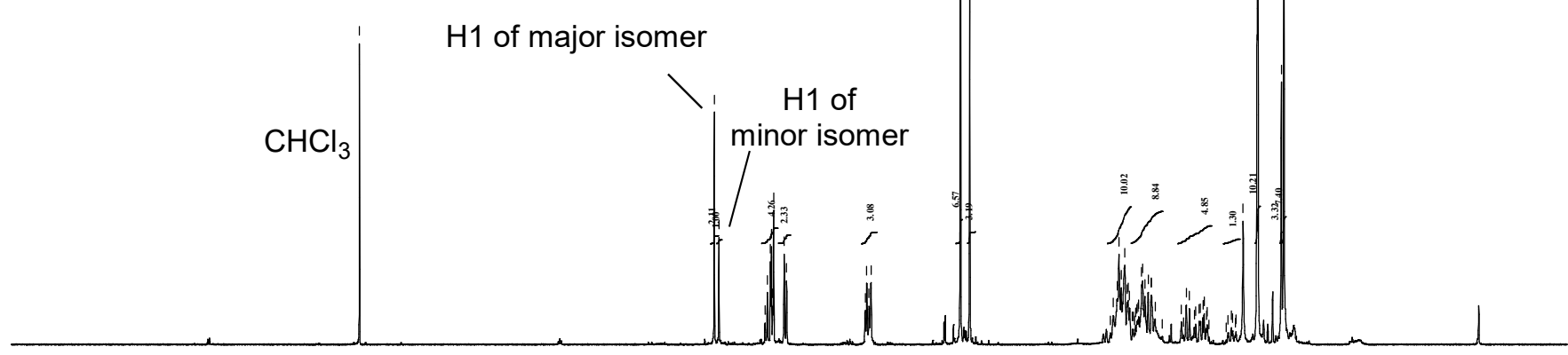

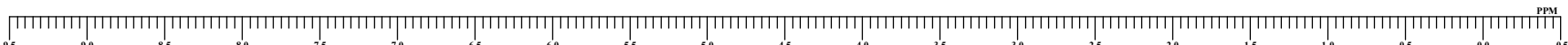


$\mathrm{MeO}$

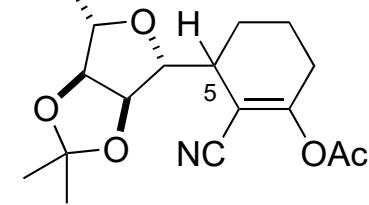

5cg-Ac (major isomer)

${ }^{1} \mathrm{H}$ NMR $\left(400 \mathrm{MHz}, \mathrm{CDCl}_{3}\right)$

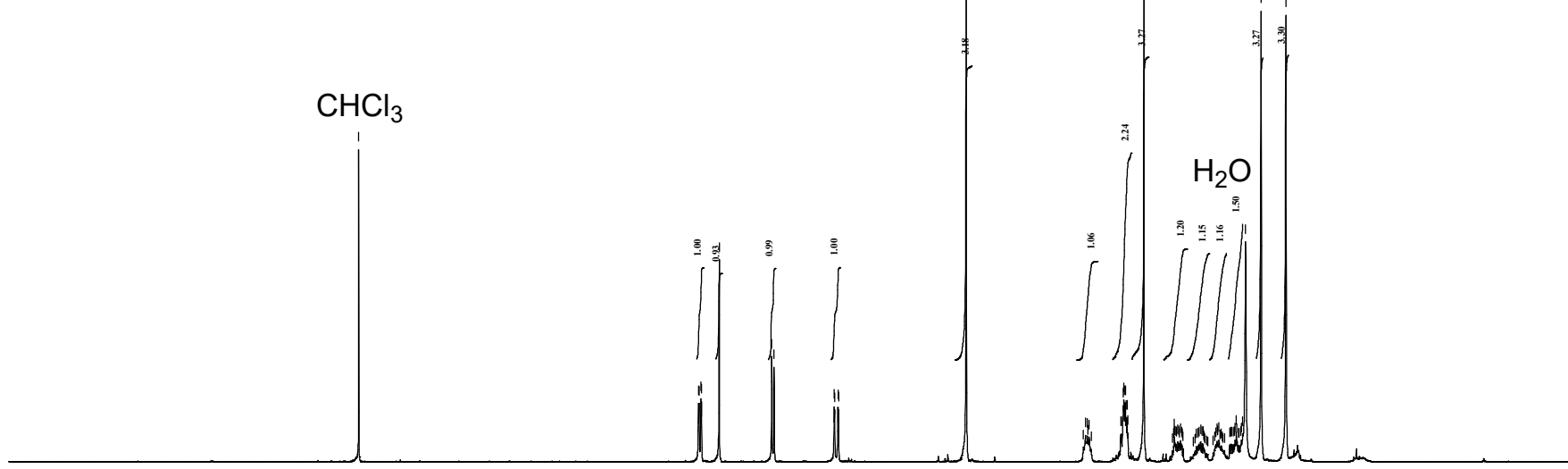

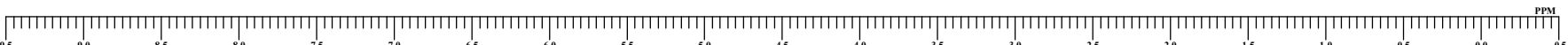

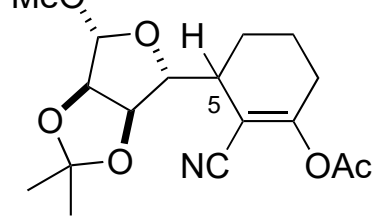

5cg-Ac (major isomer)

${ }^{13} \mathrm{C}\left\{{ }^{1} \mathrm{H}\right\} \mathrm{NMR}\left(100 \mathrm{MHz}, \mathrm{CDCl}_{3}\right)$

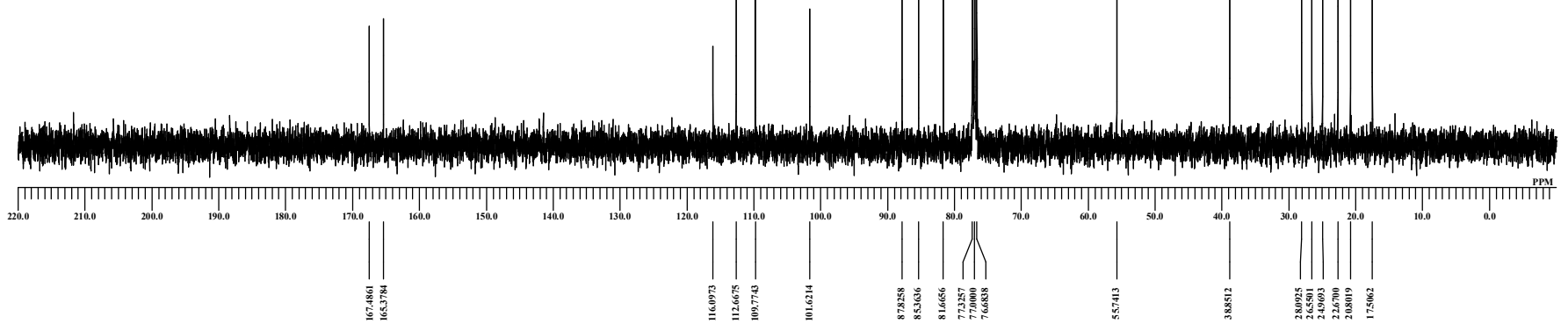




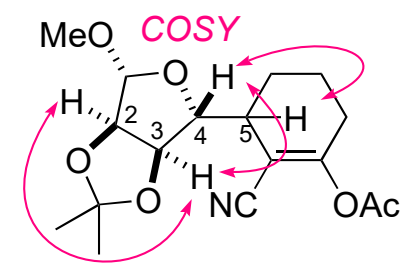

major isomer of $\mathbf{5 c g}-\mathbf{A c}$

$\operatorname{cosY}\left(400 \mathrm{MHz}, \mathrm{CDCl}_{3}\right)$

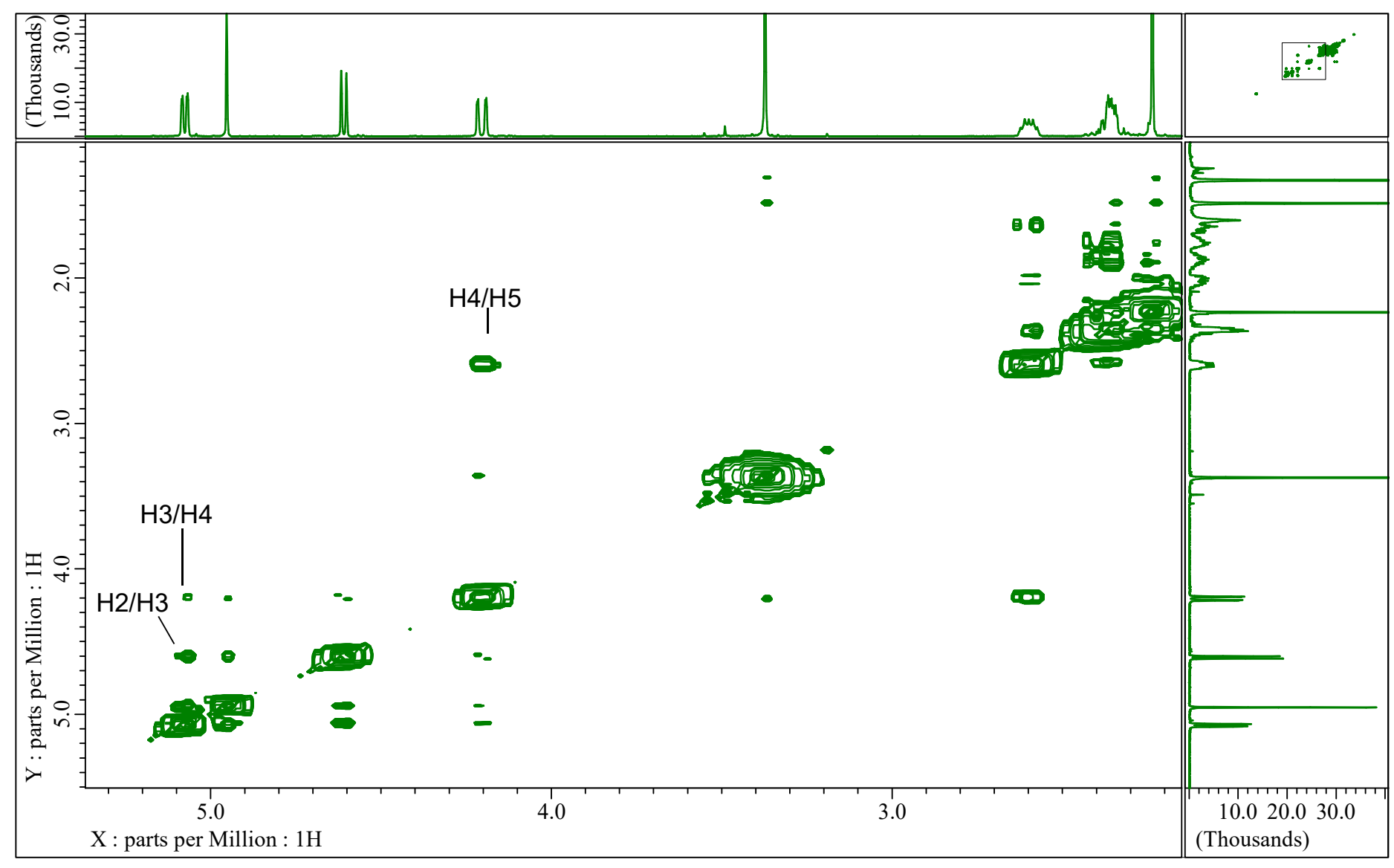




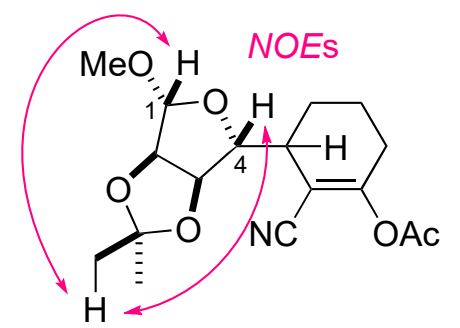

major isomer of $\mathbf{5 c g}-\mathbf{A c}$

$\operatorname{NOESY}\left(400 \mathrm{MHz}, \mathrm{CDCl}_{3}\right)$

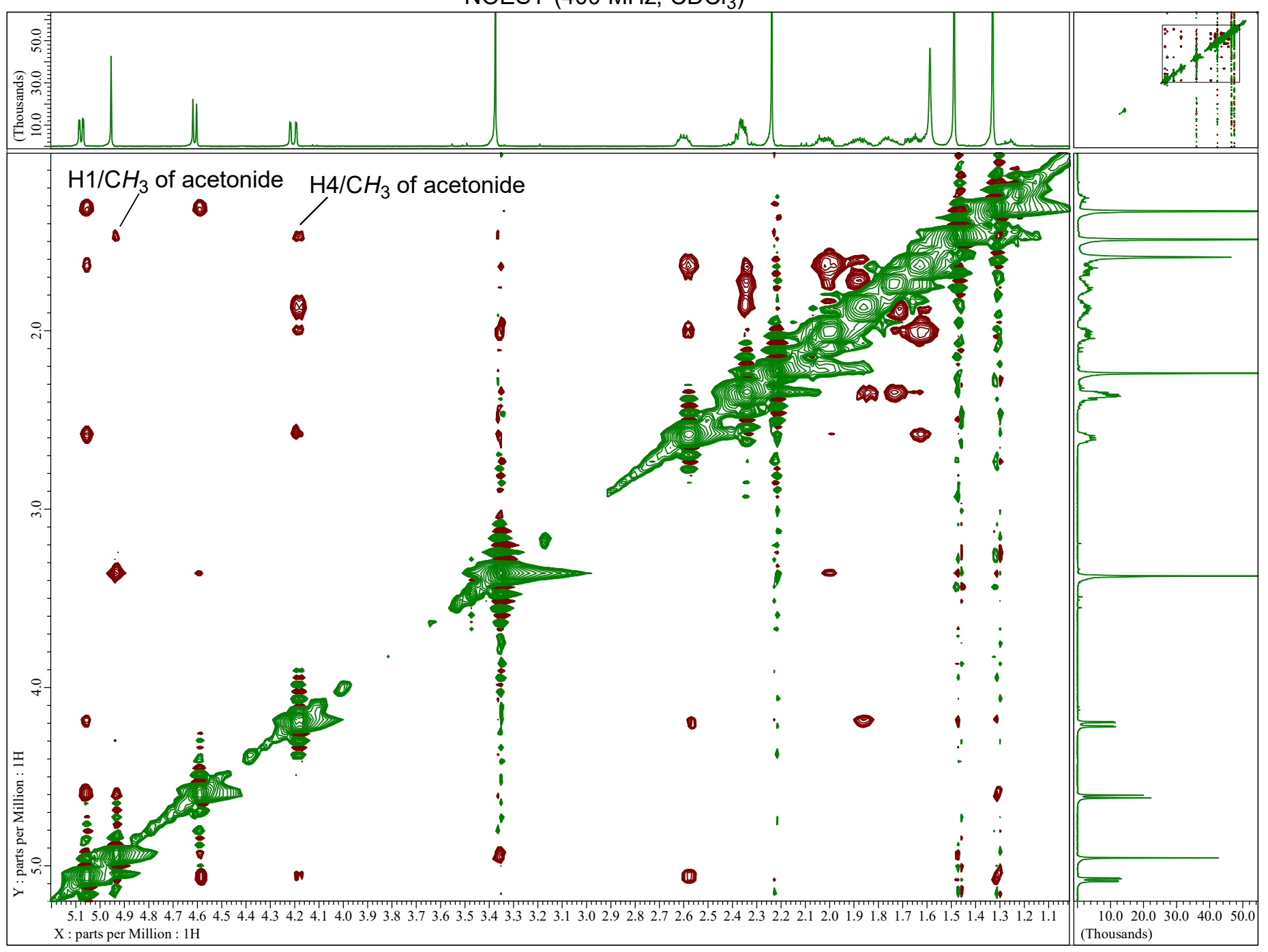


$\mathrm{MeO}$

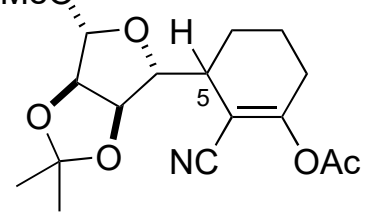

$\mathbf{5 c g}-\mathrm{Ac}$ (minor isomer)

${ }^{1} \mathrm{H}$ NMR (400 $\left.\mathrm{MHz}, \mathrm{CDCl}_{3}\right)$

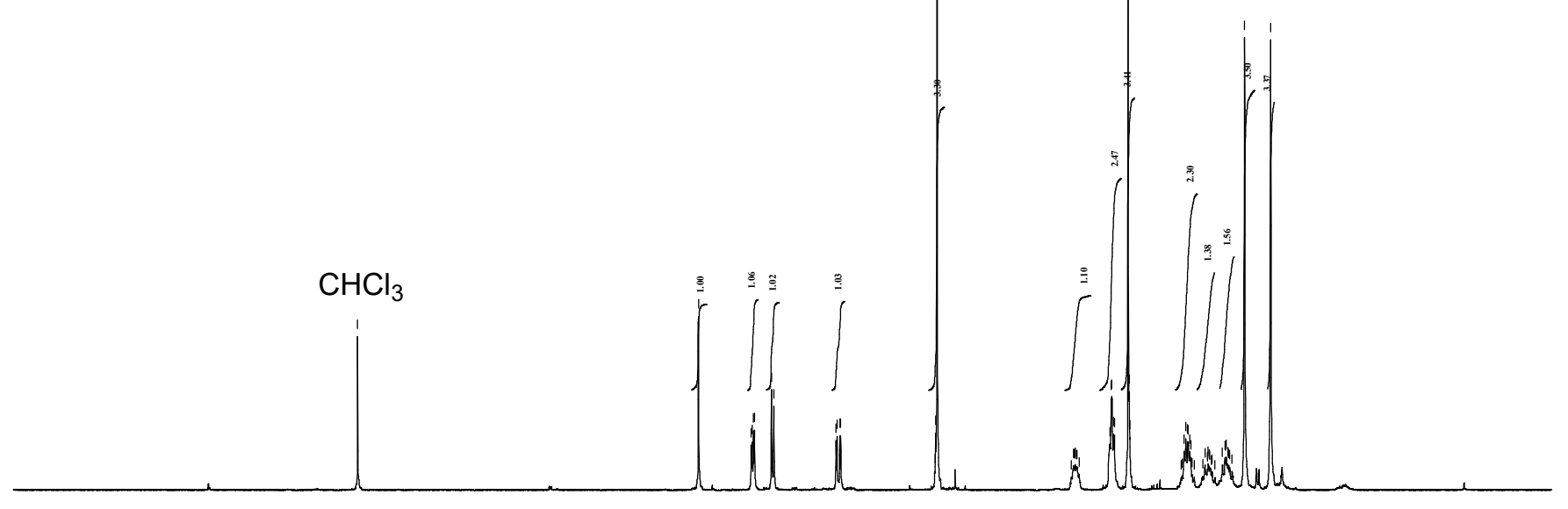

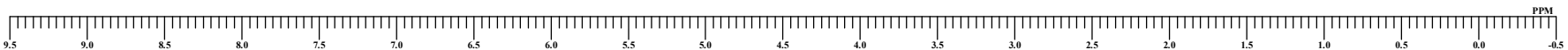

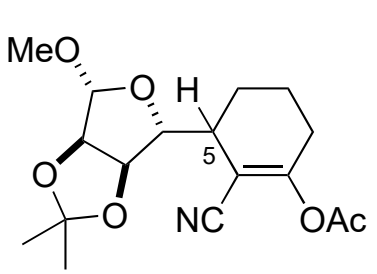

5cg-Ac (minor isomer)

${ }^{13} \mathrm{C}\left\{{ }^{1} \mathrm{H}\right\} \operatorname{NMR}\left(100 \mathrm{MHz}, \mathrm{CDCl}_{3}\right)$

\section{$\mathrm{CDCl}_{3}$}

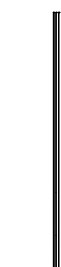




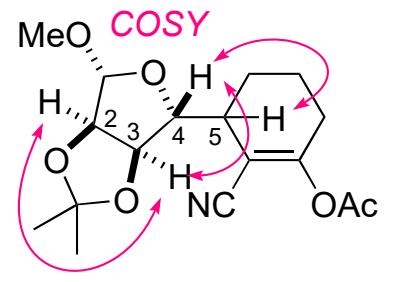

minor isomer of $\mathbf{5 c g}-\mathbf{A c}$

$\operatorname{COSY}\left(500 \mathrm{MHz}, \mathrm{CDCl}_{3}\right)$

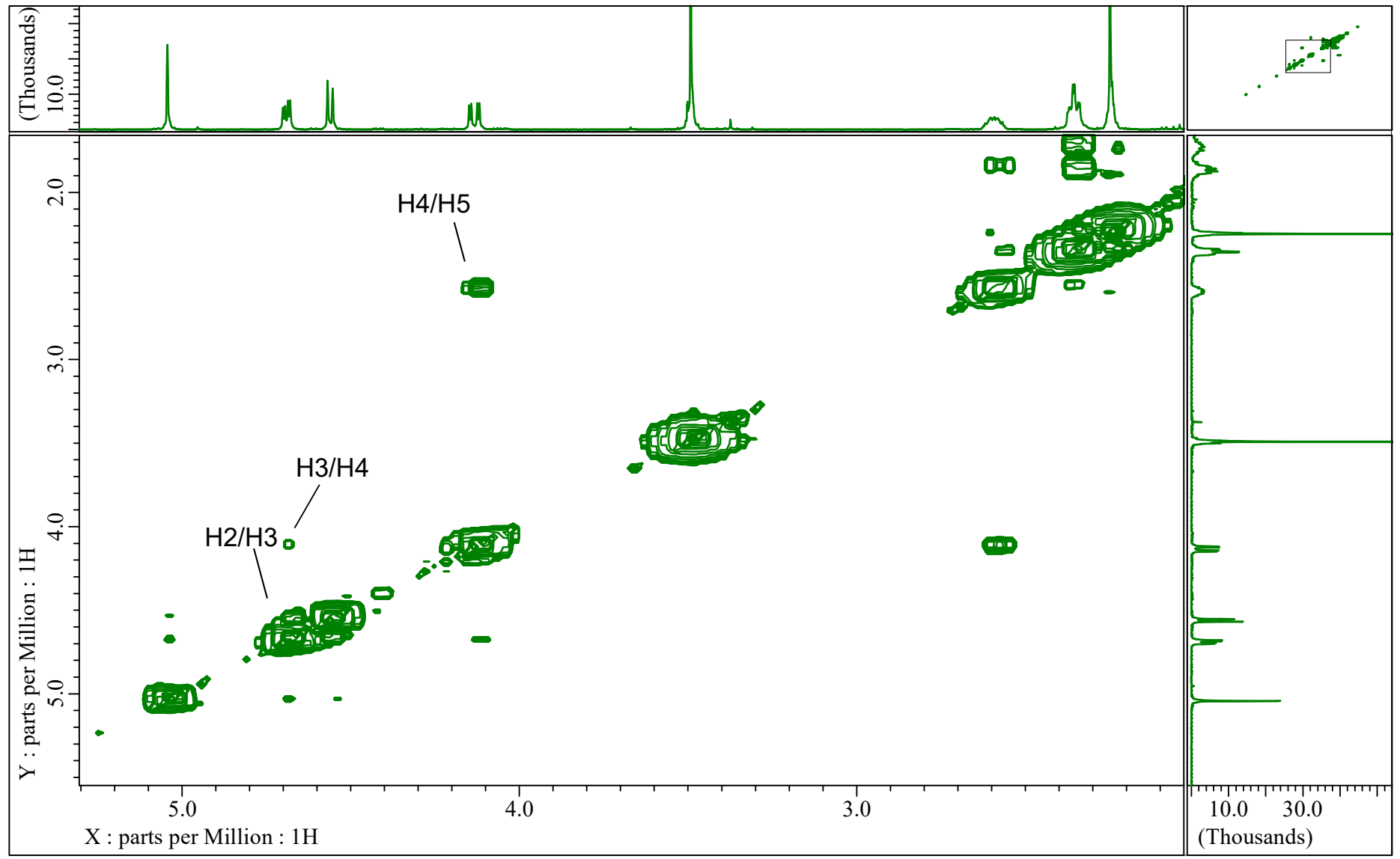




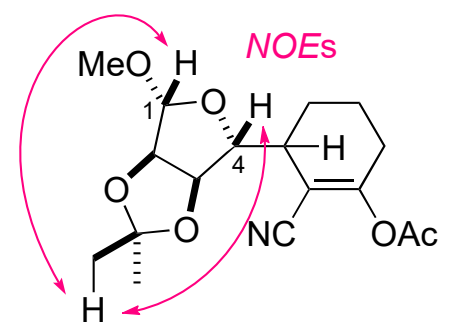

minor isomer of $\mathbf{5 c g}-\mathbf{A c}$

NOESY $\left(500 \mathrm{MHz}, \mathrm{CDCl}_{3}\right)$

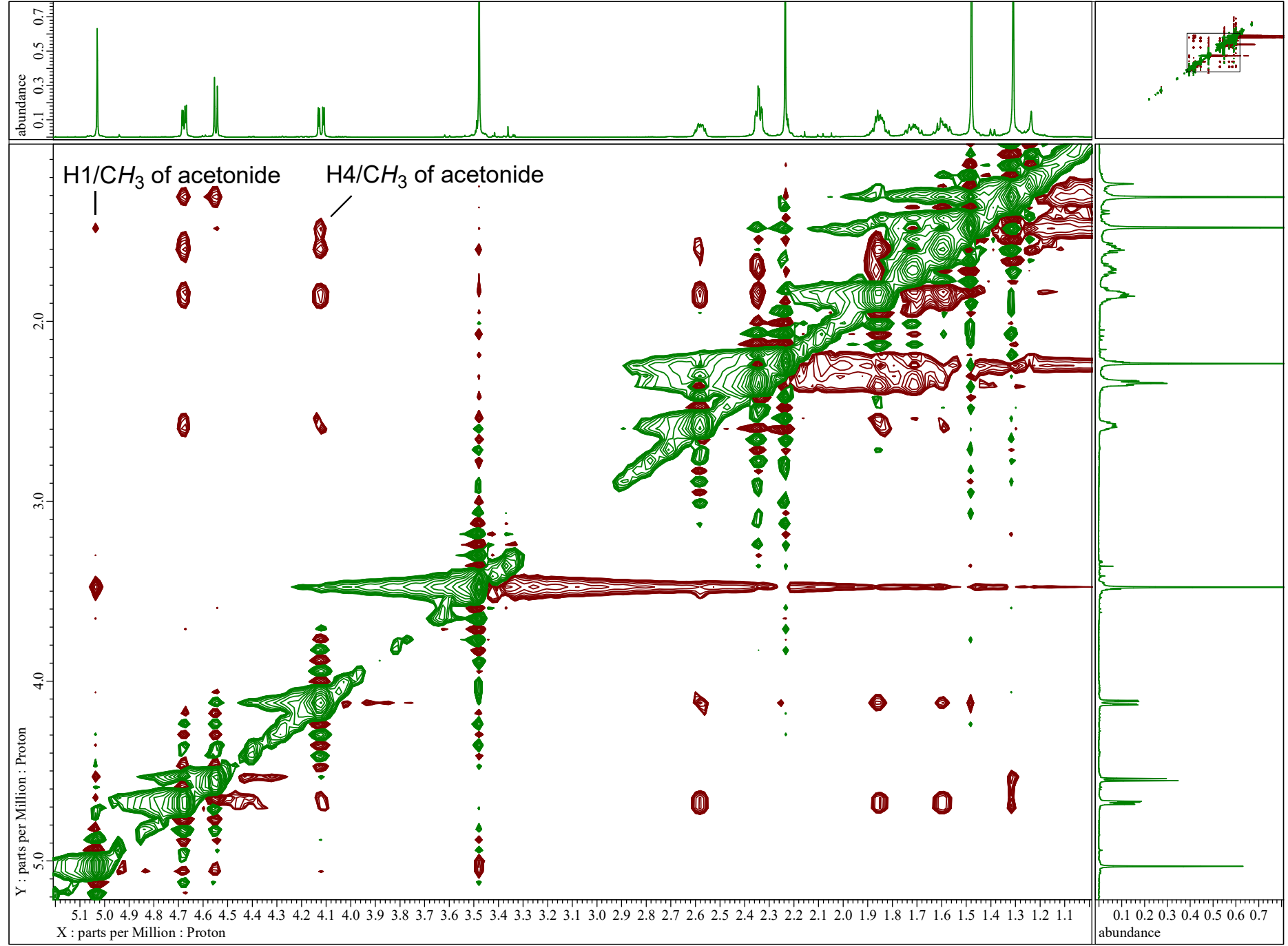




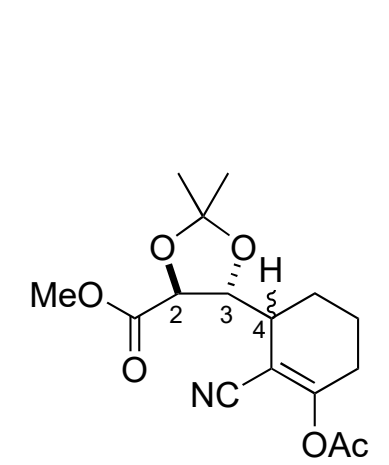

5fg-Ac (dr at C4 1.3:1)

${ }^{1} \mathrm{H} \mathrm{NMR}\left(400 \mathrm{MHz}, \mathrm{CDCl}_{3}\right)$

$\mathrm{CHCl}_{3}$
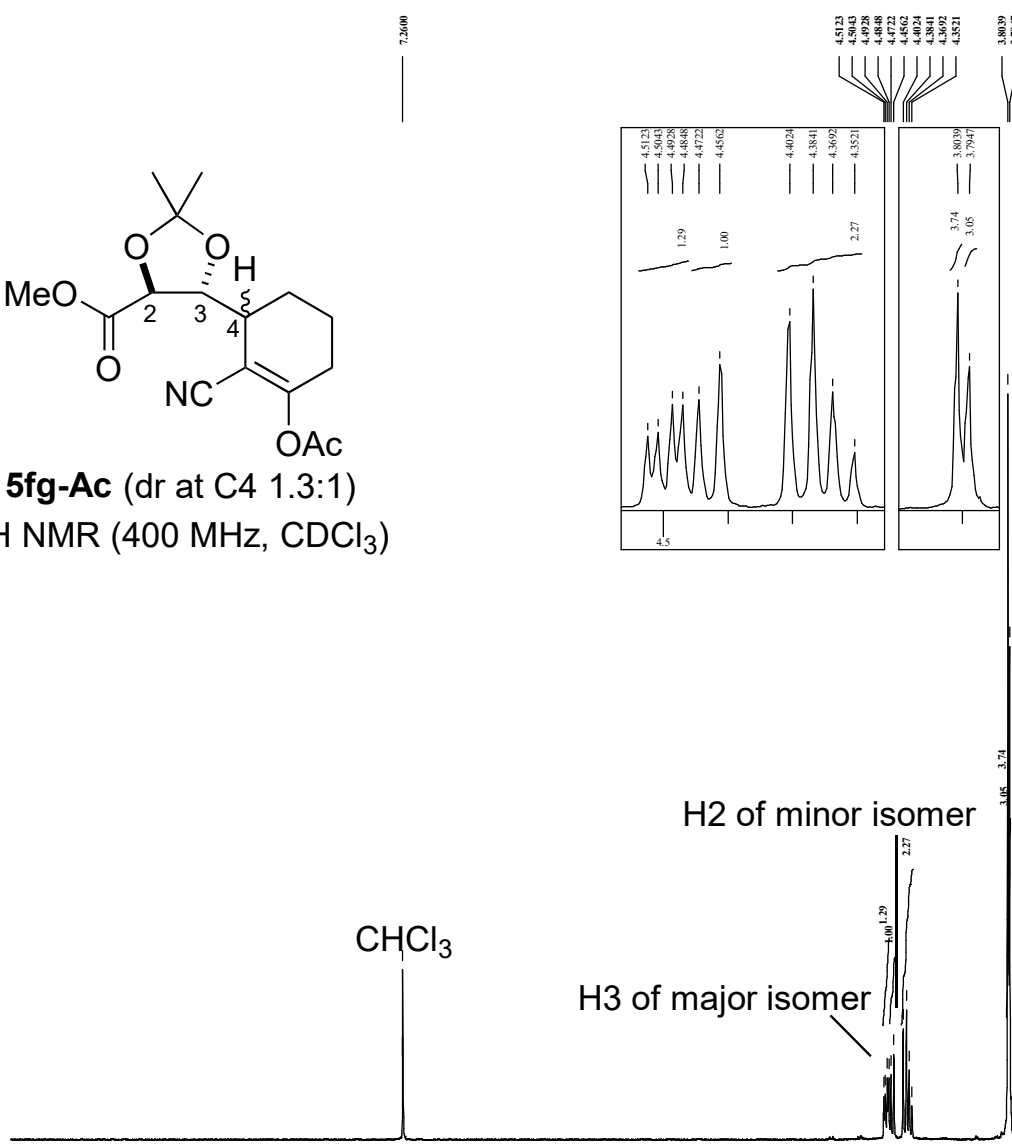

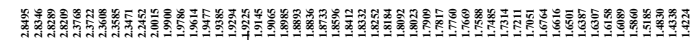

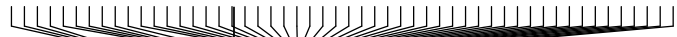
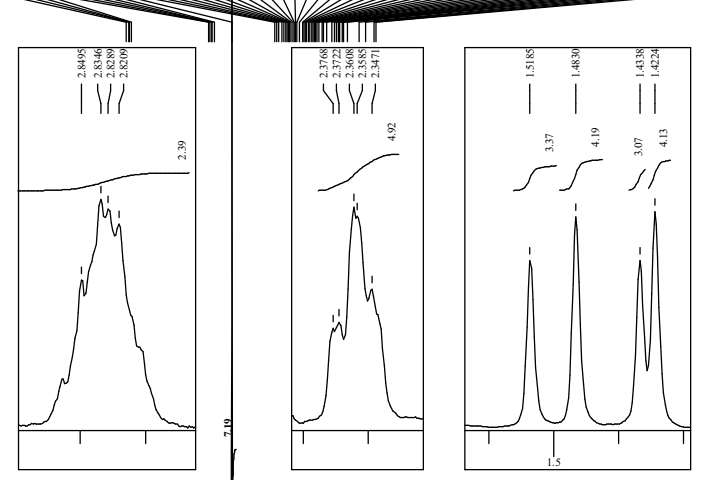

H2 of minor isomer

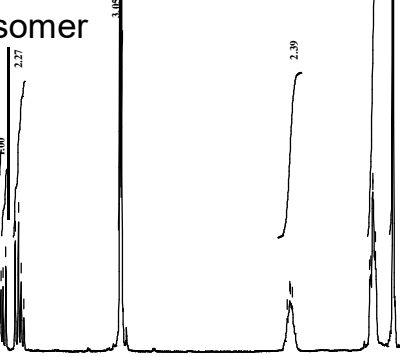

(n)

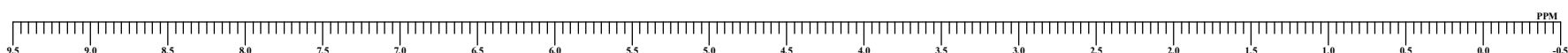<smiles>COC(=O)C1OC(C)(C)O[C@H]1C1CCCC(OC(C)=O)=C1C#N</smiles>

$\mathrm{CDCl}_{3}$

5fg-Ac (dr at C4 1.3:1)

${ }^{13} \mathrm{C}\left\{{ }^{1} \mathrm{H}\right\}$ NMR $\left(100 \mathrm{MHz}, \mathrm{CDCl}_{3}\right)$

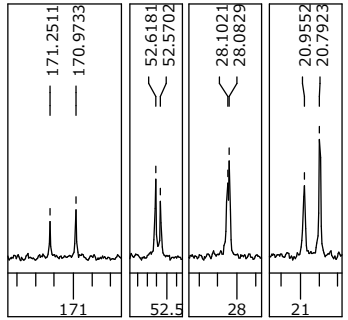

|

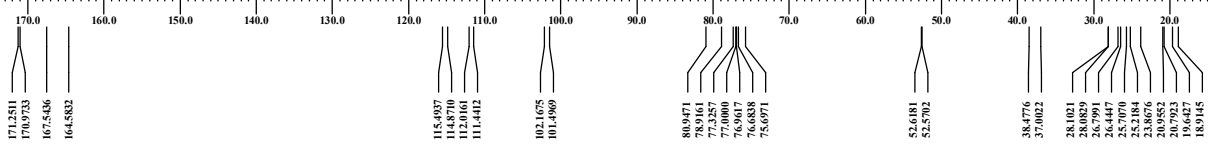




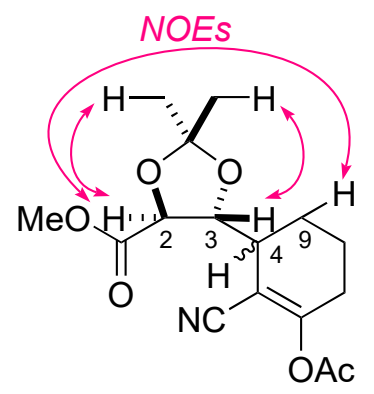

5fg-Ac (dr at C4 1.3:1) NOESY $\left(500 \mathrm{MHz}, \mathrm{CDCl}_{3}\right)$

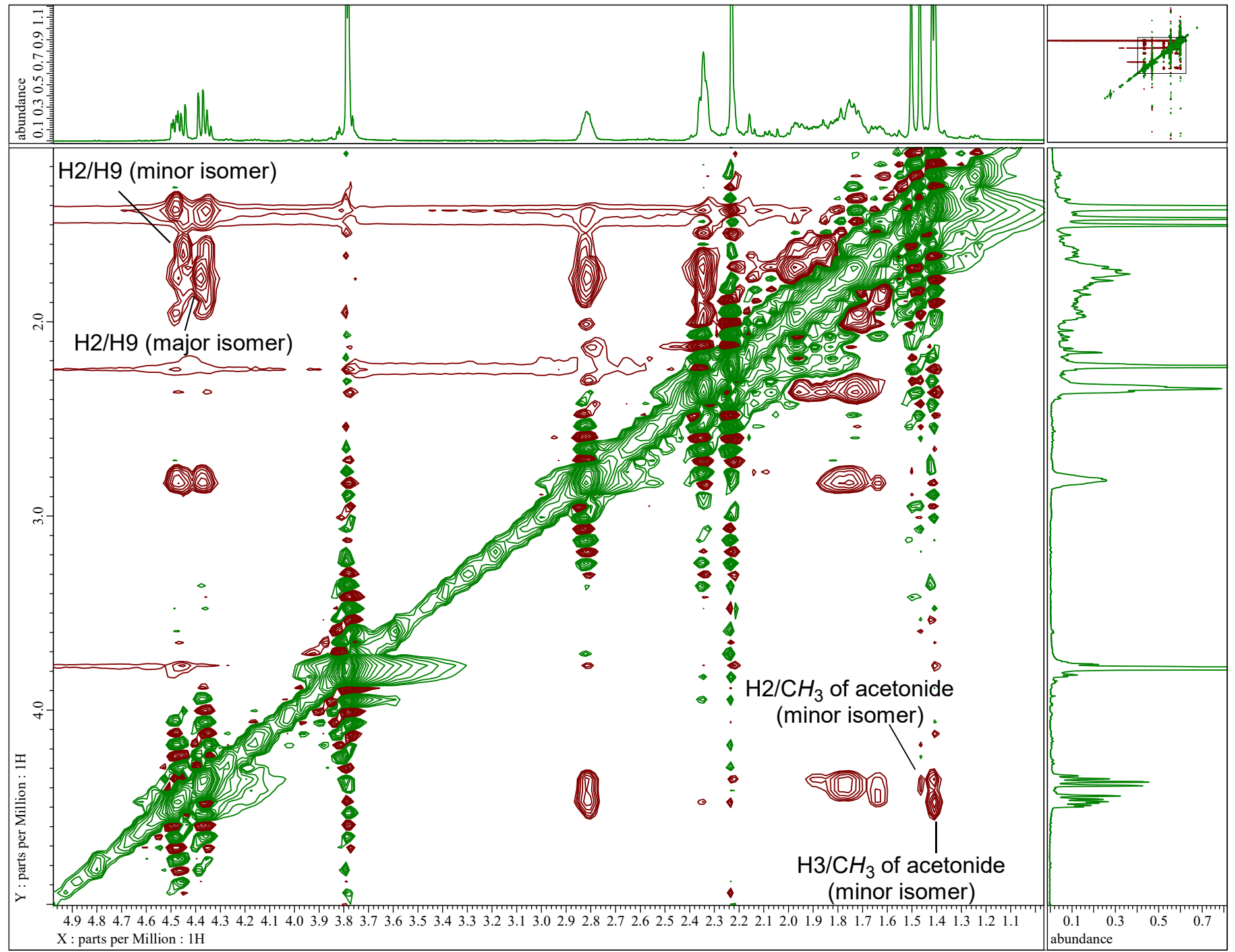


<smiles>CC(=O)OC1=C(C#N)C(C23CC4CC(C2)OC(C)(O4)O3)CCC1</smiles>

\section{5gg-Ac}

${ }^{1} \mathrm{H}$ NMR (400 MHz, $\left.\mathrm{CDCl}_{3}\right)$

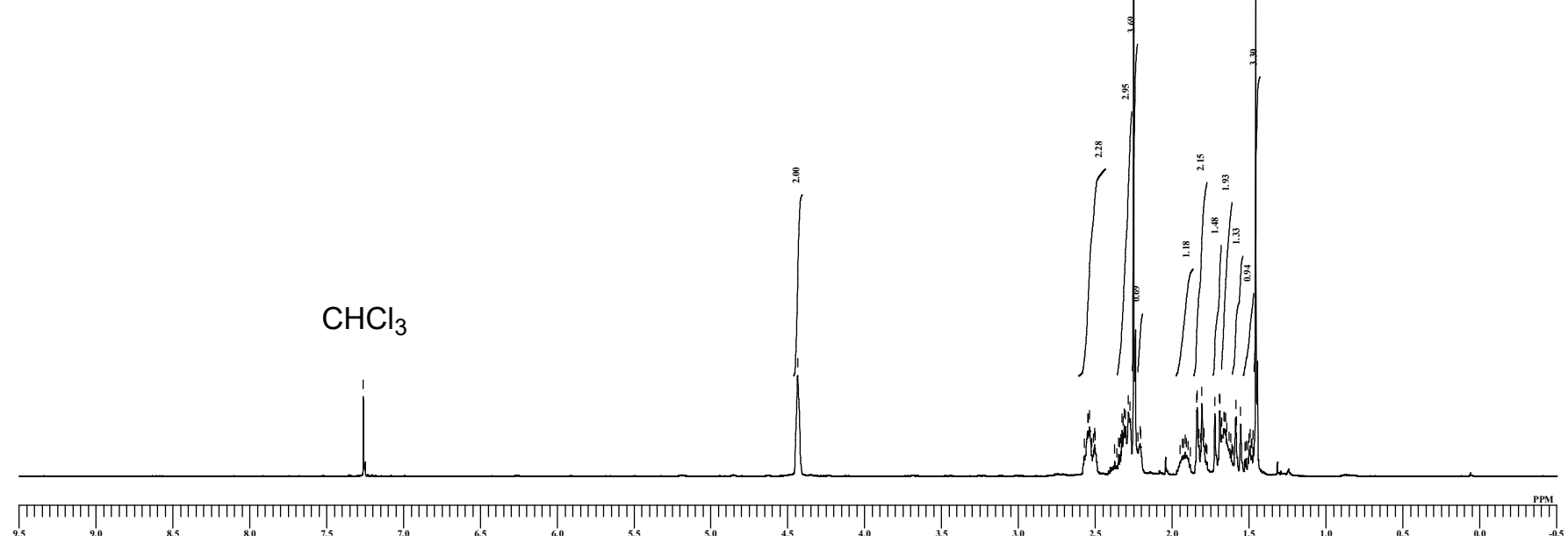<smiles>CC(=O)OC1=C(C#N)C(C23CC4CC(C2)OC(C)(O4)O3)CCC1</smiles>

5gg-Ac

${ }^{13} \mathrm{C}\left\{{ }^{1} \mathrm{H}\right\} \operatorname{NMR}\left(100 \mathrm{MHz}, \mathrm{CDCl}_{3}\right)$

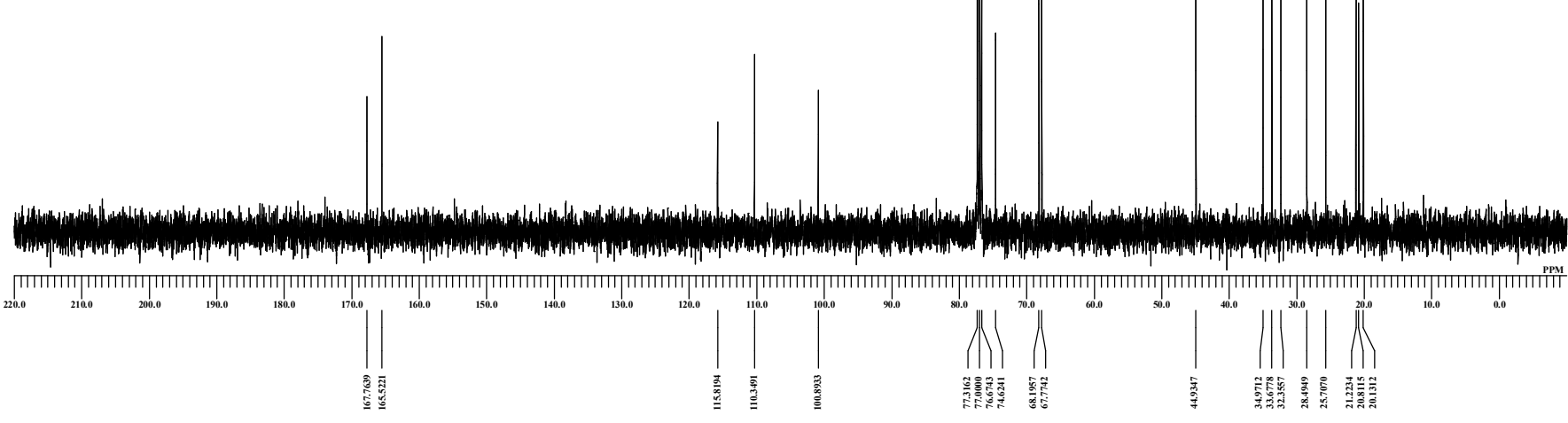




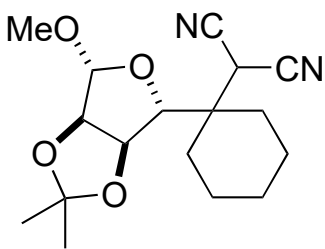

$5 \mathrm{ch}$

${ }^{1} \mathrm{H}$ NMR (400 MHz, $\mathrm{CDCl}_{3}$ )
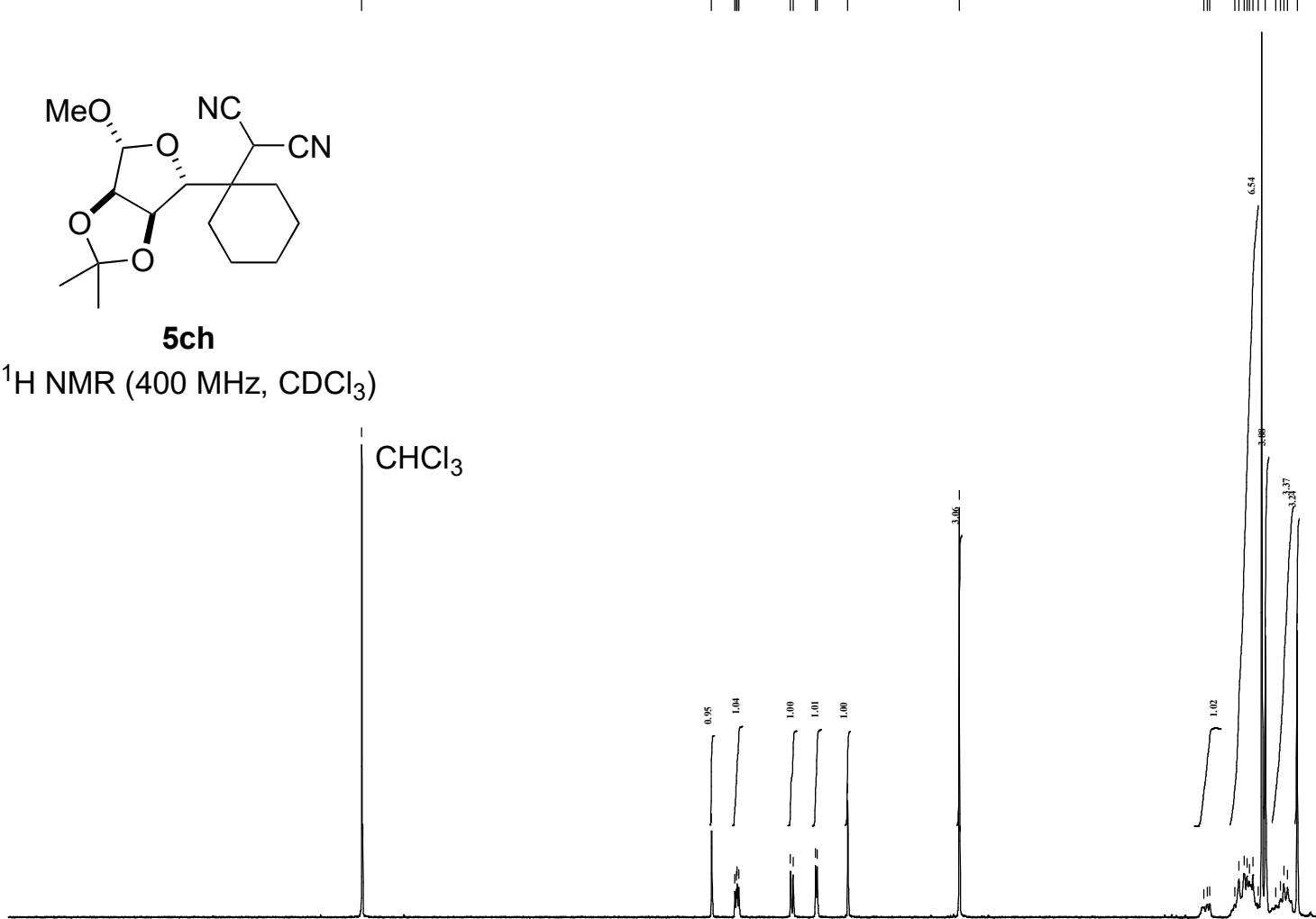

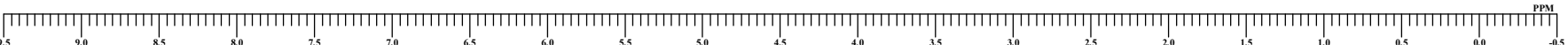

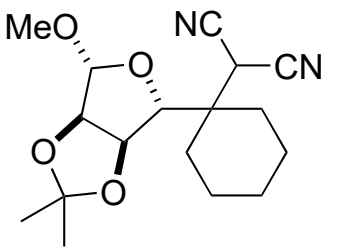

5ch

${ }^{13} \mathrm{C}\left\{{ }^{1} \mathrm{H}\right\}$ NMR $\left(100 \mathrm{MHz}, \mathrm{CDCl}_{3}\right)$
$\mathrm{CDCl}_{3}$

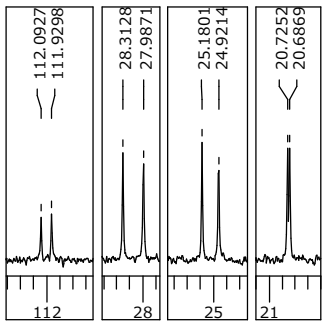

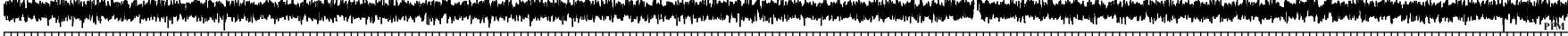
(1)

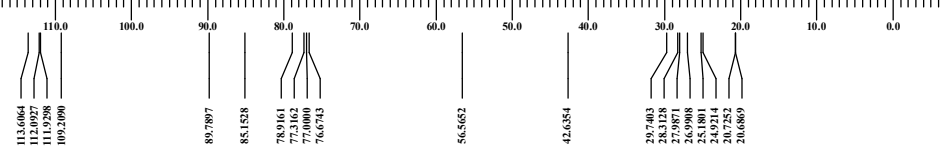




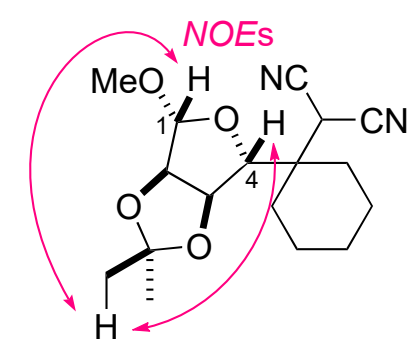

$5 \mathrm{ch}$

NOESY $\left(500 \mathrm{MHz}, \mathrm{CDCl}_{3}\right)$

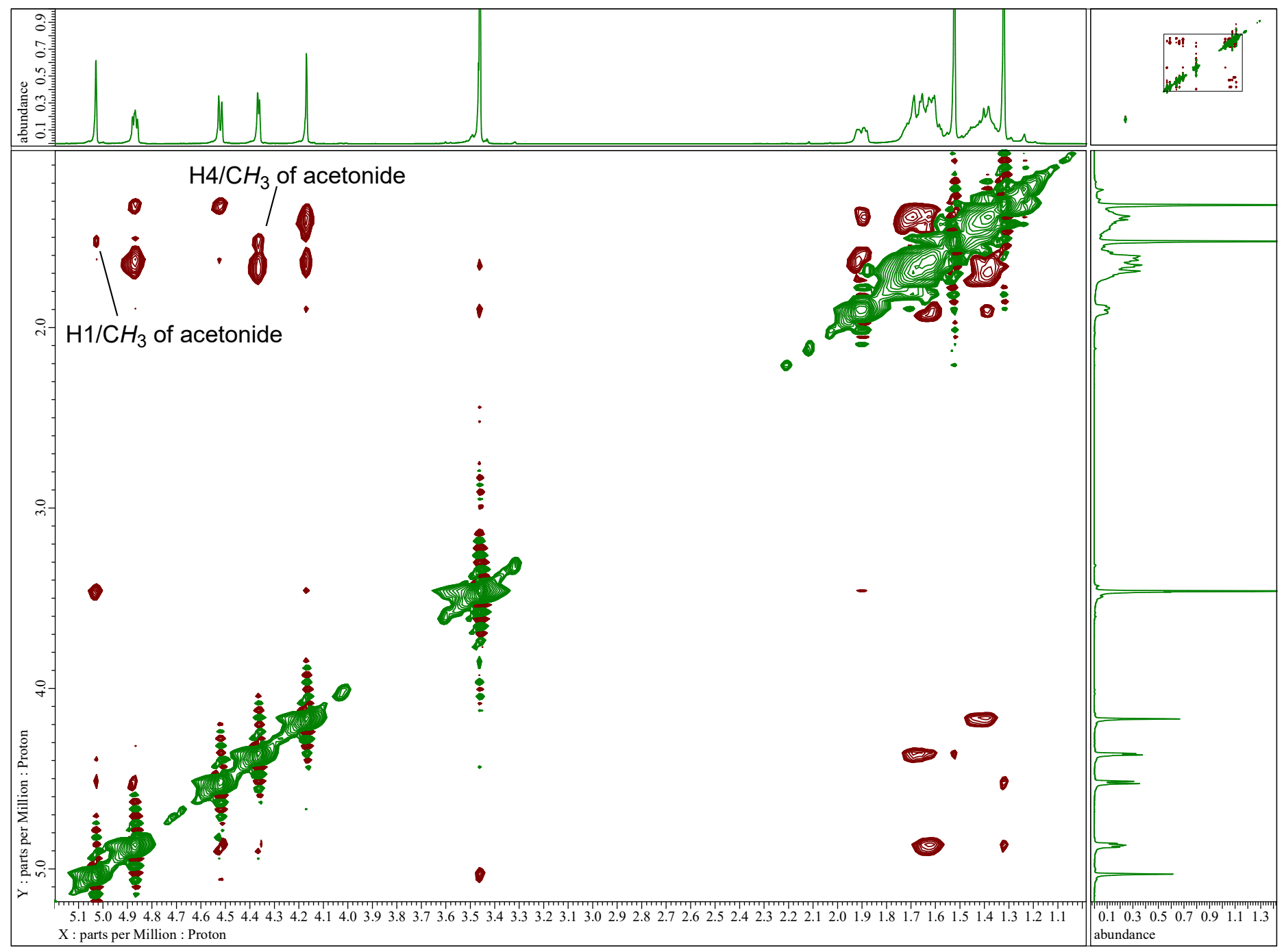




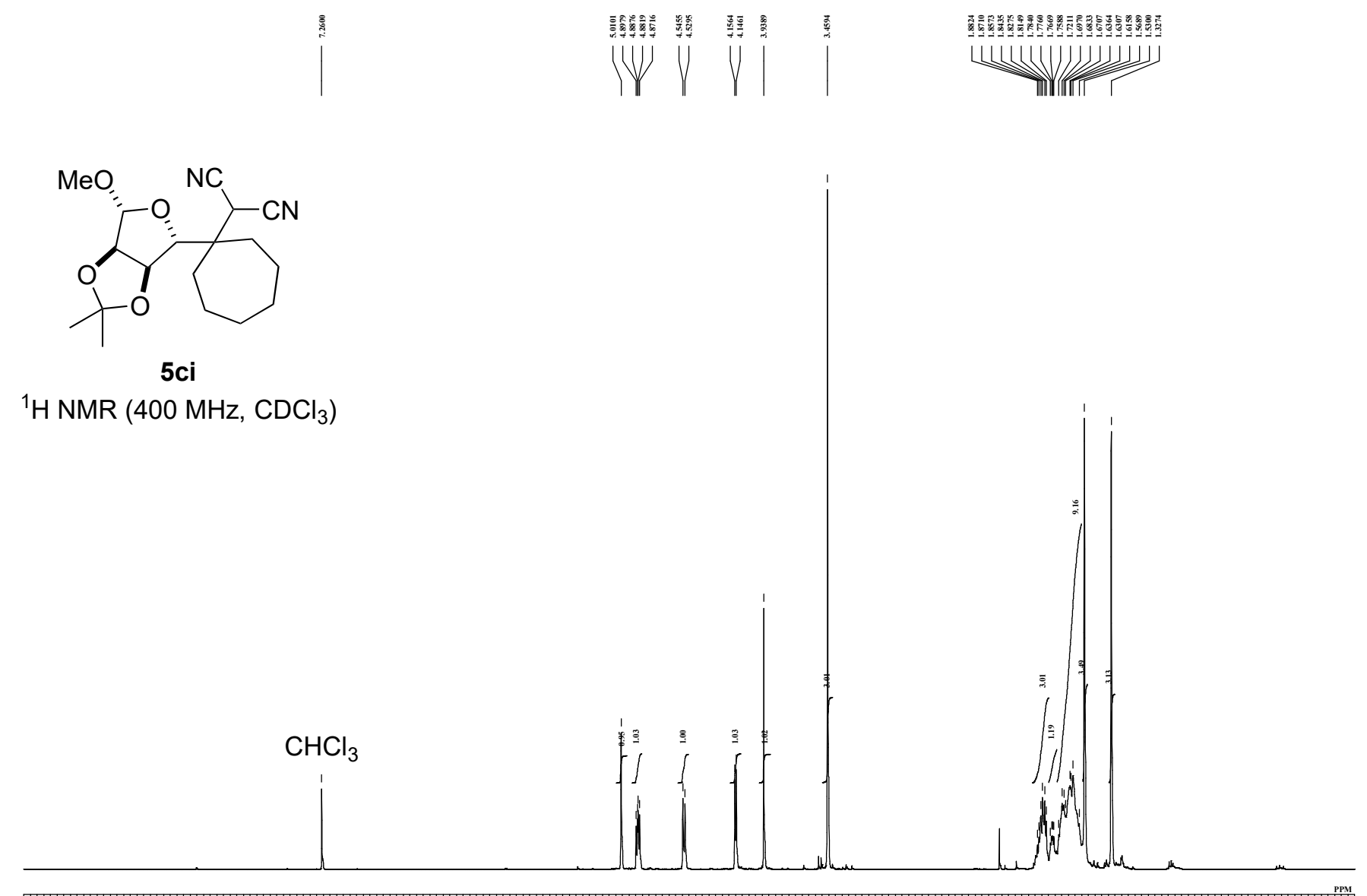

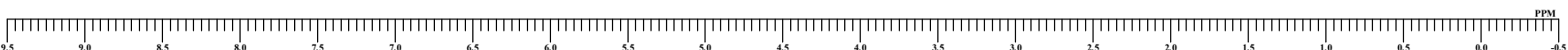

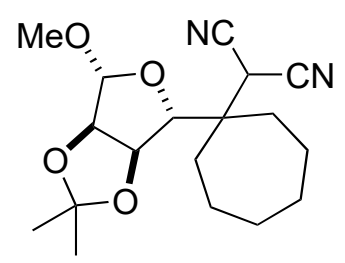

$5 \mathrm{ci}$

${ }^{13} \mathrm{C}\left\{{ }^{1} \mathrm{H}\right\} \mathrm{NMR}\left(100 \mathrm{MHz}, \mathrm{CDCl}_{3}\right)$

$\mathrm{CDCl}_{3}$

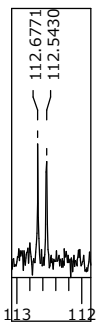
D.t.

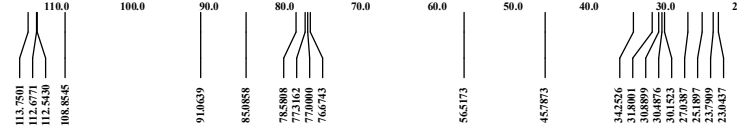




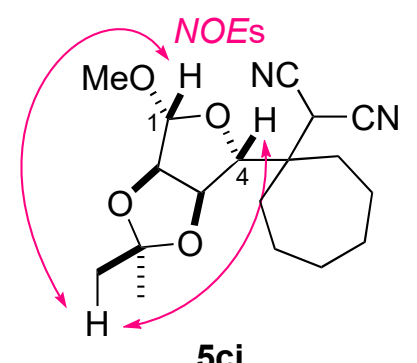

NOESY $\left(400 \mathrm{MHz}, \mathrm{CDCl}_{3}\right)$

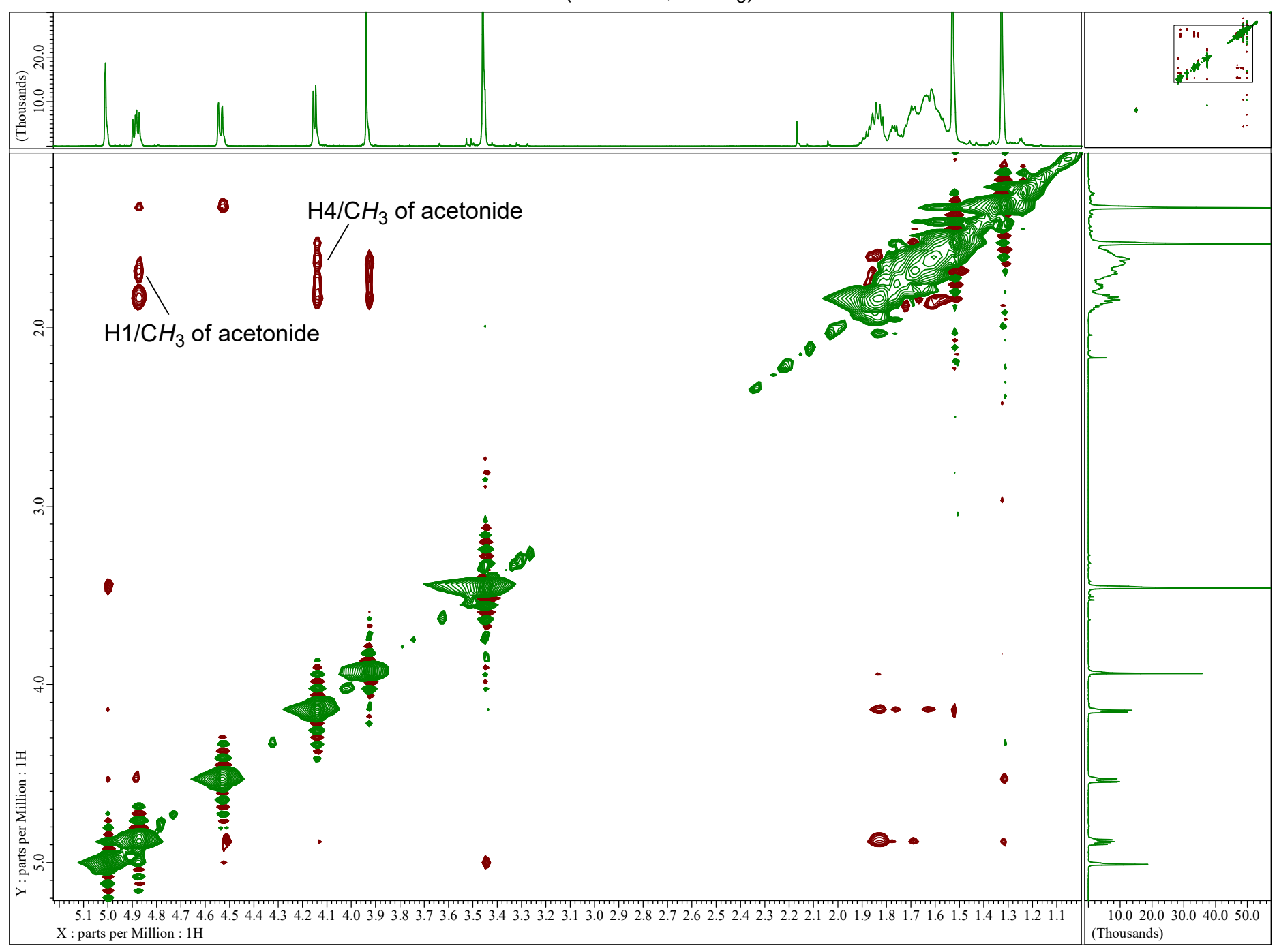


<smiles>COC(=O)C1OC(C)(C)O[C@H]1C1(C(C#N)C#N)CCCCC1</smiles>

5fh

${ }^{1} \mathrm{H}$ NMR (400 MHz, $\mathrm{CDCl}_{3}$ )

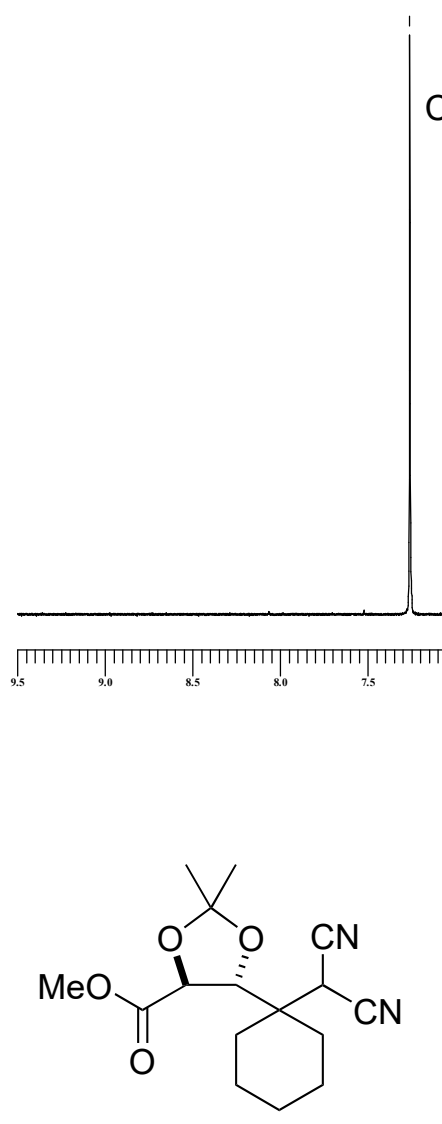

5fh

${ }^{13} \mathrm{C}\left\{{ }^{1} \mathrm{H}\right\}$ NMR $\left(100 \mathrm{MHz}, \mathrm{CDCl}_{3}\right)$

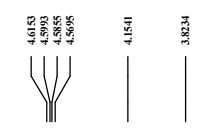

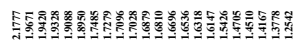

ㄴKWWWHIHI

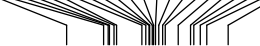
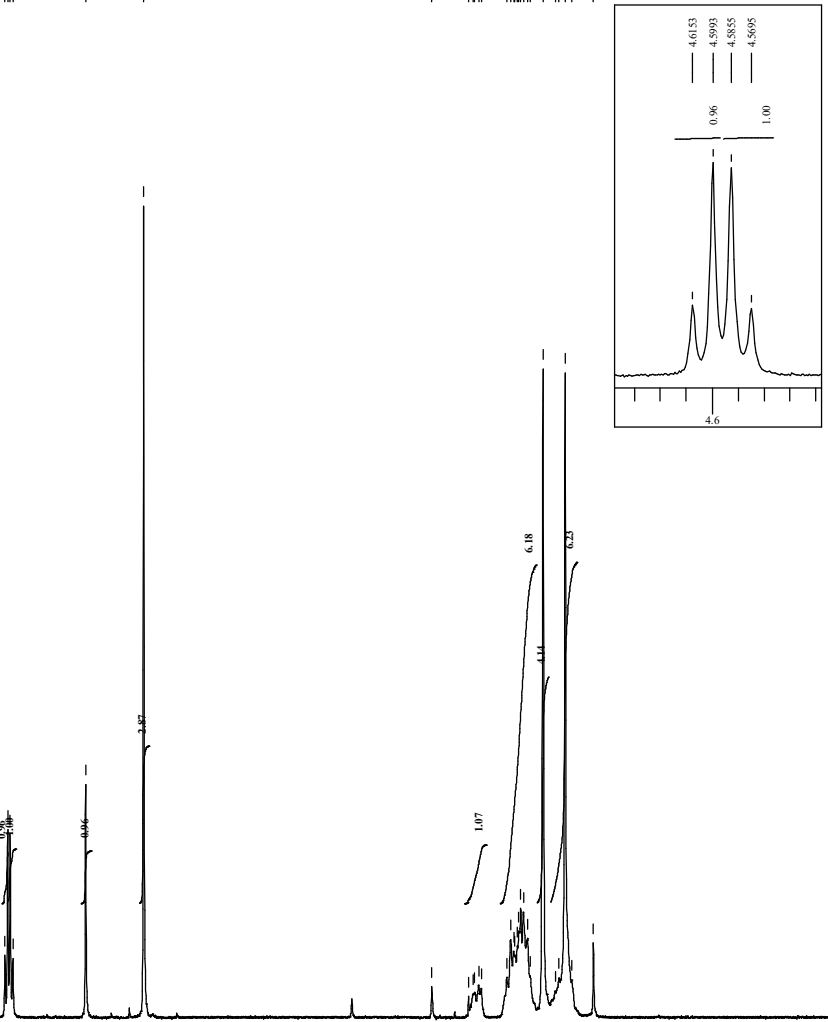

$\mathrm{HCl}_{3}$
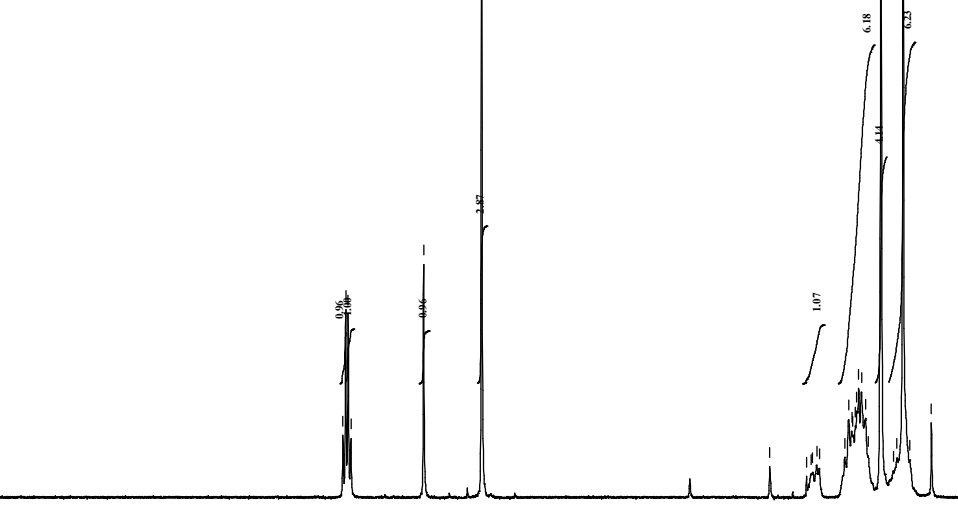


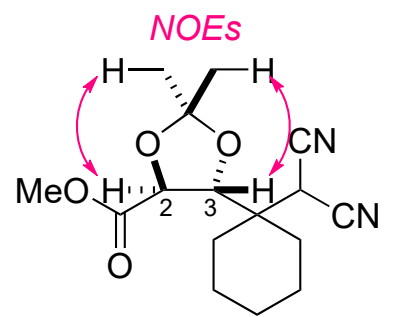

5 fh

NOESY $\left(400 \mathrm{MHz}, \mathrm{CDCl}_{3}\right)$

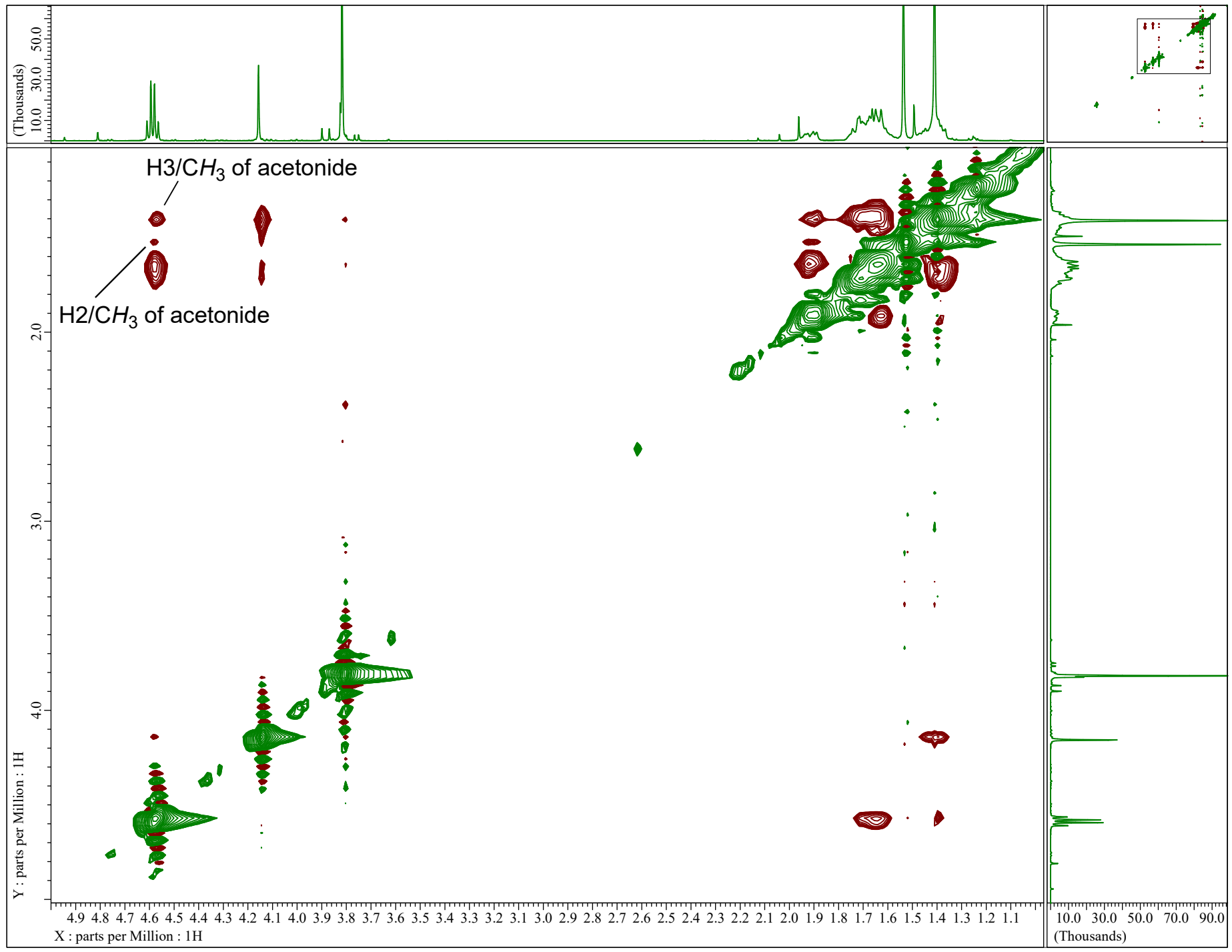




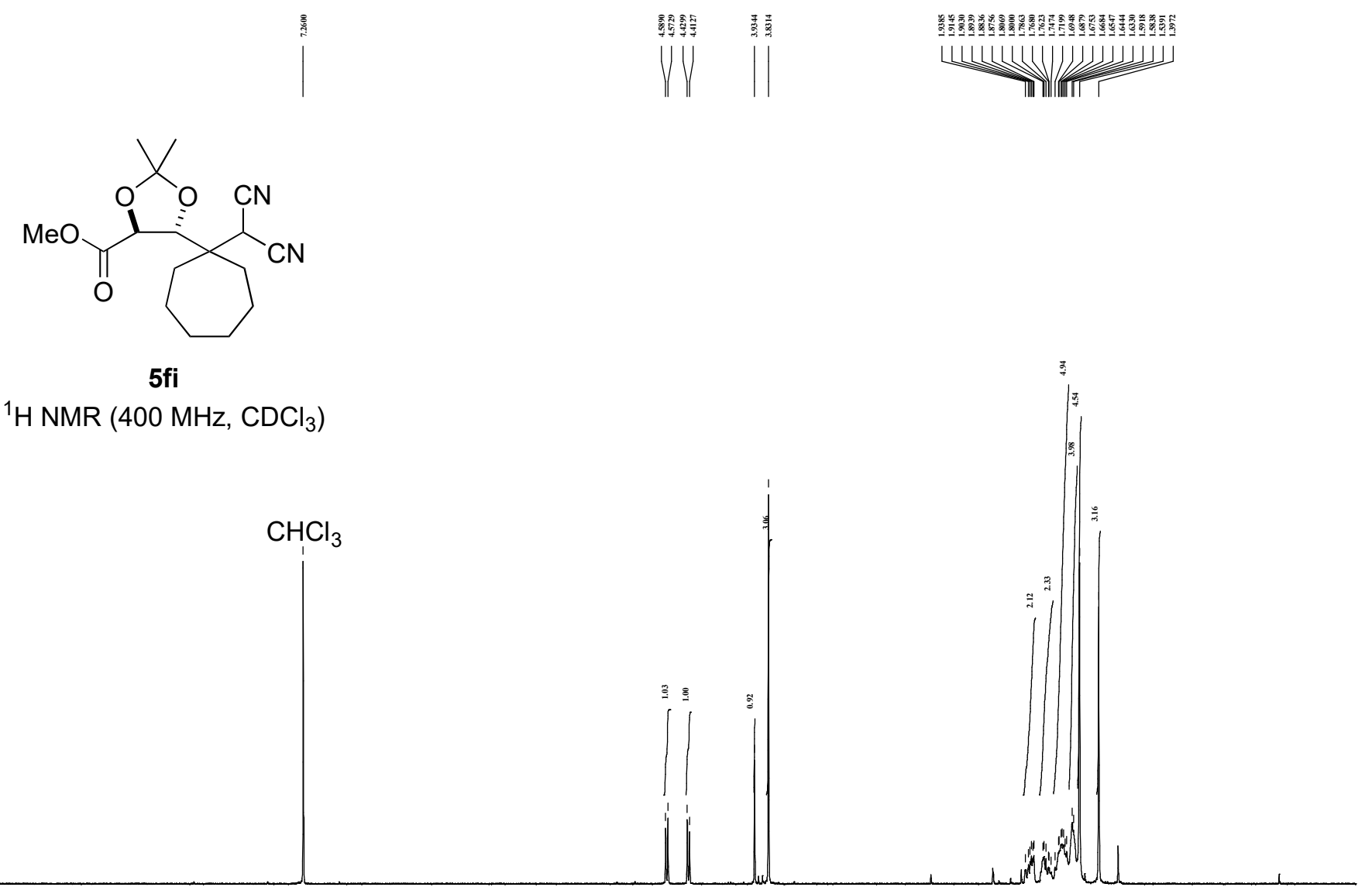

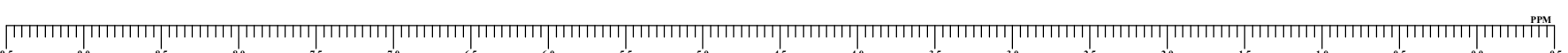

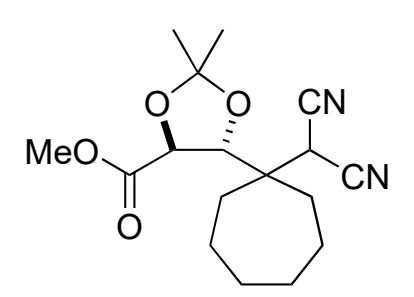

$5 \mathrm{fi}$

${ }^{13} \mathrm{C}\left\{{ }^{1} \mathrm{H}\right\} \operatorname{NMR}\left(100 \mathrm{MHz}, \mathrm{CDCl}_{3}\right)$

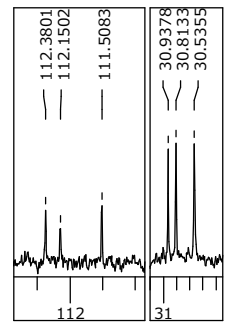

$\mathrm{CDCl}_{3}$

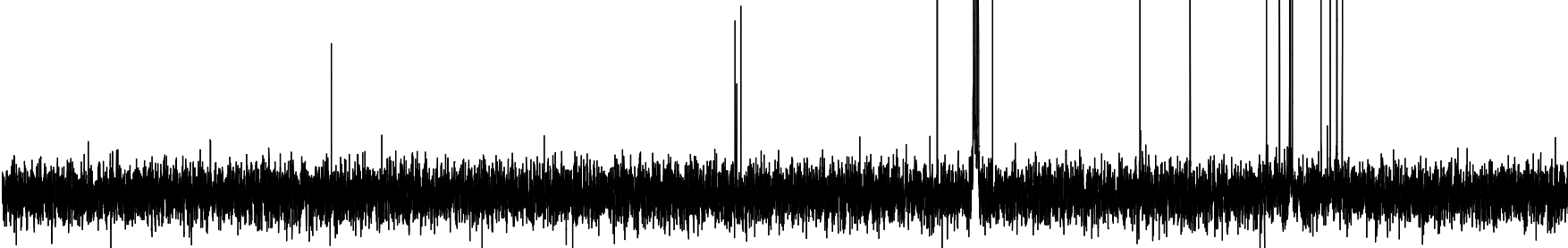

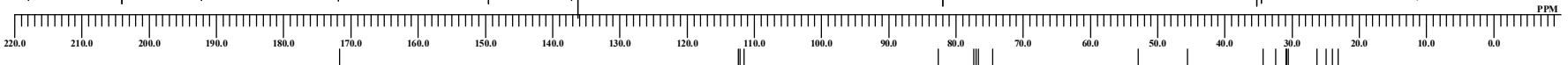

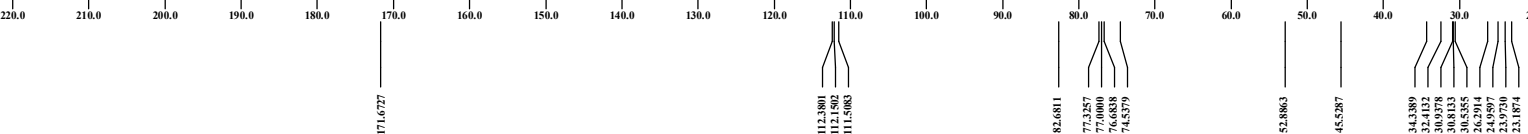




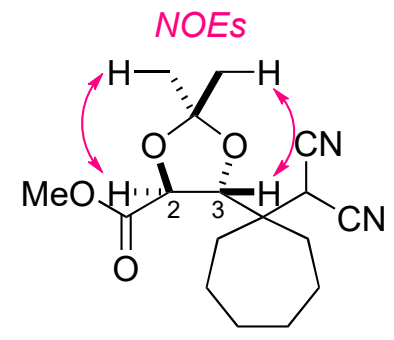

$5 \mathrm{fi}$

NOESY $\left(400 \mathrm{MHz}, \mathrm{CDCl}_{3}\right)$

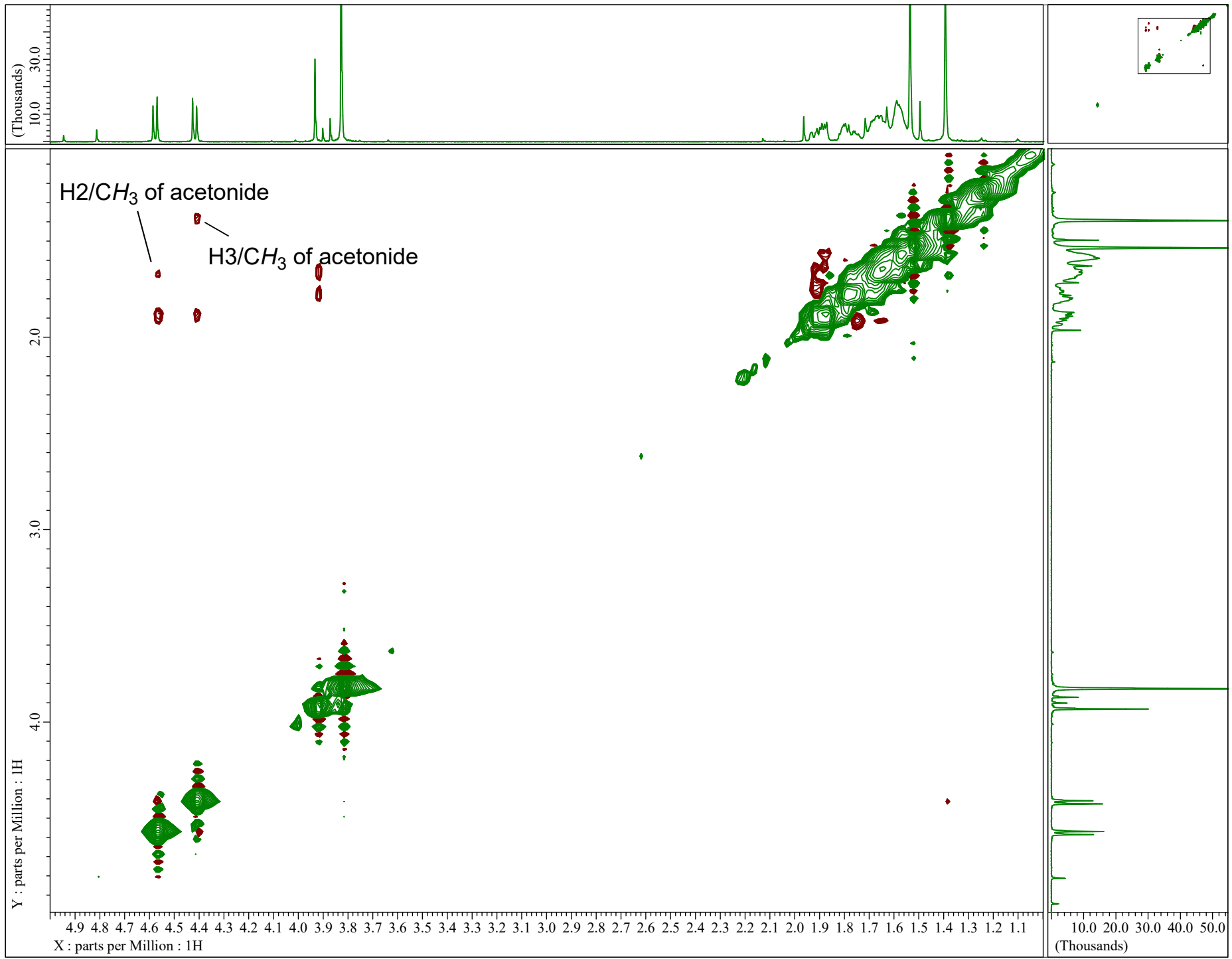


<smiles>CC12CC(CC(C3(C(C#N)C(C#N)C#N)CCCCC3)O1)O2</smiles>

5gh

${ }^{1} \mathrm{H}$ NMR $\left(400 \mathrm{MHz}, \mathrm{CDCl}_{3}\right)$

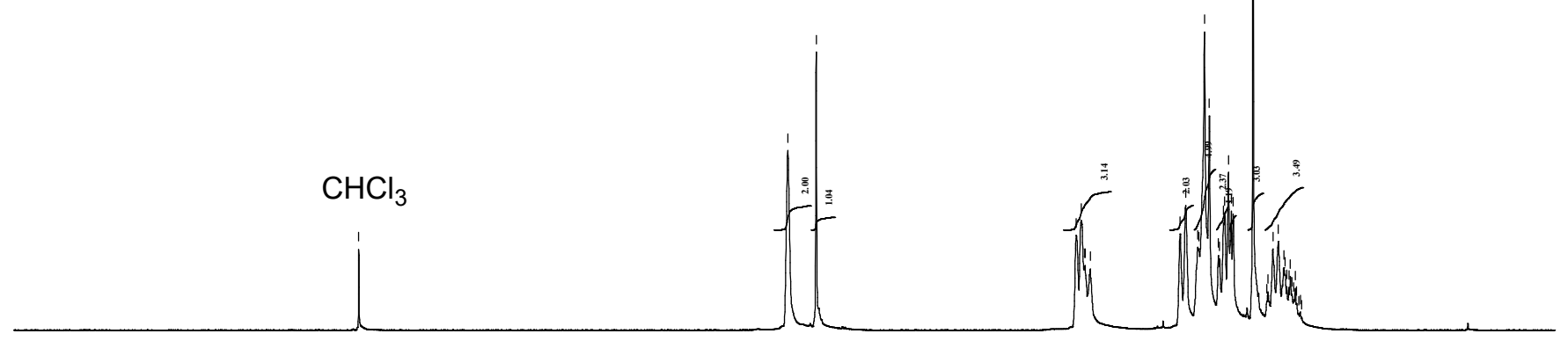

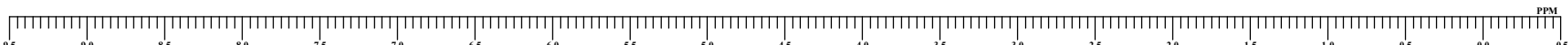
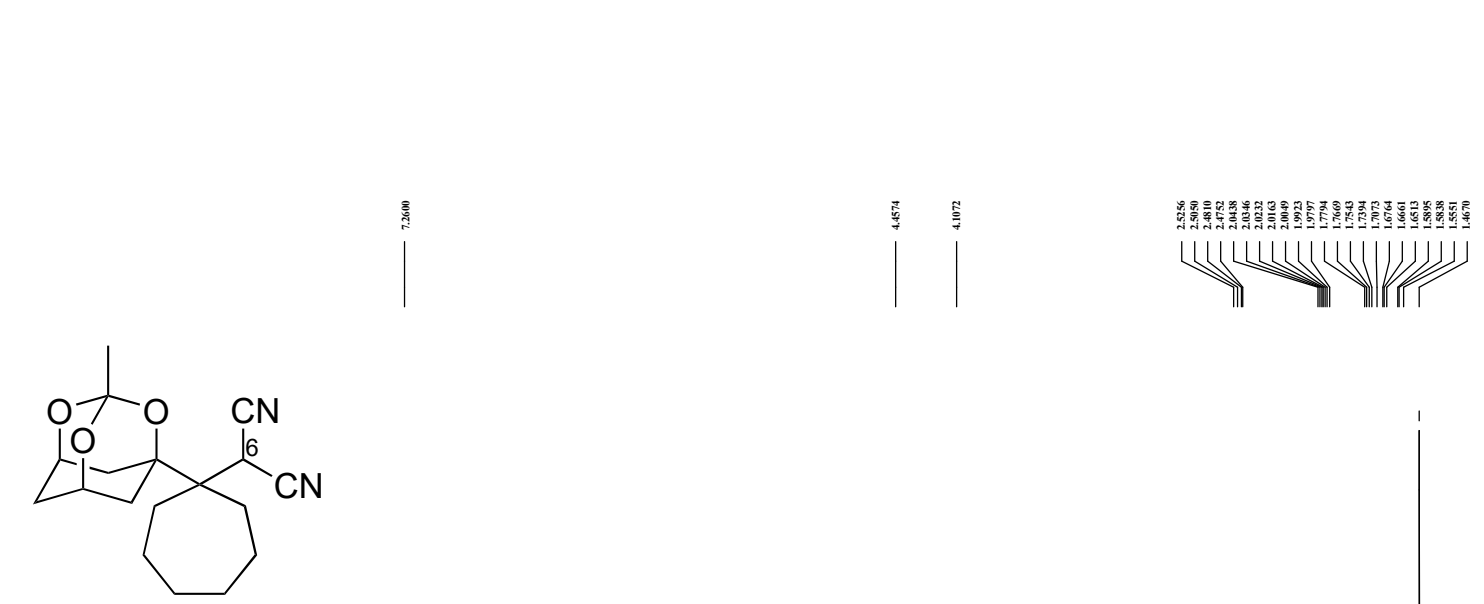

5 gi

${ }^{1} \mathrm{H}$ NMR $\left(400 \mathrm{MHz}, \mathrm{CDCl}_{3}\right)$

$\mathrm{CHCl}_{3}$
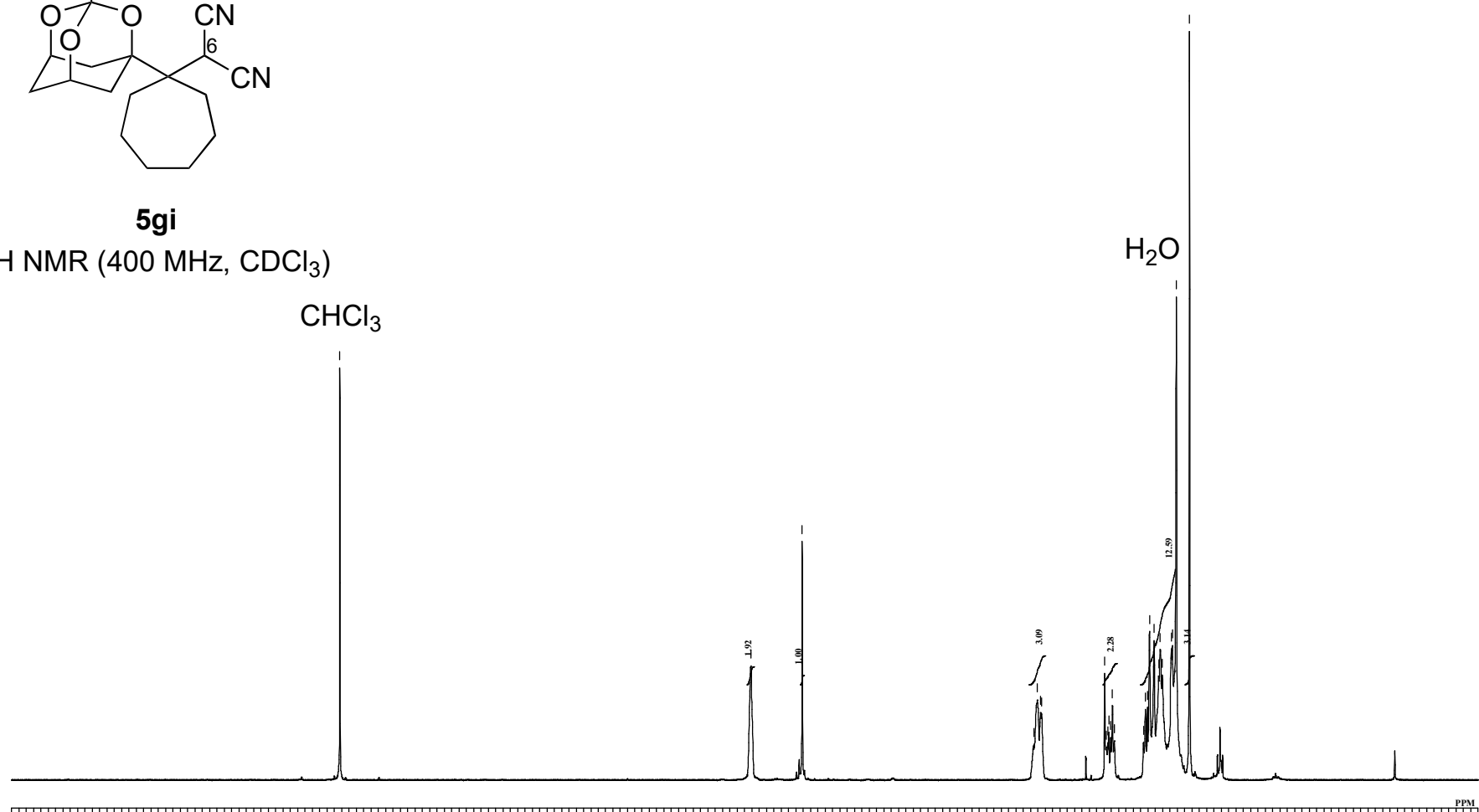

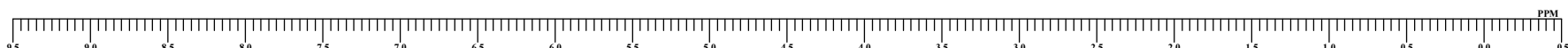




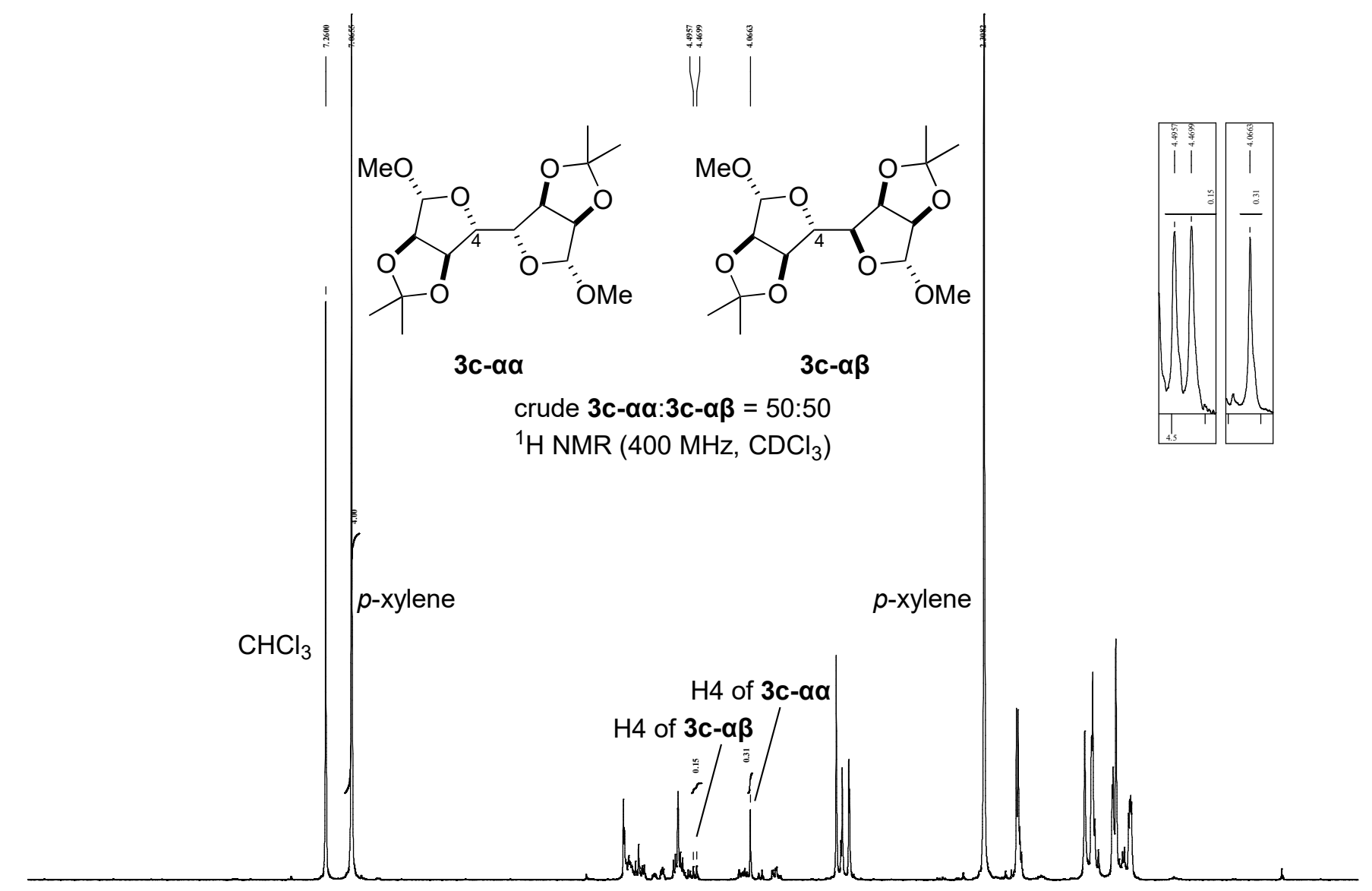

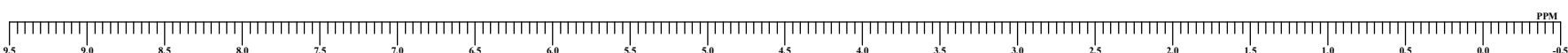
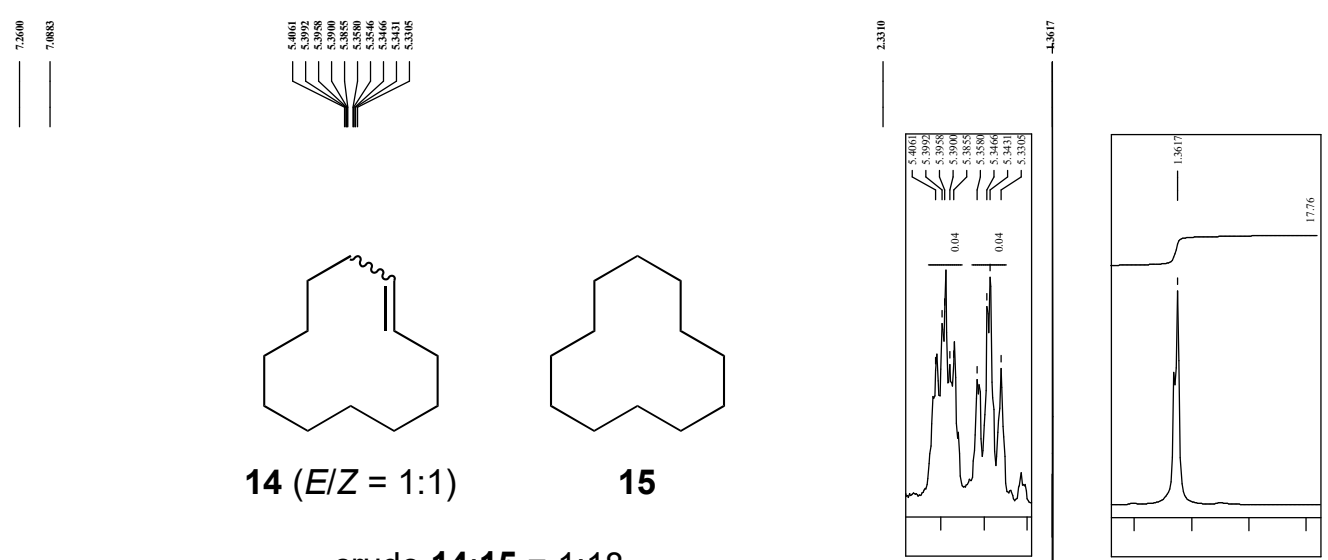

crude 14:15 $=1: 18$

${ }^{1} \mathrm{H}$ NMR $\left(400 \mathrm{MHz}^{\mathrm{CDCl}} 3\right)$
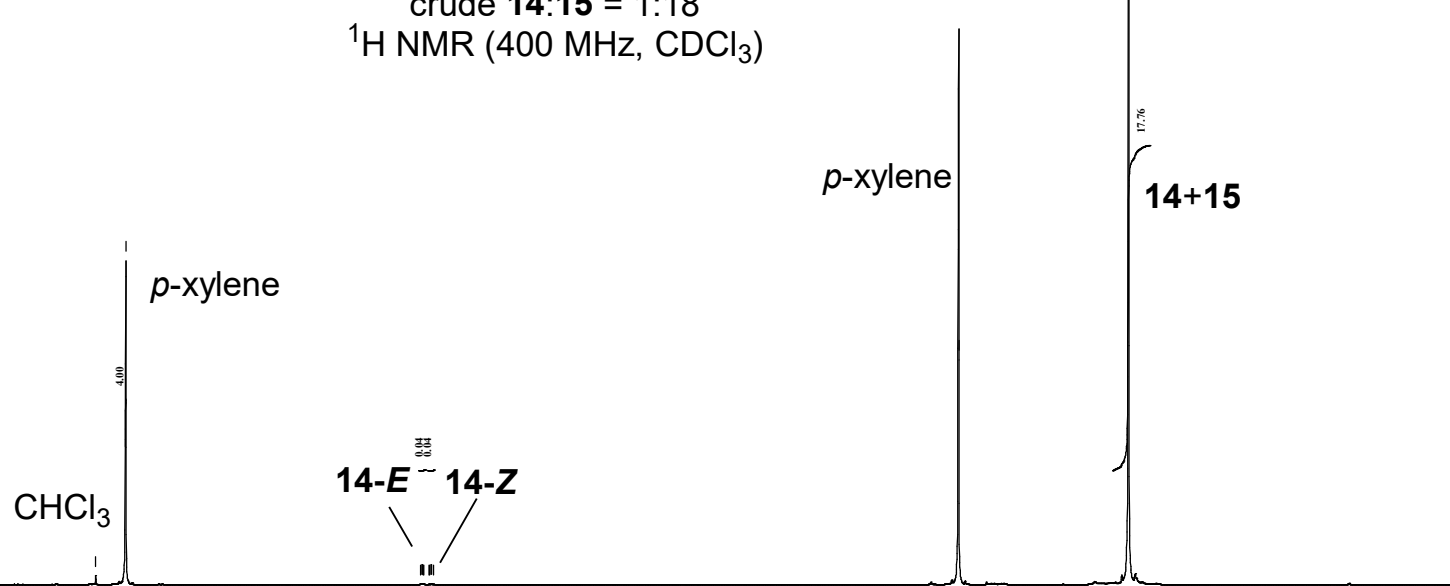

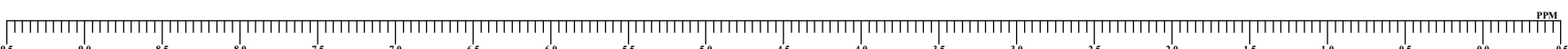




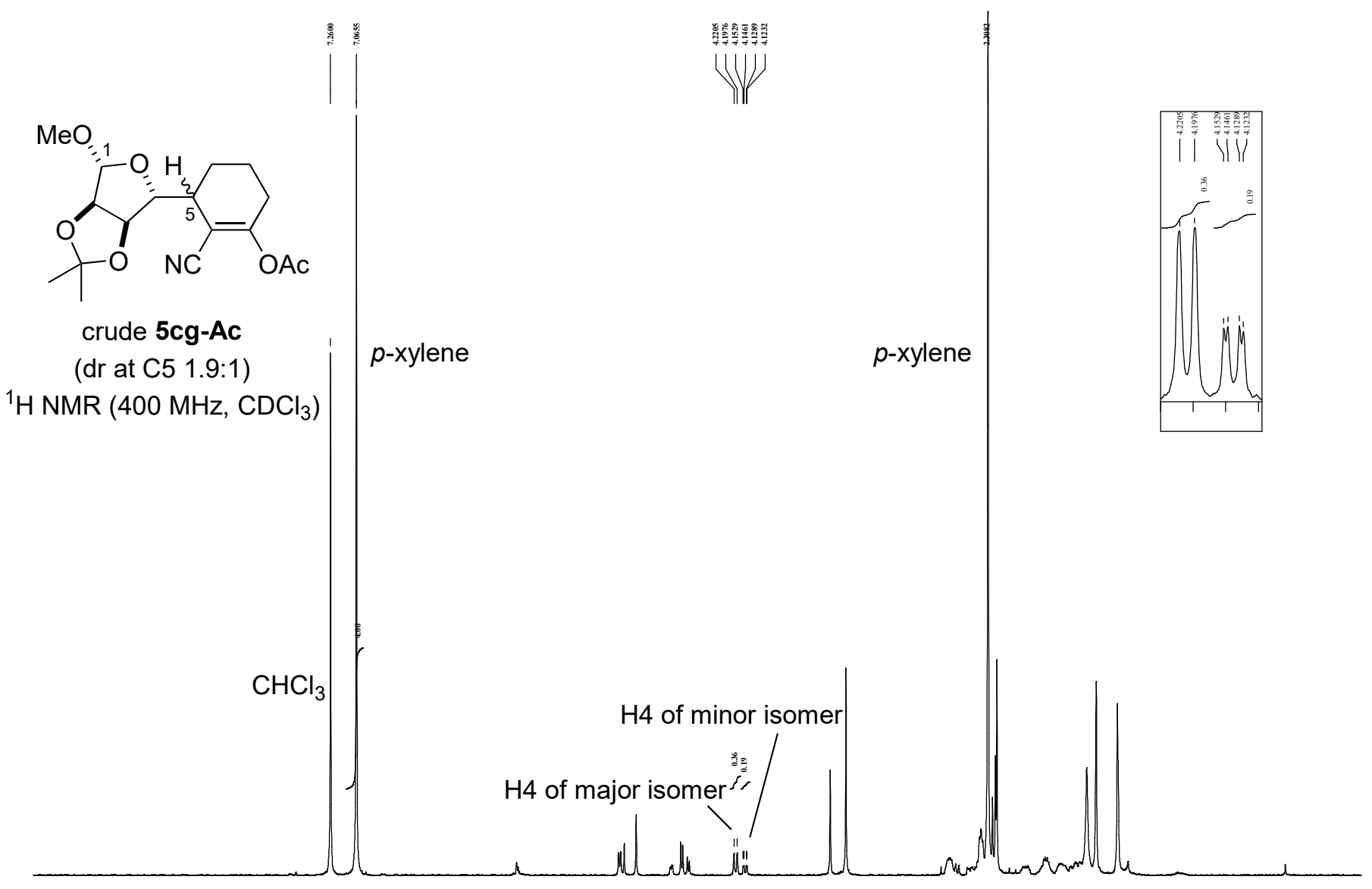

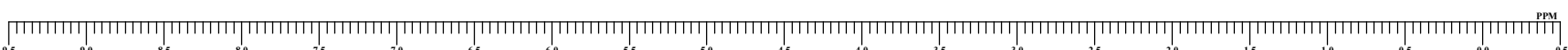

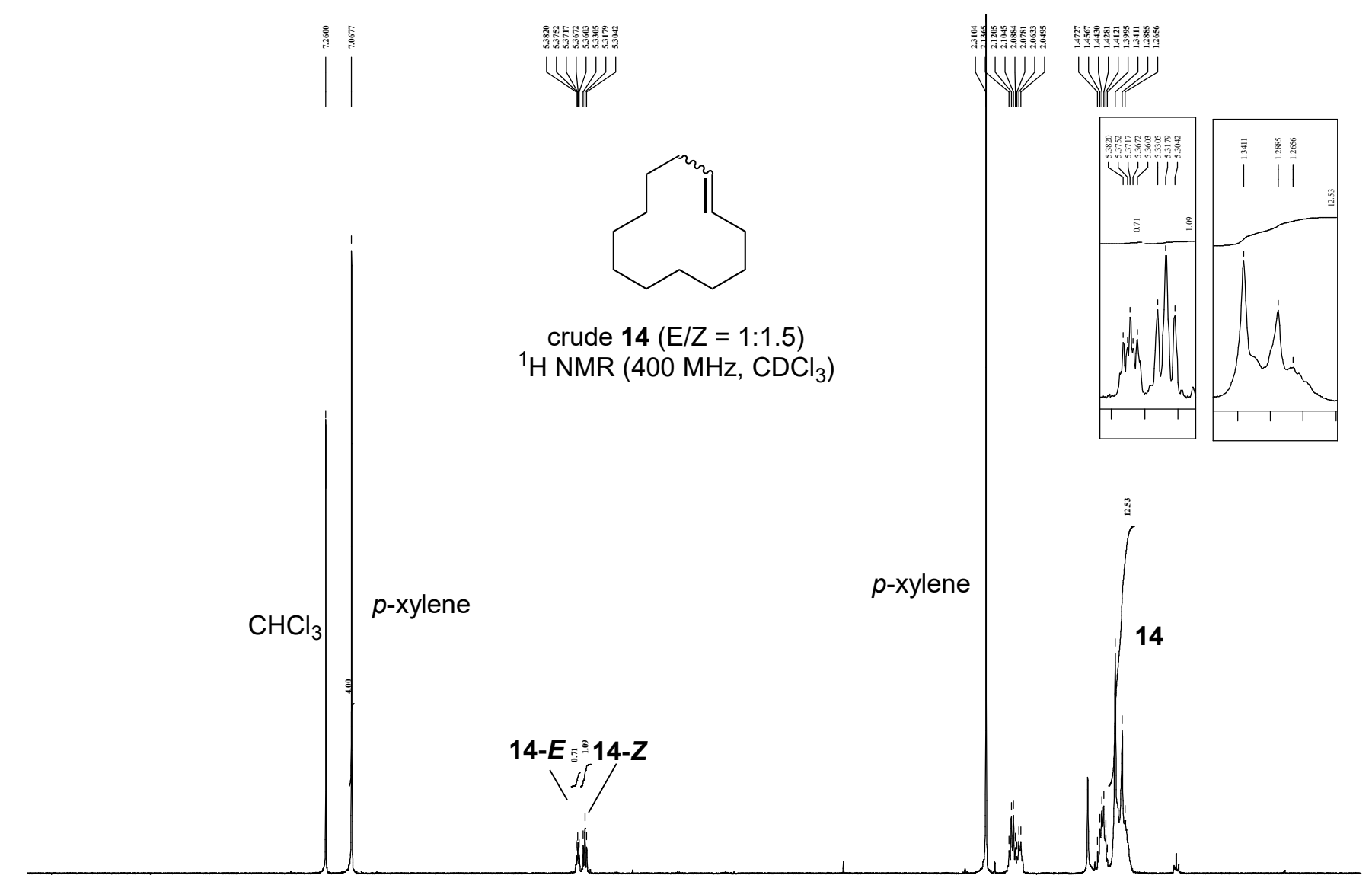

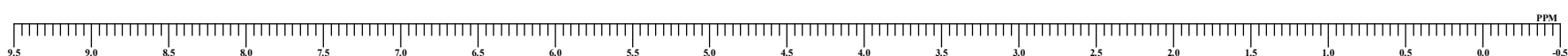

